Draft version May 30, 2018

Typeset using LATEX default style in AASTeX61

\title{
DEEP HUBBLE SPACE TELESCOPE IMAGING OF GLOBULAR CLUSTERS TOWARDS THE GALACTIC BULGE: OBSERVATIONS, DATA REDUCTION, AND COLOR-MAGNITUDE DIAGRAMS*
}

\author{
Roger E. Cohen, ${ }^{1,2}$ Francesco Mauro,,${ }^{2,3,4}$ Javier Alonso-García, ${ }^{5,3}$ Maren Hempel, ${ }^{6}$ Ata Sarajedini, ${ }^{7,8}$ \\ Antonio J. Ordoñez, ${ }^{9}$ Douglas Geisler, ${ }^{2}$ And Jason S. Kalirai ${ }^{1}$
}

\footnotetext{
${ }^{1}$ Space Telescope Science Institute, 3700 San Martin Drive, Baltimore, MD 21218, USA

${ }^{2}$ Departamento de Astronomía, Universidad de Concepción, Casilla 160-C, Concepción, Chile

${ }^{3}$ Millenium Institute of Astrophysics, Av. Vicuña Mackenna 4860, 7820436 Macul, Santiago, Chile

${ }^{4}$ Instituto de Astronomía, Universidad Católica del Norte, Av. Angamos 0610, Casilla 1280, Antofagasta, Chile

${ }^{5}$ Unidad de Astronomía, Fac. Cs. Básicas, Universidad de Antofagasta, Avda. U. de Antofagasta 02800, Antofagasta, Chile

${ }^{6}$ Instituto de Astrofísica, Facultad de Física, Pontificia Universidad Católica de Chile, Av. Vicuña Mackenna 4860, 782-0436 Macul, Santiago, Chile

${ }^{7}$ Dept. of Astronomy, University of Florida, 211 Bryant Space Sciences Center, Gainesville, FL 32611, USA

${ }^{8}$ Department of Physics, Florida Atlantic University, 777 Glades Road, Boca Raton, FL 33431, USA

${ }^{9}$ Insight Data Science Fellow
}

\begin{abstract}
The Galactic globular clusters (GGCs) located towards the Galactic bulge have generally been excluded from largescale photometric GGC surveys due to severe total and differential extinction. Here, we present an overview of a Hubble Space Telescope (HST) program designed to obtain deep, high spatial resolution multiband imaging of 16 poorly studied GGCs located towards the inner Galactic bulge and disk. In this first paper of a series resulting from these observations, we give an overview of target cluster selection, observations and data reduction procedures for optimizing the resulting photometric catalogs. Artificial star tests are used to compare the respective advantages of different data reduction strategies in terms of photometric and astrometric precision and photometric incompleteness. We present the resulting color-magnitude diagrams (CMDs) of all target clusters in several color-magnitude planes, along with CMDs of comparison fields from parallel observations. For each target cluster, we summarize existing studies, and discuss their CMDs qualitatively in the context of these results.
\end{abstract}

Corresponding author: Roger E. Cohen

rcohen@stsci.edu

* Based on observations made with the NASA/ESA Hubble Space Telescope, obtained at the Space Telescope Science Institute, which is operated by the Association of Universities for Research in Astronomy, Inc., under NASA contract NAS 5-26555. These observations are associated with program GO-14074 


\section{INTRODUCTION}

\subsection{The Power of a Self-consistent Globular Cluster Sample}

Galactic globular clusters (GGCs) carry the imprint of the early formation history of the Milky Way. Therefore, by studying GGCs as an ensemble as well as individually, they serve as powerful probes of Galactic dynamical and chemical evolution. Deep space-based photometric surveys have the power to yield heretofore unprecedented results in these arenas, as exemplified by the HST snapshot survey performed with the Wide Field Planetary Camera 2 (WFPC2) (Piotto et al. 2002), the Advanced Camera for Surveys (ACS) GGC Treasury Survey (Sarajedini et al. 2007), and the Ultraviolet Legacy Survey of GGCs (Piotto et al. 2015). The scientific output facilitated by such massive, self-consistent datasets is difficult to overstate, so as an example we focus on the ACS GGC Treasury Survey: By providing homogenous, deep photometry (SNR 10 at $\sim 0.2 M_{\odot}$ ) for 65 GGCs, this survey has yielded a plethora of self-consistent results covering a huge breadth of topics. These include cluster distances and reddenings (Dotter et al. 2010; Cohen \& Sarajedini 2012), both relative (Marín-Franch et al. 2009) and absolute ages (Dotter et al. 2010; VandenBerg et al. 2013), luminosity and mass functions (Paust et al. 2010), structural parameters and mass segregation (Goldsbury et al. 2010, 2013) and binary fractions (Milone et al. 2012). The impact of these studies is due largely to the self-consistency of the observation and data reduction strategy, allowing direct comparisons across the largest sample to date. However, such datasets inevitably have the added benefit of allowing in-depth analysis of individual cases of particular interest (e.g. Milone et al. 2008; Siegel et al. 2011). Furthermore, by obtaining high-quality imaging of many previously unstudied targets, their legacy value may be exploited in complimentary future campaigns which yield both a time baseline for dynamical analyses (e.g. Anderson \& van der Marel 2010; Samra et al. 2012; Bellini et al. 2014; Watkins et al. 2015a,b) as well as a broadened color baseline via imaging in complementary bandpasses (e.g. Dotter et al. 2015; Milone et al. 2015; Massari et al. 2016; Simunovic \& Puzia 2016; Wagner-Kaiser et al. 2016)

GGCs in the direction of the Galactic bulge have been almost entirely omitted from such large scale studies due to severe total and differential extinction. For example, 24 of the 26 GGCs within $2 \mathrm{kpc}$ of the Galactic center according to the catalog of Harris (1996, 2010 edition, hereafter H96) lack a self-consistent age estimate, and nearly one third of them $(8 / 26)$ lack any spectroscopic metallicity value from observations of individual stars ${ }^{1}$. Here, we present a set of observations which build on existing Treasury programs, allowing the GGCs located towards the bulge to be studied with the same level of scrutiny as the remainder of the GGC population.

\subsection{The Globular Clusters Towards the Galactic Bulge}

There are already tantalizing hints that the GGCs of the bulge hold valuable keys to Galactic astrophysics not found elsewhere. This is exemplified by the complex stellar system Terzan 5, which was not recognized as being significantly outside the traditional definition of Milky Way GGCs until the discovery of a double horizontal branch (HB) by Ferraro et al. (2009). This discovery was bolstered by subsequent studies revealing that Terzan 5 is one of the most massive GGCs (Lanzoni et al. 2010), and hosts stellar populations with a trimodal metallicity distribution spanning $\sim 1$ dex in $[\mathrm{Fe} / \mathrm{H}]$ (Origlia et al. 2013; Massari et al. 2014) and $\sim 7$ Gyr in age (Ferraro et al. 2016). Shortly thereafter, PSF photometry of $Z Y J H K_{S}$ imaging from the Vista Variables in the Via Lactea survey (VVV; Minniti et al. 2010) revealed evidence of similar, albeit less well separated, double HBs in two bulge GGCs which are also fairly massive and metal-rich, NGC 6440 and NGC 6569 (Mauro et al. 2012). However, in contrast to Terzan 5, a metallicity spread has been ruled out as a contributor to the HB morphology of these two clusters (Muñoz et al. 2017; Johnson et al. 2018) and in the case of NGC 6569, pulsational properties of its RR Lyrae variables also argue against helium enhancement (Kunder et al. 2015). More generally, the GGCs towards the Galactic center occupy unique areas of parameter space with respect to several key cluster parameters, including metallicity, HB morphology and concentration. For example, of the GGCs with $[\mathrm{Fe} / \mathrm{H}]<-0.5$, the majority are projected on the Galactic disk or bulge, including the only two (NGC 6528 and NGC 6553) that have approximately solar $[M / H]$ (e.g. Mauro et al. 2014, Dias et al. 2016, Tang et al. 2017). Furthermore, the incidence of candidate core-collapsed GGCs appears to increase substantially close to the Galactic center, and $17 / 28$ of them have $R_{G C} \leq 3 \mathrm{kpc}$ according to the H96 catalog.

Much of what we currently know about bulge GGCs is due to systematic efforts to characterize these clusters photometrically despite their higher total extinction and field star densities. Bulge GGCs have been specifically

\footnotetext{
1 This fraction drops to 6/26 if one considers the benchmark integrated light studies by Zinn \& West (1984) and Armandroff \& Zinn (1988), which included NGC 6333=M9 and Terzan 6 respectively.
} 
targeted using ground-based imaging at optical (e.g. Ortolani et al. 1997a, 1999a, 2006, see Bica et al. 2016 for a review) and near-infrared wavelengths (Valenti et al. 2010 and references therein ${ }^{2}$ ) as well as a handful of clusters imaged with the first generation of space-borne near-IR arrays (Ortolani et al. 2001a, 2007). More recently, widefield multi-wavelength photometric surveys such as the DECam Plane Survey (Schlafly et al. 2018) and VVV are providing additional information on clusters falling within their survey areas (e.g. Cohen at al. 2017). However, with a few recent exceptions (e.g. Cohen at al. 2014; LaGioia et al. 2014; Saracino et al. 2016; Kerber et al. 2018) existing imaging generally lacks adequate depth and/or spatial resolution to probe below the main sequence turnoff (MSTO) of bulge GGCs. Rather, luminous, evolved cluster red giant branch (RGB) and HB stars are used to estimate cluster distances, reddenings and metallicities, often via direct comparison with optically well-studied calibrating clusters (e.g. Valenti et al. 2004; Ferraro et al. 2006). However, the distance and metallicity scales of the calibrating clusters have since seen substantial improvement, at least in a relative sense (see e.g. figs. 18-19 of Cohen at al. 2015). Therefore, we aim to simultaneously harness the improvement in the parameters of optically well-studied GGCs together with deep, space-based imaging of the target bulge GGCs. By combining improved cluster photometric parameters with measurements of their ages and structural parameters, we gain two related angles of attack on fundamental questions of Galactic evolution.

One angle of attack is via the age-metallicity relation (AMR) of GGCs, which yields critical constraints on the assembly of the Milky Way: Recent evidence implies that metal-rich GGCs formed in the Galactic disk, whereas the more metal-poor halo GCs were accreted from satellite galaxies (e.g. Leaman et al. 2013). However, the formation history of the Galactic bulge and its GGCs remains an open question, as 24 of the 26 GGCs within 2 kpc of the Galactic center (H96) have been excluded from all recent systematic studies of GGC relative or absolute ages (De Angeli et al. 2005; Marín-Franch et al. 2009; Dotter et al. 2010; VandenBerg et al. 2013; Wagner-Kaiser et al. 2017; O'Malley et al. 2017). Age measurements of GGCs depend critically on their chemical abundances, but spectroscopic metallicity determinations with a precision of $<0.1$ dex for bulge GGCs are well within the reach of current observational capabilities via either high resolution optical spectroscopy (e.g. Barbuy et al. 2016; Muñoz et al. 2017) or near-IR single- (e.g. Valenti et al. 2015) or multi-object spectrographs (Tang et al. 2017; Fernández-Trincado et al. 2018). In this context, bulge GGC ages are the missing piece of the AMR puzzle, and the AMR of the innermost GGCs is a critical probe of bulge formation and evolution.

The second angle of attack makes use of structural and morphological parameters. The structural and morphological parameters of GGCs located towards the Galactic bulge are still based largely on either surface brightness profiles obtained from integrated light (Trager et al. 1995; McLaughlin \& van der Marel 2005), or star counts which rely on bright upper-RGB stars (Bonatto \& Bica 2008a). Both suffer from stochastic biases due to the low frequency of luminous cluster members as well as the effects of mass segregation within the GGCs (Noyola \& Gebhardt 2006; Goldsbury et al. 2013). These issues can be alleviated by the simultaneous use of stellar number densities in place of integrated light, together with wide field photometry, but thus far such investigations have targeted mostly halo GGCs (e.g. Miocchi et al. 2013) since extinction and high field star densities complicate analyses at low Galactic latitudes. For GGCs located towards the Galactic bulge, where line-of-sight extinction varies on spatial scales of arcseconds (e.g. Alonso-García et al. 2012; Cohen at al. 2014), the use of exclusively near-IR imaging is ideal. Particularly when widefield near-IR imaging extending beyond the cluster outskirts is available, as in the present case (Cohen at al. 2017, see Sect. 2.1), the marriage of such a catalog with high-spatial resolution near-IR imaging of cluster cores is necessary to circumvent strong total and/or variable extinction. In at least one case, application of this strategy has revealed structural parameters substantially different from previous values based on optical integrated light (Saracino et al. 2015).

Forthcoming papers in this series will present in-depth quantitative analyses of the aforementioned issues, while here we present an overview of our imaging campaign and data reduction strategies. The next section describes our target cluster selection and overall observation strategy. In Section 3, we describe our data processing, including PSF photometry, artificial star tests, and the resulting completeness limits. In Section 4, we present CMDs for each of our target clusters in several color-magnitude planes, discussing their features in the context of previous literature studies for each cluster. Our results are summarized in the final section.

\section{OBSERVATIONS}

2 http://www.bo.astro.it/ GC/ir_archive/Tab1_new.html 


\subsection{Target Cluster Selection}

Candidate target clusters were selected to meet several requirements: First, they have wide field near-infrared imaging from the VVV survey. This imaging is crucial to the construction of radial number density profiles extending over the entire radial extent of each cluster. Our catalogs of VVV PSF photometry carry the added benefit of providing a network of secondary astrometric standards much more numerous than either 2MASS or optical astrometric catalogs in heavily extincted regions. Our targets are therefore spatially restricted to the VVV bulge $(-10.0 \lesssim l \lesssim 10.5,-10.3 \lesssim b \lesssim 5.1)$ and disk $(294.7 \lesssim l \lesssim 350.0,-2.25 \lesssim b \lesssim 2.25)$ survey areas. In Fig. 1 , we show a map of the VVV bulge survey area in Galactic coordinates, noting that only one of our target clusters (FSR 1735) lies in the VVV disk, rather than bulge, survey area ${ }^{3}$. In Fig. 1, all GGCs have been color-coded by their metallicity from H96, and all of our targets are shown as filled circles, while the four GGCs (NGC 6441, NGC 6624, NGC 6637=M69, NGC 6656=M22) with deep archival Treasury and Legacy survey imaging (which include the same ACS/WFC F606W filter we employ) are shown as filled diamonds.

The second criterion imposed on our target list is that our target clusters are relatively poorly studied, and lack archival imaging of sufficient depth or spatial resolution to measure cluster ages (i. e. extending significantly faintward of the MSTO). Our results therefore include the first unambiguous detection of the MSTO for these GGCs in many cases $^{4}$. However, it bears mention that for the handful of GGCs in the VVV survey area with deep archival HST imaging not obtained as part of the Treasury Survey, shown as open diamonds in Fig. 1, age analyses are complicated by the heterogeneity of the available data. Archival imaging of these clusters was obtained through different programs employing various filter and instrument combinations, but also different photometric depths, hampering the use of relative CMD indices for age measurements (e.g. VandenBerg et al. 2013, and references therein), particularly for clusters which saturate only slightly brightward of the MSTO.

To arrive at the final target list, we decided to eliminate three additional clusters from our sample (2MASS GC02, Liller 1, UKS 1) which all have $E(B-V)>3$ according to the GGC catalog of H96. This level of interstellar extinction is prohibitive to obtaining sufficiently deep WFC3/IR imaging in one orbit, and also to obtaining ACS/WFC optical imaging below the MSTO in a small number of orbits. For this reason, we have also excluded several additional candidate GGCs (Bonatto et al. 2009; Minniti et al. 2011; Ortolani et al. 2012) pending independent constraints from ground-based observations employing either spectrocopy (Peñaloza et al. 2015), time series imaging of variable stars (Alonso-García et al. 2015), and/or high spatial resolution near-IR follow-up imaging (e.g. Saracino et al. 2015).

Our target clusters are listed in Table 1 along with their equatorial and Galactic coordinates and total exposure times for the primary field in each filter.

\subsection{Primary Fields}

\subsubsection{Observing Strategy}

Each primary field is observed for one orbit with ACS/WFC in $F 606 W$, and one orbit with WFC3/IR, splitting the orbit between $F 110 W$ and $F 160 W$. Despite large total and differential extinction towards many of our target clusters, there are three reasons why the ACS/WFC imaging provides a valuable complement to WFC3/IR:

1. Spatial resolution: Since WFC3/IR has $0.13^{\prime \prime} /$ pix, photometry of cluster cores is crowding-limited. The ability to leverage the increased spatial resolution of ACS/WFC (0.05"/pix) together with the WFC3/IR observations significantly enhances our ability to recover stars in the innermost regions of the target clusters, and improves overall positional accuracy (see Sect. 3.5).

2. Field of view: The ACS/WFC field of view $\left(202 \times 202^{\prime \prime}\right.$ per exposure $)$ is large enough compared to the WFC3/IR field of view $\left(123 \times 136^{\prime \prime}\right.$ per exposure $)$ that the WFC3/IR observations of all target clusters are colocated completely within the ACS/WFC field of view. This allows us to provide a complimentary bandpass of optical imaging for all relatively bright WFC3/IR sources. In addition, number density profiles covering the entire radial extent of target GGCs require a range of radial overlap between wide-field (typically shallower) imaging of the cluster outskirts and high-resolution imaging of the cluster cores, so the ACS/WFC observations give the option to choose increased radial coverage over increased photometric depth. Moreover, given the higher

\footnotetext{
3 The reclassification of the cluster FSR 1716 as a candidate globular cluster, located in the VVV disk survey area, was recently announced by Minniti et al. (2017).

${ }^{4}$ For the more crowded and/or extincted targets, the MSTO is often difficult to discern even from VVV PSF photometry of cluster cores because of a combination of lower spatial resolution $\left(0.339^{\prime \prime} / \mathrm{pix}\right)$ and lower $\mathrm{S} / \mathrm{N}$ relative to the color baseline than we obtain here.
} 


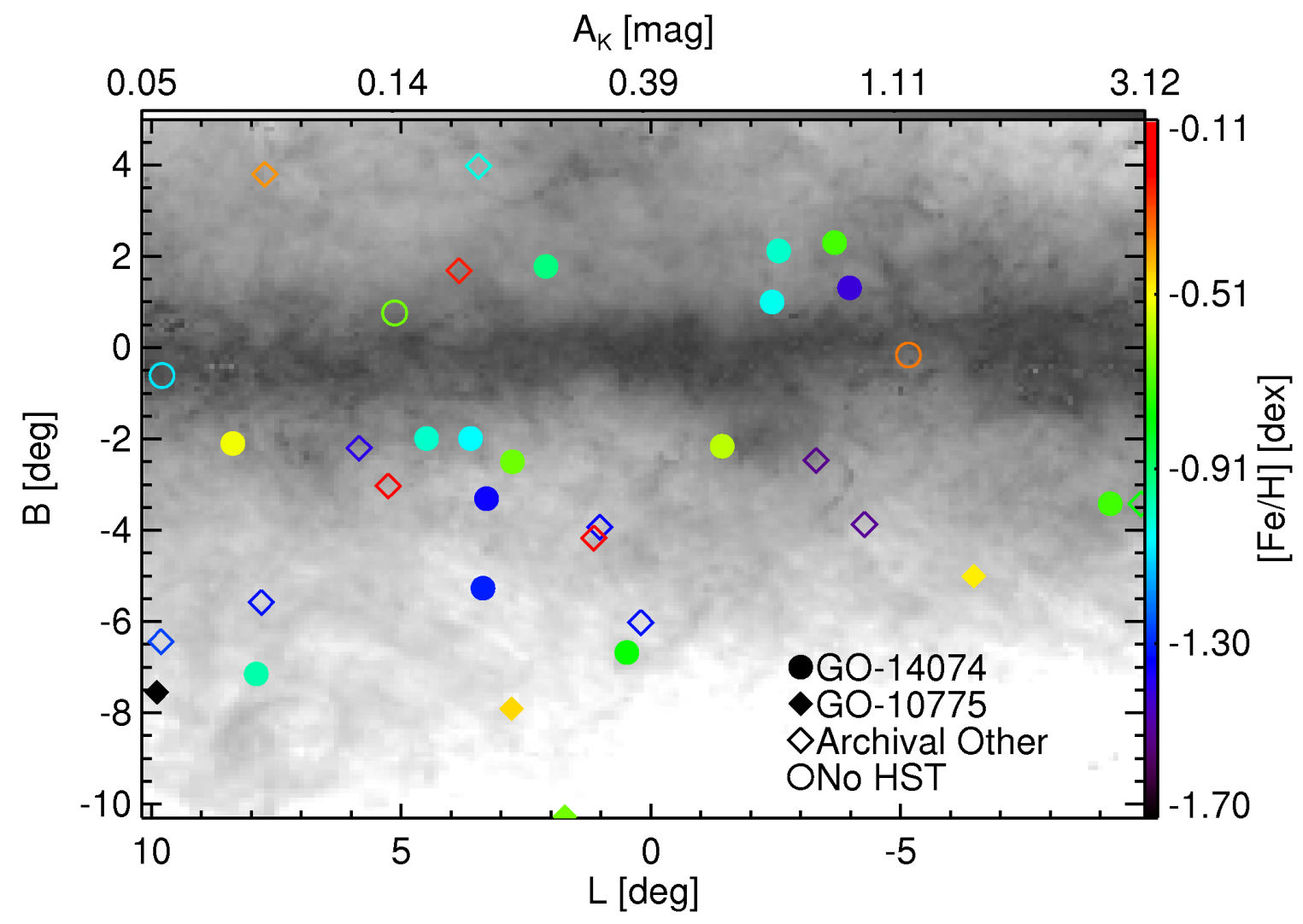

Figure 1. Location of all GGCs from the H96 catalog located within the VVV bulge survey area. Grey shading indicates the total $K_{S}$-band extinction from the map of Gonzalez et al. (2012), shown on a logarithmic greyscale (horizontal color bar). Clusters are color-coded by H96 $[\mathrm{Fe} / \mathrm{H}]$ (vertical color bar). Our target clusters are shown as filled circles, clusters with deep ACS/WFC photometry from GO-10775 are shown as filled diamonds, clusters with archival HST imaging from other programs are shown as open diamonds, and the three clusters with no HST imaging are shown as open circles. The target cluster FSR 1735 , at $l=-20.808, b=-1.854$, is not shown.

spatial resolution of ACS/WFC over WFC3/IR, the larger field of view also provides larger radial coverage of each cluster for future dynamical investigations which could utilize our ACS/WFC imaging as a first epoch to measure relative proper motions.

3. Color baseline: The precision of CMD-based analyses, including differential reddening corrections and age measurements, depends on the ratio of the observed color range to the photometric measurement uncertainty at a given CMD location. By complementing deep near-IR imaging with a bandpass of optical photometry, we can exploit smaller relative uncertainties in photometric measurements for the more optically luminous cluster members.

\subsubsection{Filters}

Our choice of filters for each instrument was made not only to maximize throughput, but also to complement existing imaging. This approach allows direct comparison to archival observations of both template GGCs as well as template bulge/disk fields in the native photometric system. We choose $F 606 \mathrm{~W}$ for ACS/WFC imaging, since it allows a broad color baseline when paired with near-IR filters from either HST or ground-based facilities. At the same time, direct comparisons can be made with the large set of GGCs observed in GO-10775 (Sarajedini et al. 2007) and additional archival imaging (e.g. Dotter et al. 2011; LaGioia et al. 2014) using, for example, $\Delta F 606 \mathrm{~W}$ as a distance-independent CMD-based metric. Similarly, for WFC3/IR observations, $F 110 W$ and $F 160 W$ not only yield the best compromise between color baseline and reddening insensitivity, but also allow a direct comparison to archival imaging of bulge and disk fields and template GGCs spanning a range of metallicities (e.g. Brown et al. 2010; Ross et al. 2014; Correnti et al. 2016). 
Table 1. Target Clusters

\begin{tabular}{|c|c|c|c|c|c|c|c|c|}
\hline \multirow[t]{2}{*}{ Cluster } & \multirow[t]{2}{*}{ Other Name } & $\mathrm{RA}(\mathrm{J} 2000)^{\mathrm{a}}$ & $\operatorname{Dec}(J 2000)^{\mathrm{a}}$ & $\mathrm{l}^{\mathrm{a}}$ & $b^{a}$ & $\mathrm{t}(F 606 W)$ & $\mathrm{t}(F 110 W)$ & $\mathrm{t}(F 160 W)$ \\
\hline & & hh:mm:ss.s & -dd:mm:ss.s & $\circ$ & $\circ$ & $\mathrm{s}$ & $\mathrm{s}$ & $\mathrm{s}$ \\
\hline ВH261 & ESO $456-78$ & $18: 14.1$ & $-28: 37$ & 3.37 & -5.27 & 1990 & 1246.154 & 1271.155 \\
\hline Djorg 2 & ESO 456-38 & 18:01:49.1 & $-27: 49: 33.0$ & 2.7636 & -2.5085 & 2000 & 1246.154 & 1271.155 \\
\hline FSR 1735 & 2MASS GC-03 & $16: 52: 12$ & $-47: 03.3$ & -20.808 & -1.854 & 2152 & 1296.155 & 1346.156 \\
\hline HP 1 & ESO 455-11 & $17: 31: 05.2$ & $-29: 58: 54.0$ & -2.5748 & 2.1150 & 2020 & 1246.154 & 1271.155 \\
\hline NGC 6540 & Djorg 3 & 18:06:08.6 & $-27: 45: 55.0$ & 3.2851 & -3.3130 & 1990 & 1246.154 & 1271.155 \\
\hline NGC 6569 & ESO 456-77 & $18: 13: 38.88$ & $-31: 49: 35.2$ & 0.4814 & -6.6809 & 2024 & 1246.154 & 1271.155 \\
\hline NGC 6638 & Gcl 95 & $18: 30: 56.25$ & $-25: 29: 47.1$ & -7.8977 & -7.1530 & 1990 & 1246.154 & 1271.155 \\
\hline Palomar 6 & ESO 520-21 & $17: 43: 42.2$ & $-26: 13: 21.0$ & 2.0921 & 1.7801 & 2020 & 1246.154 & 1271.155 \\
\hline Terzan 1 & ESO 455-23 & $17: 35: 47.8$ & $-30: 28: 11.0$ & -2.4311 & 0.9958 & 2043 & 1246.154 & 1271.155 \\
\hline Terzan 2 & ESO 454-29 & $17: 27: 33.2$ & $-30: 48: 07.8$ & -3.6802 & 2.2978 & 2036 & 1246.154 & 1271.155 \\
\hline Terzan 4 & Gcl 66.1 & $17: 30: 38.9$ & $-31: 35: 44.0$ & -3.9762 & 1.3080 & 2036 & 1246.154 & 1271.155 \\
\hline Terzan 6 & ESO 455-49 & $17: 50: 46.4$ & $-31: 16: 31.0$ & -1.4286 & -2.1618 & 2036 & 1246.154 & 1271.155 \\
\hline Terzan 9 & Gcl 80.1 & 18:01:38.8 & $-26: 50: 23.0$ & 3.6031 & -1.9888 & 2012 & 1246.154 & 1271.155 \\
\hline Terzan 10 & ESO 521-16 & $18: 02: 57.4$ & $-26: 04: 00.0$ & 4.4207 & -1.8629 & 2022 & 1246.154 & 1271.155 \\
\hline Terzan 12 & ESO 522-1 & $18: 12: 15.8$ & $-22: 44: 31.0$ & 8.3581 & -2.1008 & 2010 & 1246.154 & 1271.155 \\
\hline Ton 2 & ESO 333-16 & $17: 36: 11$ & $-38: 33.0$ & 9.201 & -3.421 & 2020 & 1271.155 & 1271.155 \\
\hline
\end{tabular}

$a_{\text {Literature values for cluster centers are taken from the SIMBAD database. }}$

\subsubsection{Exposure Sequences}

The exposure sequence for each instrument was designed based on proven strategies from previous imaging campaigns to obtain the best compromise between photometric depth and dynamic range. The ACS/WFC observations were obtained using the strategy from GO-10775, in which a single orbit is used to obtain one short exposure and four dithered long exposures in $F 606 \mathrm{~W}$. The dither step is large enough to cover the chip gap and provide contiguous imaging over the entire field of view, and the short exposure times are varied between $10 \mathrm{~s}$ and $60 \mathrm{~s}$ based on both orbital visibility constraints and total optical extinction towards each cluster. This allows the cluster HB to remain unsaturated in all cases, while obtaining maximum $\mathrm{S} / \mathrm{N}$ within a single orbit for heavily extincted clusters with fainter apparent HB magnitudes in F606W.

For WFC3/IR primary observations, we use a strategy similar to GO-11664, with the goal of obtaining the deepest possible photometry within one orbit without saturating the cluster HBs. Therefore, we interlace $F 110 W$ and $F 160 W$ exposures using STEP100 sequences of varying length with NSAMP ranging from 5 to 12 . Observations are obtained at three dither points in both filters to mitigate cosmetic defects in the WFC3/IR array.

\subsection{Parallel Fields}

Imaging of the primary field of each target cluster is supplemented with WFC3/IR $F 110 W$ and $F 160 W$ imaging of a parallel field centered $\sim 6$ arcmin from the center of the primary field. As these exposures are obtained during the dithered ACS/WFC observations of the primary field, the need to read out and dither ACS/WFC limited the available WFC3/IR observing sequences. As a result, for each parallel field we employed one STEP100 NSAMP=9 and one STEP100 NSAMP=12 WFC3/IR sequence per filter, for a total per-filter exposure time of $898.463 \mathrm{~s}$. In this way, we are able to attain a photometric depth comparable to the primary field WFC3/IR observations, noting that the shorter exposure times are counterbalanced to some extent by the reduced impact of crowding in the parallel fields 


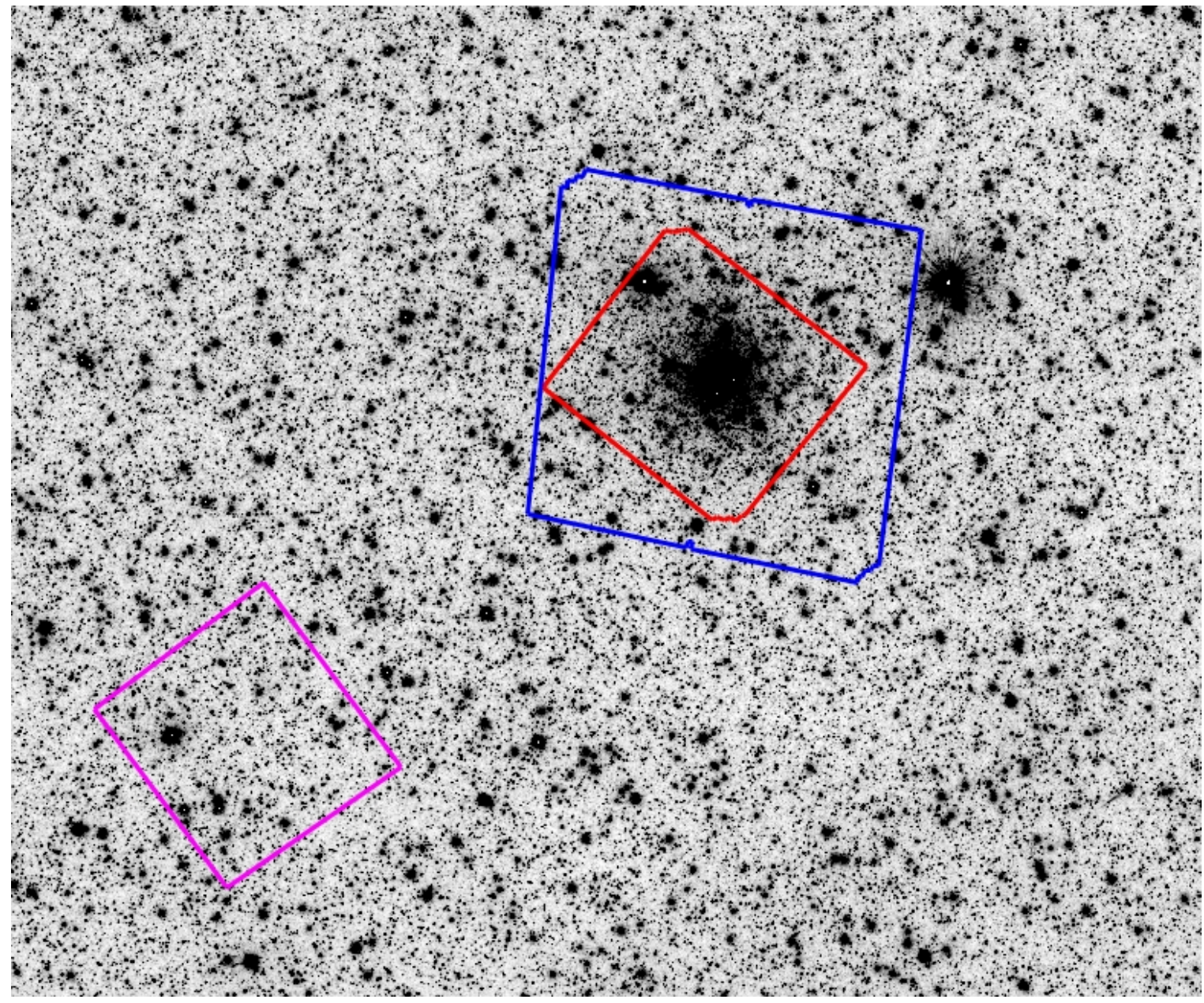

Figure 2. A $12^{\prime} \times 10^{\prime}$ portion of a VVV $K_{S}$-band image illustrating the relative orientations of the HST GO-14074 fields for the example of Terzan 1. The primary fields are shown for ACS/WFC (blue) and WFC3/IR (red), and the WFC3/IR parallel field is shown in magenta. North is up and east is to the left, and this VVV image has $0.339^{\prime \prime} /$ pix and was observed under seeing of $\sim 0.72^{\prime \prime}$.

far from the cluster centers. We placed no requirement on the relative orientation between the primary and parallel fields to optimize schedulability of the observations, so the angle between primary and parallel fields varies from cluster to cluster. However, to illustrate their relative sizes and orientations, the location of the parallel field relative to the primary fields is shown for the example case of Terzan 1 in Fig. 2.

\section{PHOTOMETRY}

We perform preprocessing and PSF photometry using the latest version of Dolphot 2.0 (Dolphin 2000). Dolphot provides preprocessing and photometry modules tailored to each HST camera, including pre-computed PSFs for each filter of each instrument and, crucially in our case, the ability to photometer images from multiple cameras simultaneously. A description of Dolphot, as well as recommended photometry parameters for each instrument (hereafter referred to as "default" values), can be found in the manuals available at the Dolphot website ${ }^{5}$.

\subsection{Preprocessing and Image Alignment}

The best multi-band ( $F 606 W, F 110 W, F 160 W$ ) photometry resulted from a two-tier data reduction strategy. First, images from each camera (ACS/WFC or WFC3/IR) are preprocessed separately, largely according to prescriptions in the Dolphot manual. Briefly, pixels flagged in the data quality images are masked from any further incorporation,

5 http://americano.dolphinsim.com/dolphot/ 
and each science image is split into a single extension fits file per chip (one for WFC3/IR, one for each of the two ACS/WFC chips per image). Next, the calcsky task is used to create a sky image corresponding to each individual science image. Since we ultimately found that setting FitSky=2, in which the sky is fit inside the PSF radius but outside the photometry aperture, yielded the best photometry, we use the higher-resolution positive step size ( $\mathrm{step}=2$ for WFC3/IR or 4 for ACS/WFC) when generating the sky images.

After preprocessing, initial Dolphot photometry runs were performed on the ACS/WFC and WFC3/IR images separately. This is due in part to offsets of as much as $\sim 1 \operatorname{arcsec}$ in the world coordinate system (WCS) information contained in the headers of the ACS/WFC versus WFC3/IR images, hampering simultaneous alignment of all images to a single reference frame. Therefore, relatively bright $(F 160 W<20)$ stars passing our photometric quality cuts (see below) were cross-matched between the two catalogs from the separate initial Dolphot runs to calculate a positional offset between the ACS/WFC and WFC3/IR distortion corrected reference frames. This positional offset was measured using an iterative 5-sigma clip until convergence was indicated by a change of $<1$ mas in positional offset. The final number of stars used for this calculation ranged from 2008 to 6851 across our target clusters (with a median of 4028), and rms deviation of the final offset among the stars used for matching had a median of 7.1 mas across our cluster sample, with a standard deviation of 1.9 mas. The final positional shifts for each cluster were then applied to the CRVAL1 and CRVAL2 header keywords of all of the WFC3/IR images to place them on the same relative astrometric reference frame as the ACS/WFC images. At this point, a final photometry run was performed on all images simultaneously, using a deep drizzled, cosmic ray cleaned, distortion corrected ACS/WFC .drc image as a positional reference frame (brightness measurements are not performed on the drizzled reference image, rather they are calculated exclusively from the ensemble of individual .flc or .flt science exposures).

In light of the difference in pixel scale and field of view between ACS/WFC and WFC3/IR, the improvement in photometric accuracy and completeness gained by leveraging multiple images together depends critically on the ability to accurately align all individial science images via a common reference frame. Therefore, we searched for the best set of Dolphot alignment parameters by varying the values of UseWCS, Align, AlignTol, AlignStep and AlignIter in search of the smallest alignment rms. The best final set of alignment parameters is given in Table 2 for parameters where the optimal adopted value differed from the recommendation of the Dolphot manual. The total number of stars used for alignment varied between 2667 and 50109 per cluster, and histograms of the alignment rms across our full sample of target clusters is shown in Fig. 3. There, it is apparent that the ACS/WFC images typically yielded the smallest alignment rms relative to the reference frame due to their increased spatial resolution. Furthermore, the long ACS/WFC exposures typically have a smaller alignment rms than the short exposures due to their increased photometric depth (i.e. larger sample size). Meanwhile, the WFC3/IR exposures yield a larger alignment rms in some cases due to their lower spatial resolution, and we found that among the IR exposures with an alignment rms $>10$ mas, $78 \pm 16 \%$ were in the $F 110 \mathrm{~W}$ filter, whereas all $F 160 \mathrm{~W}$ images had an alignment rms $<12.2$ mas, contributing to the bimodality seen in Fig. 3. A likely cause of this behavior could be the increase of the PSF size (i.e. full width at half maximum) with wavelength (Dressel 2018), causing the PSF to be increasingly undersampled in $F 110 W$, but otherwise we found that neither the alignment rms nor the per-cluster difference between $F 110 W$ and $F 160 W$ alignment rms correlated with exposure time or total number of stars used for alignment, demonstrated by $-0.1<\rho<0$ and p-values $>0.5$ from a Spearman rank correlation.

\subsection{PSF Photometry}

Recently, a detailed description of the use of Dolphot to generate deep, multi-band, multi-instrument photometric catalogs was presented by Williams et al. (2014) in the context of the Panchromatic Hubble Andromeda Treasury (PHAT). In that study, extensive artificial star tests were performed to select an optimal set of Dolphot PSF photometry parameters and subsequent cuts on photometric quality parameters (output by Dolphot) to optimize their CMDs given the crowded nature of many of their fields. However, rather than adopt their best values wholesale, we independently validate their choice of photometry parameter values because the nature of our targets and observation strategy differ from theirs in several critical ways. First, although some of the PHAT fields are extremely crowded, they are much less severely plagued by saturated stars and their diffraction spikes. This is due to both the more distant nature of their targets, as well as the moderate Galactic latitude of their target sightlines through the Galaxy. Conversely, all of our target GGCs are more nearby and located close to the Galactic plane, increasing the incidence of both saturated cluster giants and saturated foreground disk and/or bulge stars. Second, our observation strategy differs from that of PHAT since the optical and near-infrared observations of our target clusters attain increasingly 


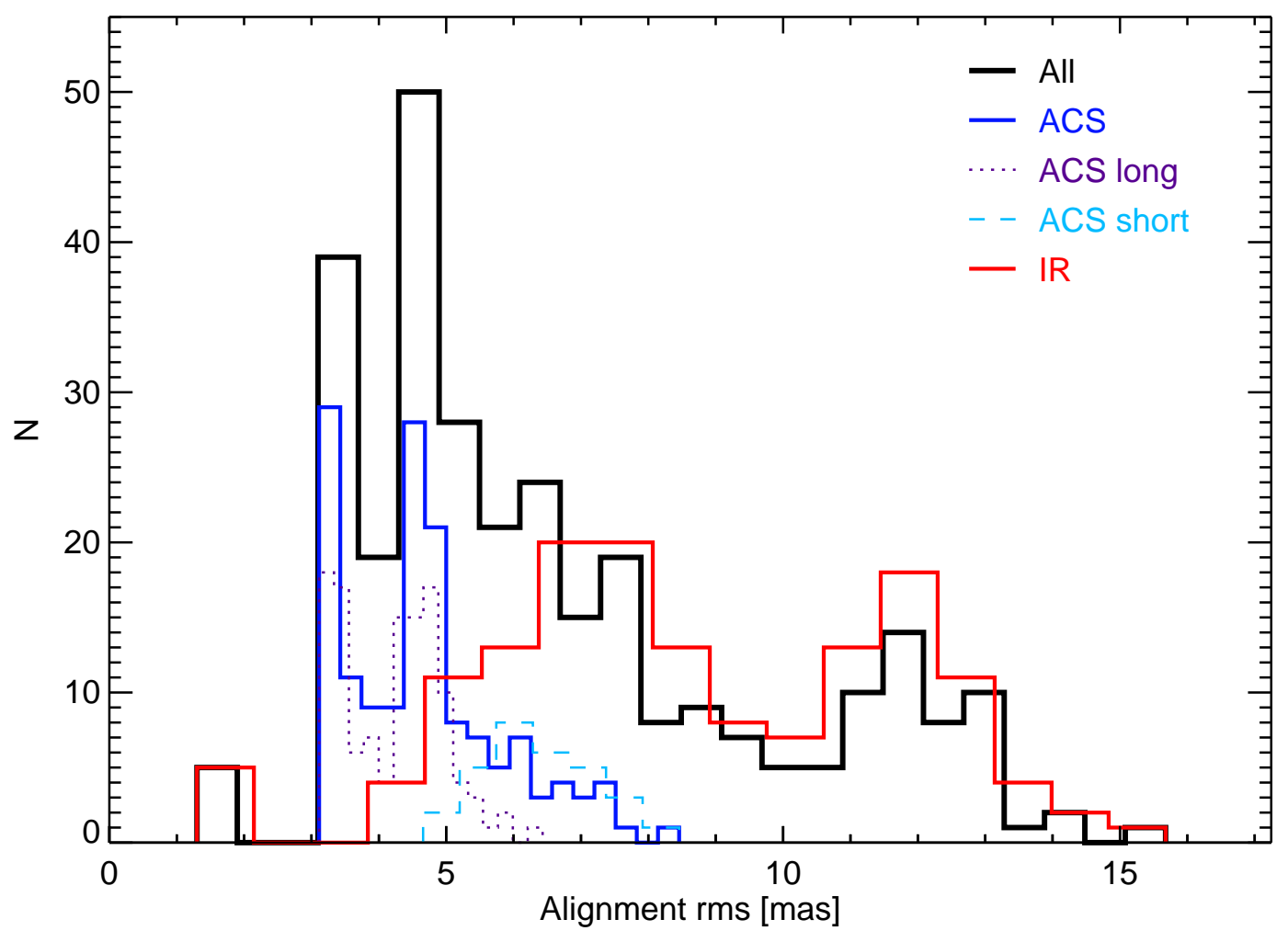

Figure 3. Histograms of the Dolphot initial alignment rms over all images of all target clusters. Different line thicknesses, styles and colors are used to indicate various subsamples (shown using different bin sizes for clarity) as indicated in the upper right-hand corner.

different photometric depths for more extincted clusters. Furthermore, our observations are obtained over a single orbit per instrument so that large dithers and a variety of roll angles are not available, for instance, to rederive distortion solutions.

Given these differences, we chose to validate the parameters used for PHAT by performing a series of Dolphot photometry runs and artificial star tests where parameters were varied, both in turn and in combination, between the values recommended in the Dolphot manual and those used for PHAT. Based on these tests, the final set of Dolphot parameters which we adopted is a mix of the default values and the PHAT values, and all parameters for which we employed values differing from their defaults are listed in Table 2.

\subsection{Photometric Quality Cuts}

Our primary science goals, including the measurement of cluster luminosity and mass functions, necessitate that the raw catalogs output by Dolphot are effectively cleaned of spurious detections. The Dolphot catalogs give several diagnostic parameters for each detection, including sharpness sharp, roundness round, the crowding crowd (the brightness increase in magnitudes which would result from contamination by neighboring stars), and a goodness-of-fit parameter chi, which the Dolphot manual warns may not be trustworthy in an absolute sense (although we employ it in a relative sense below). For each detected source, each of these parameters is given as a global value per source from the combined photometry across all images, and individual per-filter values are also output for each filter in which a source is detected. We employ two different sets of photometric quality criteria depending on instrument, one for the ACS/WFC F606W photometry and one for the WFC3/IR photometry, and apply these cuts in each filter separately using the per-filter diagnostic parameters. This approach proved superior to the use of the global diagnostic parameters reported by Dolphot because of the differences in pixel scale between instruments as well as varying difference in photometric depth between instruments as a function of crowding and line of sight extinction among the target clusters. 
Table 2. Modified Dolphot Parameters

\begin{tabular}{lcc}
\hline \hline Parameter & WFC3/IR & ACS/WFC \\
\hline img_RAper & 3 & 4 \\
img_RChi & 1.5 & 2.0 \\
img_RSky & 820 & 1535 \\
Force1 & 1 & 1 \\
FitSky & 2 & 2 \\
Align & 4 & 4 \\
RCombine & 1.415 & 1.415 \\
PosStep & 0.1 & 0.1 \\
SigFind & 3 & 3 \\
dPosMax & 2.5 & 2.5 \\
\hline
\end{tabular}

For the ACS/WFC imaging, we found that fixed values were sufficient to exclude spurious detections, including diffraction spikes from saturated stars, while exploiting its improved spatial resolution over WFC3/IR to detect real sources in crowded cluster cores. These fixed values varied slightly from cluster to cluster, based on inspection of the spatial and CMD loci of kept and rejected sources, particularly ensuring that the maximum allowed value of crowd is set sufficiently low to exclude diffraction spikes and cosmetic defects while retaining as many true stellar detections as possible.

For WFC3/IR detections, we found that combinations of fixed cuts in the diagnostic parameters or sigma clipping with magnitude did not yield the most efficient rejection of artifacts. Rather, a combination of the crowd and chi parameters yielded the most straightforward constraints on photometric quality, such that a maximum value of crowd $+(\mathrm{chi} / 3)$ ranging from 1.3 to 2.0 (again, based on inspection of spatial and CMD distributions as well as visual inspection of the images) was applied to each cluster. The ability of this parameter to most directly select well-measured sources is illustrated in Fig. 4. There, we compare the ability of crowd+(chi/3) to crowd, chi and $\mid$ sharp $\mid$ to most directly select the stars with the smallest photometric errors. Artificial stars (see Sect. 3.4) were sorted into equally populated bins in each parameter on the horizontal axis, and the median photometric error given by the artificial star tests was plotted for each bin on the vertical axis. Median errors are shown in terms of each of the two WFC3/IR magnitudes as well as $(F 110 W-F 160 W)$ color, and it is clear that of the four diagnostic parameters, crowd $+($ chi $/ 3)$ shows the most clear monotonic trend with photometric error or, in other words, the best discriminating power to select well-measured stars. Our photometric quality cuts for each filter are summarized in Table 3.

Additional verification of photometric quality cuts can be gained from the artificial star tests themselves. While they quantify photometric incompleteness due to application of the cuts, the distribution of the artificial stars with respect to the various diagnostic parameters also indicates where true stellar detections are expected not to lie. Values of the diagnostic parameters seen only in the observed catalogs but not output by the artificial star tests are therefore indicators of where only false detections exist. The ability of our cuts to retain stars falling in the (diagnostic) parameter space occupied primarily by artificial stars while rejecting sources falling outside this space is illustrated for the example case of Terzan $1^{6}$ in Fig. 5 . There, the left column shows density plots of various diagnostic parameters versus $F 160 \mathrm{~W}$ magnitude for real stars which passed our cuts, and the right column shows density plots of the same parameters, but for all artificial stars (not just those passing the cuts). The loci of observed sources which failed our cuts, on the other hand, are indicated by the grey contours in the left column. While it is apparent that some

\footnotetext{
6 Throughout this section, we use Terzan 1 as an example case because star counts over our target cluster parallel fields in the magnitude range where they are all $>90 \%$ complete $(14 \leq F 160 \leq 16)$ indicate that Terzan 1 has the highest field star density, and therefore represents a relatively pathological case for the effects of crowding. At the other extreme, NGC 6638 has the lowest parallel field stellar density in this magnitude range (more than 7 times lower than Terzan 1).
} 

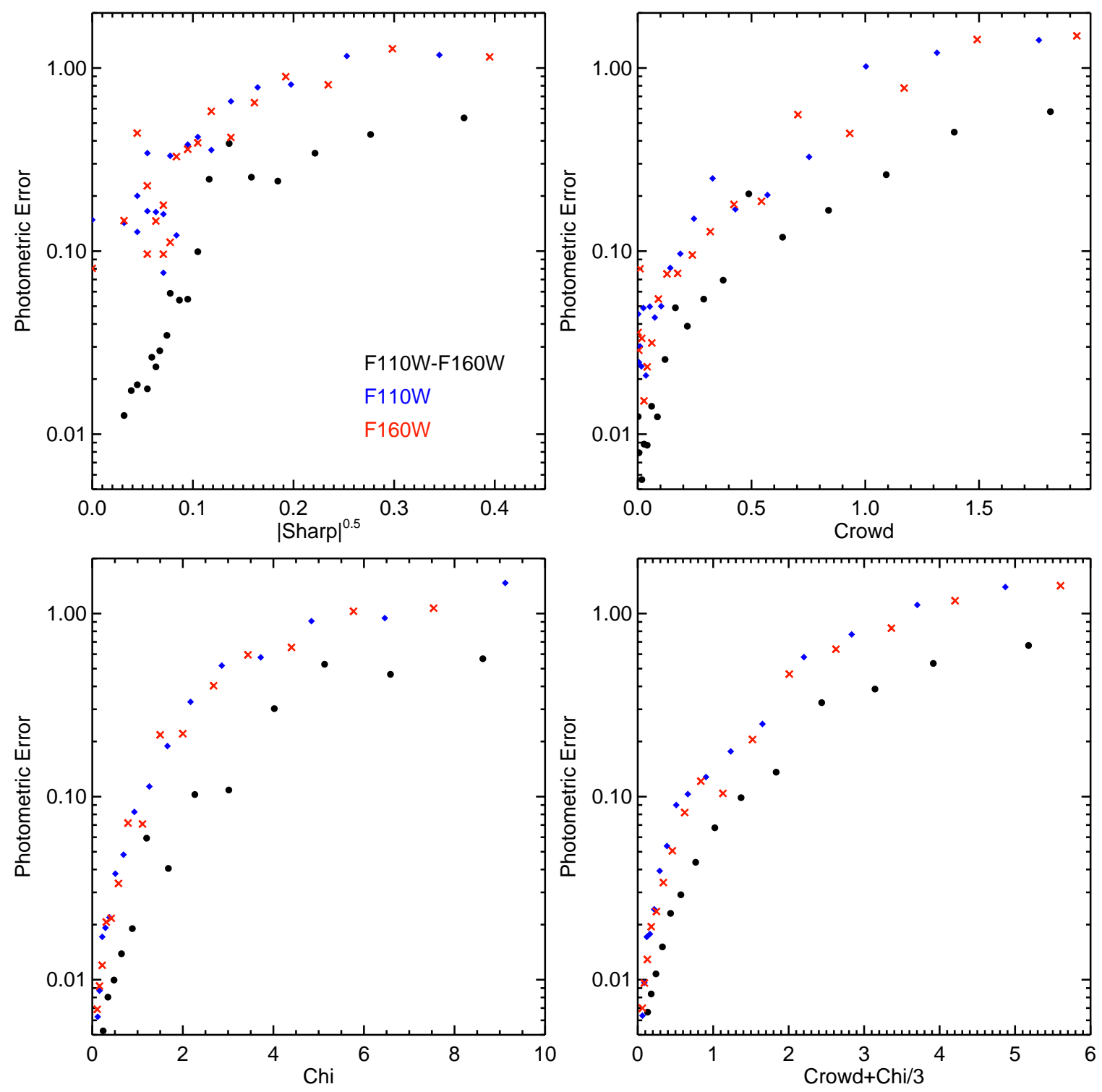

Figure 4. Photometric errors from artificial star tests as a function of several diagnostic parameters, to demonstrate their effectiveness in isolating the most well-measured detections. Median errors in $(F 110 W-F 160 W)$ color is illustrated using black filled points, and $F 110 W$ and $F 160 W$ magnitude is shown using blue diamonds and red crosses respectively. Comparison of the four panels illustrates that crowd $+(\mathrm{chi} / 3)$ maps most clearly to photometric error and is therefore the most useful parameter to selectively eliminate poorly-measured sources with large errors.

stellar sources will be rejected, the similarity in the two distributions demonstrates that the criterion of crowd + chi $/ 3$ does a reasonable, and intentionally somewhat conservative, job of selecting well-measured sources. While the density distributions between the observed and artificial star catalogs appear somewhat dissimilar in high density regions, this is by construction, and is simply a result of the flat input magnitude distribution assigned to the artificial stars. Moreover, while our use of a fixed cut in crowd + chi $/ 3$ effectively imposes a faint magnitude limit, the artificial star tests reveal that at such faint magnitudes, completeness is low, photometric errors are large, and there is a substantial bias in the mean between input and recovered magnitudes (see Sects. 3.4 and 3.5).

The fraction of initially detected sources passing our photometric quality cuts is generally fairly low, particularly in the WFC3/IR filters, where between $8.7 \%$ and $21.8 \%$ of the sources in the WFC3/IR field of view passed our quality cuts in both $F 110 \mathrm{~W}$ and $F 160 \mathrm{~W}$. However, such values are not uncommon in similar applications of Dolphot to produce WFC3/IR photometry in crowded fields via the use of Force1=1 while requiring a thorough rejection 

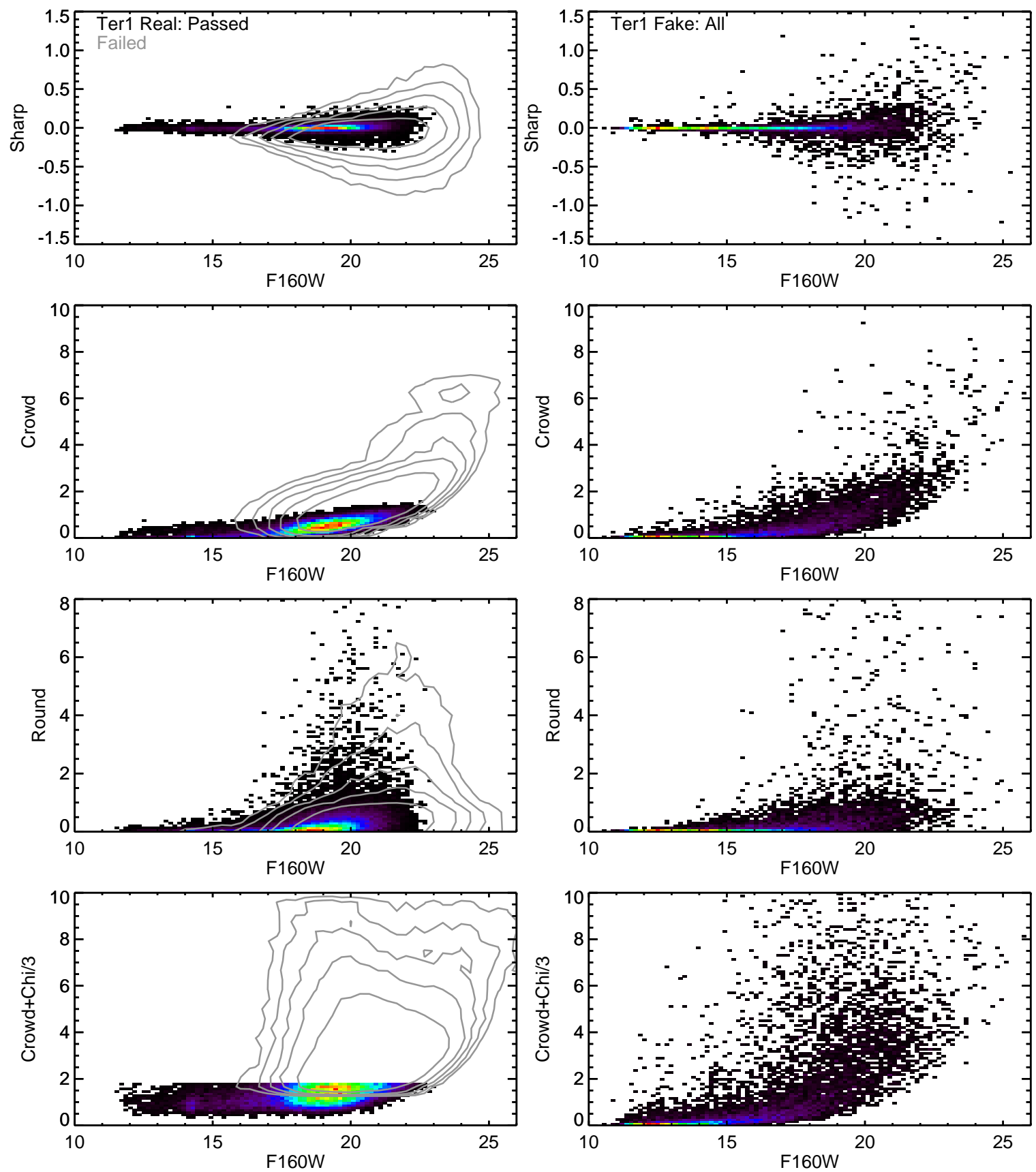

Figure 5. The diagnostic parameters sharp, crowd, round and crowd+chi/3 as a function of F160W magnitude. Left Column: Density plots of stars in our observed catalog for the target cluster Terzan 1 which passed our quality cuts (colored on a linear density scale). The grey contours indicate the loci of stars in the observed catalog which failed our quality cuts, and are plotted at intervals of $(0.0125,0.025,0.05,0.1,0.25)$ times the maximum value to illustrate the full region where rejected sources lie with respect to each parameter. Right Column: Density plots of all artificial stars, shown on the same scale as the left column. Areas which are empty in these plots should be occupied only by spurious detections in the raw observed catalog. The difference in the density distribution between the two columns is partially by construction, since artificial stars were given input $F 160 \mathrm{~W}$ magnitudes with a flat distribution (see Sect. 3.4). 
Table 3. Photometric Quality

Cuts

\begin{tabular}{c}
\hline All Filters \\
\hline Object Type $=1$ \\
Quality Flag $\leq 2$ \\
\hline$F 606 W$ \\
\hline$|\operatorname{sharp}| \leq 0.3$ \\
$0.3 \leq \max (\mathrm{crowd}) \leq 1.0$ \\
$2 \leq \min (\mathrm{S} / \mathrm{N}) \leq 10$ \\
\hline$F 110 W, F 160 W$ \\
\hline $1.3 \leq \max (\operatorname{crowd}+$ chi $/ 3) \leq 2$ \\
$5 \leq \min (\mathrm{S} / \mathrm{N}) \leq 10$ \\
\hline
\end{tabular}

of spurious detections (e.g. Dieball et al. 2016). Furthermore, the possibility that our cuts are overly aggressive is unlikely based on the high $(>90 \%)$ completeness of the WFC3/IR photometry over a range of at least $\sim 4.5$ mag for all target clusters (see Sect. 3.4). Meanwhile, the fraction of sources passing our cuts in both WFC3/IR filters but failing in $F 606 \mathrm{~W}$ showed large variations (ranging from $7 \%$ to $51 \%$ ) which correlate, predictably, with the amount of total and differential extinction seen in the cluster CMDs. Of the sources detected in $F 606 \mathrm{~W}$, less variation is seen, and the fraction passing our cuts ranges from $15.4 \%$ to $38.5 \%$.

\subsection{Artificial Star Tests and Completeness}

Artificial star tests are used to quantify incompleteness as well as photometric and astrometric uncertainties as a function of position, color and magnitude. Artificial stars are assigned input magnitudes drawn from a flat luminosity function in $F 160 W$, and for each of these stars, its color in $(F 606 W-F 160 W)$ and $(F 110 W-F 160 W)$ is randomly drawn from the colors of real stars within $\pm 1 \mathrm{mag}^{7}$ in $F 160 \mathrm{~W}$. The positions of the artificial stars are concentrated towards the cluster center as are the real stars, so that the completeness ascertained from the full ensemble of artificial stars over any magnitude/color range is a reasonable proxy for the true (radially integrated) value, although we do not assume this to be true in a quantitative sense. In practice, the artificial star positions are drawn randomly from a subset of relatively bright $(F 606 W \lesssim 21)$ real stars before applying small random offsets in position, magnitude and color to ensure adequate input spatial and color-magnitude coverage over the entire observed distribution. Artificial stars are inserted one at a time and PSF photometry is performed identically as for real stars, so that each artificial star is affected by any real neighbors present in the science images, but each artificial star is not affected by other artificial stars. The resulting $50 \%$ and $90 \%$ bright and faint completeness limits after application of the photometric quality cuts described in Sect. 3.3 are listed for each filter, for both the primary and parallel fields, in Table 4.

\subsection{Comparison of Reduction Strategies}

We have also used artificial star tests to quantify the advantages and disadvantages of different reduction strategies. The completeness limits (Table 4) and CMDs we present (Figs. 7-22) employ simultaneous photometry of optical and IR images as described in Sect. 3.1-3.2. However, we have also performed IR-only and ACS-only full reductions in order to compare and assess, for example, whether a particular strategy improves or degrades astrometric versus photometric accuracy. Specifically, for the set of IR images, we performed two Dolphot runs independently of the simultaneous optical+IR run already described. The first was performed with the default preprocessing and parameter values recommended in the Dolphot manual. The second was performed with the optimized parameters given in Table 2 , but without the inclusion of the ACS/WFC images. In the left panel of Fig. 6 , we plot several quantities as a

\footnotetext{
7 At the extreme bright and faint ends of the observed magnitude range, this value is increased up to as much as 2 mag in order to sample the full range of plausible input colors and magnitudes spanning our detection limits.
} 
Table 4. Primary and Parallel Field Completeness Limits

\begin{tabular}{lccccccccccccc}
\hline \hline & \multicolumn{3}{c}{ F606W Primary } & \multicolumn{3}{c}{ F110W Primary } & \multicolumn{5}{c}{ F160W Primary } \\
& $C_{\text {min }}^{90}$ & $C_{\text {max }}^{90}$ & $C_{\text {min }}^{50}$ & $C_{\text {max }}^{50}$ & $C_{\text {min }}^{90}$ & $C_{\text {max }}^{90}$ & $C_{\text {min }}^{50}$ & $C_{\text {max }}^{50}$ & $C_{\text {min }}^{90}$ & $C_{\text {max }}^{90}$ & $C_{\text {min }}^{50}$ & $C_{\text {max }}^{50}$ \\
\hline BH261 & 15.607 & 23.454 & 13.975 & 26.704 & 11.902 & 18.550 & 11.384 & 21.654 & 13.117 & 18.962 & 12.699 & 22.293 \\
Djorg2 & 15.446 & 22.713 & 14.802 & 26.295 & 11.700 & 17.118 & 11.260 & 20.048 & 13.151 & 18.408 & 12.640 & 21.082 \\
FSR1735 & 16.251 & 26.517 & 15.487 & 27.673 & 11.816 & 18.912 & 11.350 & 21.427 & 13.183 & 20.170 & 12.745 & 23.027 \\
HP1 & 16.122 & 23.267 & 15.487 & 26.243 & 11.829 & 17.854 & 11.276 & 19.658 & 13.043 & 18.912 & 12.611 & 20.725 \\
NGC6540 & 15.396 & 22.596 & 14.074 & 26.398 & 11.689 & 17.391 & 11.116 & 20.198 & 13.132 & 18.147 & 12.684 & 21.066 \\
NGC6569 & 15.197 & 21.617 & 14.597 & 25.438 & 11.864 & 17.135 & 11.426 & 19.883 & 12.953 & 18.079 & 12.634 & 20.679 \\
NGC6638 & 14.318 & 20.703 & 13.833 & 24.871 & 11.715 & 16.141 & 11.187 & 19.030 & 13.066 & 16.929 & 12.674 & 19.925 \\
Pal6 & 15.972 & 23.658 & 15.288 & 26.516 & 11.721 & 16.194 & 11.103 & 19.178 & 13.283 & 17.711 & 12.692 & 20.559 \\
Ter1 & 16.079 & 23.907 & 15.155 & 26.740 & 11.736 & 15.599 & 11.205 & 18.385 & 13.266 & 17.245 & 12.649 & 19.905 \\
Ter2 & 16.396 & 24.135 & 15.919 & 27.178 & 11.726 & 17.444 & 11.151 & 19.859 & 13.116 & 18.516 & 12.644 & 21.361 \\
Ter4 & 16.501 & 25.614 & 15.801 & 26.853 & 11.780 & 17.668 & 11.189 & 20.057 & 13.399 & 18.889 & 12.716 & 21.595 \\
Ter6 & 16.798 & 24.763 & 15.831 & 26.877 & 11.758 & 16.253 & 11.200 & 19.429 & 13.243 & 17.797 & 12.479 & 20.881 \\
Ter9 & 16.767 & 23.866 & 15.579 & 27.104 & 11.563 & 16.808 & 11.102 & 18.916 & 13.127 & 18.447 & 12.585 & 20.984 \\
Ter10 & 16.601 & 24.996 & 16.000 & 27.377 & 11.739 & 17.767 & 11.212 & 20.276 & 13.345 & 18.865 & 12.658 & 21.200 \\
Ter12 & 16.519 & 25.086 & 15.763 & 27.452 & 11.677 & 17.181 & 11.202 & 19.829 & 13.255 & 18.668 & 12.775 & 21.480 \\
Ton2 & 16.219 & 23.665 & 15.504 & 27.152 & 11.783 & 17.834 & 11.314 & 20.500 & 13.227 & 18.884 & 12.696 & 21.971
\end{tabular}

\begin{tabular}{|c|c|c|c|c|c|c|c|}
\hline \multicolumn{4}{|c|}{ F110W Parallel } & \multicolumn{4}{|c|}{ F160W Parallel } \\
\hline$C_{\min }^{90}$ & $C_{\max }^{90}$ & $C_{\min }^{50}$ & $C_{\max }^{50}$ & $C_{\min }^{90}$ & $C_{\max }^{90}$ & $C_{m i n}^{50}$ & $C_{\max }^{50}$ \\
\hline 11.654 & 15.773 & 11.243 & 21.773 & 13.542 & 18.916 & 12.863 & 22.632 \\
\hline 11.934 & 16.831 & 11.219 & 19.700 & 13.705 & 16.928 & 12.971 & 20.549 \\
\hline 11.934 & 17.981 & 11.313 & 23.133 & 13.770 & 19.100 & 13.014 & 24.397 \\
\hline 12.144 & 17.161 & 11.525 & 20.027 & 13.440 & 18.245 & 12.907 & 21.186 \\
\hline 12.028 & 16.992 & 11.192 & 20.280 & 13.528 & 17.460 & 12.872 & 21.218 \\
\hline 12.052 & 17.733 & 11.424 & 23.647 & 13.670 & 18.361 & 12.953 & 24.532 \\
\hline 11.773 & 18.427 & 11.197 & 23.340 & 13.851 & 19.244 & 13.169 & 24.731 \\
\hline 12.071 & 14.734 & 11.403 & 19.827 & 13.537 & 17.066 & 12.861 & 20.896 \\
\hline 11.889 & 16.776 & 11.312 & 19.717 & 13.605 & 16.969 & 12.719 & 21.317 \\
\hline 11.907 & 16.010 & 11.268 & 20.315 & 13.570 & 18.275 & 12.824 & 21.515 \\
\hline 11.933 & 15.829 & 11.263 & 20.604 & 13.462 & 18.003 & 12.627 & 22.052 \\
\hline 11.864 & 16.157 & 11.300 & 19.964 & 13.542 & 18.025 & 12.905 & 21.567 \\
\hline 11.922 & 16.989 & 11.350 & 20.283 & 13.492 & 17.587 & 12.911 & 21.545 \\
\hline 11.889 & 16.518 & 11.357 & 20.603 & 13.591 & 16.768 & 12.826 & 21.880 \\
\hline 12.107 & 16.631 & 11.443 & 21.090 & 13.632 & 19.012 & 12.971 & 22.597 \\
\hline 12.149 & 18.678 & 11.401 & 22.860 & 13.649 & 18.527 & 13.000 & 24.683 \\
\hline
\end{tabular}

Note-For each filter, $C_{\min }^{90}$ and $C_{\max }^{90}$ denote the $90 \%$ bright and faint completeness limits, while $C_{\min }^{50}$ and $C_{\max }^{50}$ denote the $50 \%$ bright and faint completeness limits. All values are magnitudes in the Vegamag photometric system native to each instrument as described in Sect. 4 . 
function of input $F 160 \mathrm{~W}$ magnitude for each of the three (including the original ACS+IR run) Dolphot runs. In the top panel, we plot the completeness fraction, and also give the number of stars passing the photometric quality cuts described in Sect. 3.3. In the second and third panels, we plot the median (thin line) and $\pm 1 \sigma$ (thick lines) difference in $F 160 W$ magnitude and $(F 110 W-F 160 W)$ color. In the bottom panel, we plot the positional error as a function of magnitude. In a practical sense, the positional error given here is more appropriately viewed as a lower limit since Dolphot assumes the same distortion solution to place artificial stars on individual images as well as transform detections in these images to the reference image frame.

As a guide, we have used the values in the top panel to indicate the $50 \%$ and $90 \%$ completeness limits for each of the three runs as dotted and dashed vertical lines. In the right-hand panel of Fig. 6, we show the results of an analogous experiment for the ACS/WFC imaging, where we compare the ACS+IR reduction strategy to ACS-only Dolphot runs using both the default parameters and our optimized parameters. Taking both panels of Fig. 6 together, several results are apparent:

1. For any given run, the magnitude offset (bias) never exceeds zero at a significant $(>1 \sigma)$ level, over all magnitudes within the $50 \%$ completeness limits. The only exception to this rule is the brightest stars in the ACS/WFC images, which are only recovered when photometered simultaneouly with WFC3/IR. However, in this case, they show a median offset of $\sim 0.05 \mathrm{mag}$ in $F 606 \mathrm{~W}$ and no offset in WFC3/IR magnitude or color.

2. Unsurprisingly, the positional accuracy of WFC3/IR detections is improved when photometered simultaneously with ACS/WFC. Similarly, the positional accuracy of ACS/WFC is degraded with the inclusion of WFC3/IR. However, even in this case, the internal positional errors for bright, unsaturated sources are about 0.5 mas, or 0.01 ACS/WFC pix, roughly consistent with what has been feasible by Bellini et al. (2014) per epoch with a similar ACS/WFC observing strategy.

3. In the default run for WFC3/IR, and to a lesser extent, the IR-only run, a smaller number of sources are detected and completeness is lower at a given $F 160 \mathrm{~W}$ magnitude. At face value, this could simply be suggesting that our quality cuts are overly harsh in these cases. However, for these runs, the photometric errors in color and magnitude are not smaller, and are in fact larger, than those from the ACS+IR photometry at a given magnitude, indicating that the ACS+IR photometry does in fact recover a greater number of sources with smaller photometric errors.

4. The ACS-only runs appear to provide photometry which is of equal quality and greater astrometric precision than the ACS+IR runs, with the exception of sources near the saturation limit mentioned above. However, significant magnitude offsets are seen in all cases faintward of the $50 \%$ completeness limit which we were unable to further mitigate using photometric quality cuts. Therefore, the $F 606 \mathrm{~W}$ photometry should not be considered trustworthy in a quantitative sense at such faint magnitudes, even in light of photometric errors of $>0.1$ mag.

The results we present in Sect. 4 are based on a simultaneous Dolphot reduction of the ACS and IR images together, since the artificial star tests using this strategy (shown in red in Fig. 6) reveal the best combination of dynamic range and photometric precision compared to the other strategies for the purpose of constructing clean, multi-band photometric catalogs. However, it is also clear that specific science goals may benefit (for example, in positional accuracy) from tailored alternate data reduction strategies.

\subsection{Astrometric Calibration}

The final cleaned, distortion corrected photometric catalogs are astrometrically calibrated using our 2MASScalibrated VVV PSF photometry catalogs. Given the large and variable optical extinction towards many of our target clusters, near-IR photometry is needed to provide a sufficient number of calibrating stars in the field of view of our observations. Furthermore, our VVV PSF catalogs provide significant advantages over 2MASS in terms of both photometric depth and spatial resolution (Mauro et al. 2012; Cohen at al. 2014, 2017), rendering them vastly more compatible with the HST imaging obtained here. Briefly, the astrometric calibration for each target cluster was performed as follows: First, only bright $\left(K_{S} \lesssim 15\right)$, unsaturated sources detected in multiple bands in the VVV catalogs are considered for use as astrometric standards. Given offsets of over 1 arcsec seen in the header WCS of the $H S T$ reference image, we use a simple matching algorithm with a $5 \sigma$ clip (as described in Sect. 3.1 but with a more generous convergence criterion of $\left.0.01^{\prime \prime}\right)$ to cross reference distortion-free pixel coordinates of HST detections with 

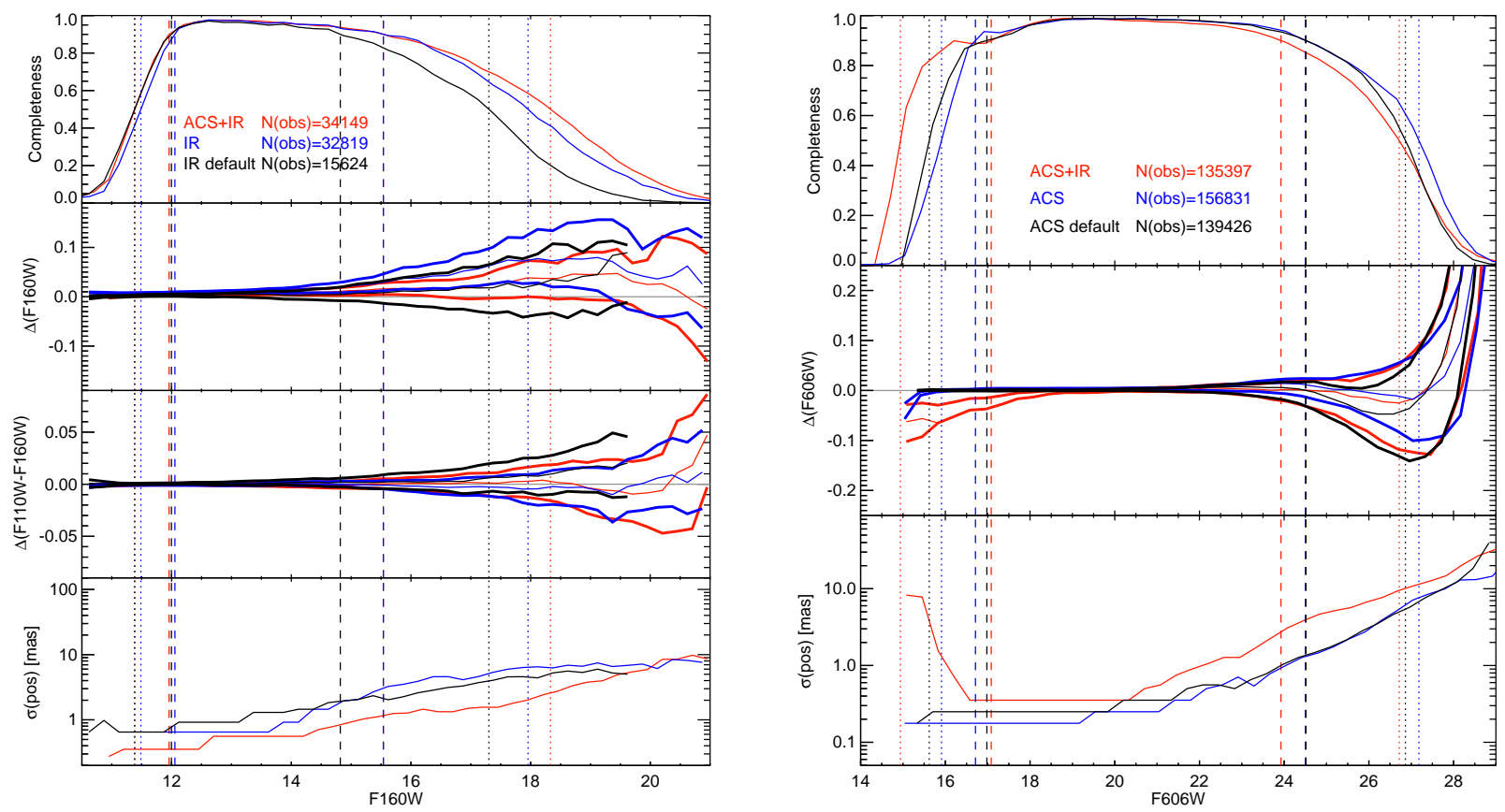

Figure 6. Comparison of photometric and astrometric accuracy from different Dolphot data reduction strategies using artificial star tests, for WFC3/IR images (left column) and ACS/WFC images (right column) of Terzan 1. In each plot, we compare three reduction strategies: The default recommended Dolphot preprocessing and photometry parameters (black), our optimized parameters for each instrument separately (blue), and optimized parameters used to perform simultaneous photometry of ACS and IR images together (red). For each of the three strategies, 50\% and $90 \%$ completeness limits are shown as dotted and dashed vertical lines respectively.

(2MASS-calibrated) RA and Dec of VVV detections. Lastly, the IRAF ccmap and cctran tasks are used (rejecting outliers with a $3 \sigma$ clip) to calculate and apply the final astrometric solution. The number of stars used as astrometric calibrators varied between 605 and 1995 among our target clusters, and the astrometric rms deviation among these calibrators was $\leq 0.025^{\prime \prime}$ per coordinate in all cases.

\section{COLOR-MAGNITUDE DIAGRAMS}

Here, we present the observed CMDs of all 16 target clusters, in several different color-magnitude planes. The magnitudes and colors reported here have had aperture corrections applied by Dolphot and are placed on the Vegamag system using ACS/WFC zeropoints and encircled energy corrections from Bohlin (2012) and WFC3/IR values posted on the STScI website ${ }^{8}$. For each CMD, we use dashed and solid grey lines to illustrate the radially integrated $50 \%$ and $90 \%$ completeness limits. For each of the four color-magnitude planes, the scales of the color and magnitude axes are kept fixed from cluster to cluster to facilitate a comparison (although the values of the axes may shift). We now move on to a discussion of the individual clusters, summarizing previous literature studies in each case. This is done primarily to emphasize the lack of previous studies of these clusters, but also to serve as a basis for comparison of cluster distances, reddenings and metallicities. Remarkable effort has been made to place these values on a self-consistent footing to the extent possible by H96, which is the only catalog to do so. However, their reliance (by necessity) on uncorrected optical photometry (to obtain distances, structural parameters, and in some cases metallicities), along with the assumption of a constant, standard optical extinction law, may cause systematic biases in the resulting values. For this reason, we revisit the earlier studies of each target cluster as completely as practically feasible, so that their methodology can be examined on a case-by-case basis.

$$
\text { 4.1. } B H 261=A L 3=E S O 456-78
$$

8 www.stsci.edu/hst/wfc3/analysis/ir_phot_zpt 
Essentially all that is known about this cluster photometrically is based on the discovery paper of Ortolani et al. (2006), utilizing optical BVI photometry, although a King (1962) profile fit to statistically cleaned 2MASS photometry was performed by Bonatto \& Bica (2008b). Based on radial cuts to their photometric catalog, Ortolani et al. (2006) claim that the cluster is quite sparse with a blue $\mathrm{HB}$, and obtain cluster parameters via a direct comparison of the RGB and HB sequences to those of the GGC M 5 . They assume a standard $\left(R_{V}=3.1\right)$ extinction law to find $R_{\odot}=6.5 \pm 0.5$ kpc, $E(B-V)=0.36 \pm 0.03$ and a photometric metallicity estimate of $[\mathrm{Fe} / \mathrm{H}]=-1.3 \pm 0.25$. Proper motion cleaning by Rossi et al. (2015) supports the blue HB morphology and they find a large proper motion in Right Ascension $(4.77 \pm 0.46 \mathrm{mas} / \mathrm{yr})$ compared to the background bulge/disk population, but as this cluster has no published radial velocities, they are unable to constrain its three-dimensional motion. We confirm the sparse blue HB extending faintward to $F 160 W \sim 16$ at $(F 110 W-F 160 W) \sim 0.1$ and $(F 606 W-F 160 W) \sim 0.5$ in Fig. 7 . As in the case of the majority of our target clusters, our imaging appears to extend well faintward of the lower main sequence knee (LMSK), seen near $F 160 W \sim 20$ in this case. However, careful measurement of field contamination is needed to reveal to what extent this feature is comprised of bulge field stars superimposed on the cluster main sequence, although in this case we may be seeing largely cluster members given that this cluster lies $>5$ degrees from the Galactic plane.

\section{2. $\operatorname{Djorg} 2=E S O 456-S C 38$}

Several studies of this cluster give a range of parameters. Based on a comparison of optical photometry to the GGC 47 Tuc, Ortolani et al. (1997a) give $R_{\odot}=5.5 \pm 0.8 \mathrm{kpc}, E(B-V)=0.89 \pm 0.08$ and a photometric metallicity estimate of $[\mathrm{Fe} / \mathrm{H}] \sim=-0.5$. Meanwhile, Valenti et al. (2010) use near-IR photometry to obtain $R_{\odot}=7.0 \mathrm{kpc}, E(B-$ $V)=0.94$ and a photometric metallicity of $[\mathrm{Fe} / \mathrm{H}]=-0.65$. Based on low-resolution optical spectra of four radial velocityselected members, Dias et al. (2016) report $[\mathrm{Fe} / \mathrm{H}]=-0.79 \pm 0.09$, although a discrepancy of $\sim 0.7$ dex between the values they obtain from empirical versus synthetic spectral libraries, in addition to the small number of members available, suggests that an independent spectroscopic metallicity estimate would be valuable in this case, especially given the evidence of a blue HB extending to $F 160 W \sim 16.5$ in Fig. 8, unusual at such a high metallicity. A proper motion of $(3.08,2.00) \pm(0.29,0.34) \mathrm{mas} / \mathrm{yr}$ in RA and Dec respectively is given by Rossi et al. (2015).

\section{3. $F S R 1735=2 M A S S$ GC-03}

The idea that this cluster candidate was in fact a GGC was put forth by Froebrich et al. (2007) based on near-IR photometry. They give $R_{\odot}=9.1 \pm 1.0 \mathrm{kpc}$ based on the $K$-band magnitude of the red clump, using $A_{K}=0.5 \pm 0.1$, and a photometric metallicity of $[M / H]=-0.8 \pm 0.1$ based on the slope of the upper RGB. Recently, Carballo-Bello et al. (2016) used subsequent near-IR photometry and spectroscopy to obtain $R_{\odot}=10.8 \pm 0.9 \mathrm{kpc}$ based on time series imaging of three ab-type RR Lyrae variables, and a spectroscopic metallicity of $[\mathrm{Fe} / \mathrm{H}]=-0.9 \pm 0.2$ from five likely members. Despite substantial differential reddening, evidence of a blue HB extending to $F 160 W>18$ is visible in Fig. 9, and comparison of the primary and parallel fields immediately reveals that in this case the primary source of contamination is the foreground Galactic disk rather than the bulge given the Galactic longitude of this cluster.

$$
\text { 4.4. HP } 1=\text { ESO 455-11 = Gcl 67 }
$$

Infrared photometry of this cluster and spectroscopy of six red giants was obtained by Minniti et al. (1995), finding $E(J-K)=0.94 \pm 0.10$ and $[\mathrm{Fe} / \mathrm{H}]=-0.3 \pm 0.2$, somewhat higher than $[\mathrm{Fe} / \mathrm{H}]=-0.56$ from the integrated light spectroscopic database of Armandroff \& Zinn (1988). Using VI photometry, Ortolani et al. (1997b) give $R_{\odot}=6.85 \pm 0.2 \mathrm{kpc}$ and estimate $[\mathrm{Fe} / \mathrm{H}] \sim-1.5$ based on a direct comparison to NGC 6752 , pointing out the extensive blue HB and proximity to the Galactic center, suggesting that early high values of $[\mathrm{Fe} / \mathrm{H}]$ were due to contamination by field stars. Using near-IR photometry, Davidge (2000) performed a comparison to M 13, giving $E(B-V)=0.74$ and $(m-M)_{0}=14.3$, corresponding to $R_{\odot} \sim 7.25 \mathrm{kpc}$, while medium resolution $K$-band spectroscopy by Stephens \& Frogel (2004) led to $[\mathrm{Fe} / \mathrm{H}]=-1.30 \pm 0.09$. The compilation of near-IR bulge GGC photometry by Valenti et al. (2010) gives $R_{\odot}=6.8 \mathrm{kpc}$ and $[\mathrm{Fe} / \mathrm{H}]_{\mathrm{CG} 97}=-1.12$. A proper motion-cleaned CMD was presented by Ortolani et al. (2011), which they used to estimate distances ranging from $6.8 \leq R_{\odot} \leq 7.5 \mathrm{kpc}$ depending on methodology, and they claim that HP 1 may be one of the oldest GGCs based on its location in HB morphology-metallicity space. High resolution optical spectra have been obtained by Barbuy et al. $(2006,2016)$, who give $[\mathrm{Fe} / \mathrm{H}]=-1.06 \pm 0.10$ based on eight radial velocity members, in good agreement with $[\mathrm{Fe} / \mathrm{H}]=-1.17 \pm 0.07$ from low-resolution spectra of eight members by Dias et al. (2016), who also claim that HP 1 is a likely candidate to be the oldest bulge GGC. We confirm the extended blue HB described by Ortolani et al. (2011), the most clearly visible among our sample, which is apparent in all three of the color-magnitude planes shown in Fig. 10 and has a tail extending from $F 606 W \sim 18.5$ to at least $F 606 W \sim 20.5$. 


\section{5. $N G C 6540=$ Djorg 3}

Average values of $E(B-V)=0.59 \pm 0.11$ and $R_{\odot}=3.5 \pm 0.4 \mathrm{kpc}$ were estimated by Bica et al. (1994), who noted the blue horizontal branch and, based on optical photometry and integrated spectroscopy, claimed that this cluster is very concentrated and possibly post core collapse. Using optical echelle spectra of six stars, Côté (1999) estimated $[\mathrm{Fe} / \mathrm{H}]=-1.4$ and a mean radial velocity of $-17.7 \pm 1.4 \mathrm{~km} / \mathrm{s}$. This cluster was observed in the Piotto et al. (2002) WFPC2 snapshot program, and the next photometric study was the near-infrared survey of bulge GCs by Valenti et al. (2010), who also noted the sparseness of the cluster RGB and the concentrated nature of the cluster. Their photometric calibrations and observed values of the RGB slope and RGB tip led to values of $[\mathrm{Fe} / H]_{C G 97}=-1.29, E(B-V)=0.66$ and $R_{\odot}=5.2 \mathrm{kpc}$. Proper motions of NGC 6540 are included in Rossi et al. (2015), who suggest that the cluster may be near apogalacticon based on the combination of low observed radial velocity and small proper motions which they measure relative to the foreground population. However, they also point out the difficulty of proper motion cleaning in this particular case, and list a shorter adopted distance of $R_{\odot}=3.7 \pm 0.3 \mathrm{kpc}$. We confirm the blue HB extending to $F 160 W \sim 16$ in Fig. 11, although the $(F 606 W, F 606 W-F 160 W)$ CMD illustrates that it lacks the extended tail seen in HP1. Comparison of the primary and parallel field CMDs also suggests that, as with BH261, this cluster is relatively sparsely populated compared to the remainder of the target GGCs.

\section{6. $N G C 6569$}

Optical $B V I$ photometry was presented by Ortolani et al. (2001b), who estimated $E(B-V)=0.53$ and $R_{\odot}=9.8 \pm 1.0$ kpc based on a comparison to 47 Tuc. The compilation of Valenti et al. (2010) lists $E(B-V)=0.49$ and a longer distance of $R_{\odot}=12.0 \mathrm{kpc}$, and Valenti et al. (2011) found [Fe/H]=-0.79 \pm 0.02 based on high resolution near-IR spectroscopy of six cluster giants. While optical photometry was available from the Piotto et al. (2002) catalog, a double horizontal branch red clump was reported for the first time by Mauro et al. (2012) based on subsequent deeper near-IR photometry from the VVV survey. The metallicity of NGC 6569 remains somewhat controversial, and this cluster is a $3 \sigma$ outlier in the Mauro et al. (2014) calibration of CaII triplet reduced equivalent width versus metallicity. The CMD morphology in the near-IR similarly suggests a lower metallicity than the spectroscopic value $([\mathrm{Fe} / \mathrm{H}]=-1.00 \pm 0.05$; Cohen at al. 2017). At the same time, Dias et al. (2016) give $[\mathrm{Fe} / \mathrm{H}]=-0.66 \pm 0.07$ based on low resolution optical spectroscopy of seven members. Recent high-resolution spectroscopy of a larger sample of cluster members by Johnson et al. (2018) found $[\mathrm{Fe} / \mathrm{H}]=-0.87$ and evidence against metallicity or light-element variations as the cause of the double HB. Meanwhile, cluster RR Lyrae variables have been recognized at least since Hesser et al. (1984), and a detailed variability analysis by Kunder et al. (2015) found that there was no evidence for helium enhancement among the cluster RR Lyrae. As in the case of FSR1735, comparison of the primary and parallel fields in Fig. 12 suggests that field contamination towards this cluster is fairly minimal given its distance from the Galactic plane. In addition, the HB is seen to extend significantly blueward of the HB red clump, although the optical-IR CMDs do not reveal a blue HB extention as dramatic as seen in HP 1 or even NGC 6638.

\section{7. $N G C 6638$}

A cluster CMD was presented by Alcaino \& Liller (1983), although they cautioned that their distance and reddening estimates should be viewed as tentative. Low-resolution spectroscopy by Smith \& Stryker (1986) of several RR Lyrae discovered by Rutily \& Terzan (1977) yielded a metallicity estimate of $[\mathrm{Fe} / \mathrm{H}]=-0.82 \pm 0.2$. While the CaII tripletbased value of $[\mathrm{Fe} / \mathrm{H}]=-0.95 \pm 0.12$ (Mauro et al. 2014) is in reasonable agreement with the earlier estimate of $[\mathrm{Fe} / \mathrm{H}]=-$ $0.99 \pm 0.07$ (Carretta et al. 2009) and the subsequent photometric estimate of $[\mathrm{Fe} / \mathrm{H}]=-1.09 \pm 0.07$ (Cohen at al. 2017), there have been no high resolution spectroscopic studies of cluster members. As in the previous two cases, this cluster was included in the Piotto et al. (2002) survey due to its relatively low foreground extinction. Near-IR photometry by Valenti et al. (2005) led to estimates of $[\mathrm{Fe} / H]_{C G 97}=-0.97, E(B-V)=0.43$ and $R_{\odot}=10.3 \mathrm{kpc}$. Along with HP1, this cluster presents the most clear evidence of a blue HB (see Fig. 13). However, in this case the blue HB does not have an extensive tail although a small degree of "turnover" beyond the horizontal portion of the HB is seen in the $(F 606 W, F 606 W-F 160 W)$ CMD at $(F 606 W-F 160 W) \sim 1.1, F 606 W \sim 16.5$. This cluster, along with NGC 6569, is among the least affected by differential reddening in our sample.

\subsection{Palomar $6=$ ESO 520-21}

Based on the locus of the HB and RGB in the VI CMD, Ortolani et al. (1995) estimate $E(B-V)=1.33$ and $R_{\odot}=8.95$ kpc assuming $[\mathrm{Fe} / \mathrm{H}] \sim-0.4$. Using near-IR photometry and spectroscopy, Minniti (1995) list $E(J-K)=0.91$ and a 
super-solar metallicity of $[\mathrm{Fe} / \mathrm{H}]=0.2 \pm 0.3$ based on measurements of three giants which they consider to be likely members. Subsequently, near-IR photometry was presented by Lee \& Carney (2002), who estimated $E(B-V)=1.30$ and a distance modulus corresponding to $R_{\odot}=7.2 \mathrm{kpc}$. They also found that their measured value of the RGB slope implies a metallicity of $[\mathrm{Fe} / \mathrm{H}]=-1.2$, significantly lower than previous studies. Near-IR echelle spectroscopy of seven stars (Lee et al. 2004) further constrained their metallicity estimate to $[\mathrm{Fe} / \mathrm{H}]=-1.0 \pm 0.1$, although near-IR spectroscopy of five members by Stephens \& Frogel (2004) led to a much higher value of $[\mathrm{Fe} / \mathrm{H}]=-0.52 \pm 0.2$. Most recently, a value of $[\mathrm{Fe} / \mathrm{H}]=-0.85 \pm 0.11$ was reported from low-resolution optical spectra of four stars considered to be radial velocity members (Dias et al. 2016), although the $[\mathrm{Fe} / \mathrm{H}]$ values which they derive based on empirical versus synthetic spectral libraries differ by more than 1 dex. The red horizontal branch was confirmed by Rossi et al. (2015), who present cleaned CMDs and proper motion measurements. The red HB is also clearly visible in Fig. 14, although differential reddening is particularly discernible from the optical-IR CMDs.

\subsection{Terzan $1=$ ESO 455-23}

Based on optical photometry, Ortolani et al. (1993) estimated a distance of $R_{\odot}=4-5 \mathrm{kpc}$, and noted the differences between the field population close to Terzan 1 and that of Baade's Window. In a subsequent study using HST WFPC2 optical imaging, Ortolani et al. (1999a) estimate $E(B-V)=5.2 \pm 0.5$ and $R_{\odot}=5.2 \pm 0.5 \mathrm{kpc}$, cautioning that this distance depends strongly on the adopted reddening law. They also note that the metallicity inferred from the RGB morphology $([\mathrm{Fe} / \mathrm{H}] \sim-1.2)$ is at odds with the relatively red HB morphology. Optical spectroscopy of 11 velocity members by Idiart et al. (2002) yielded $[\mathrm{Fe} / \mathrm{H}]=-1.27 \pm 0.05$. Most recently, high resolution near-IR spectroscopy of 15 members by Valenti et al. (2015) yielded values of $[\mathrm{Fe} / \mathrm{H}]=-1.26 \pm 0.03$ and high $(\sim 0.4) \alpha$-enhancement, while Valenti et al. (2010) report $E(B-V)=1.99$ and $R_{\odot}=6.6 \mathrm{kpc}$ based on a comparison of near-IR photometry to 47 Tuc. Using two epochs of ground-based photometry, Rossi et al. (2015) claim a very small relative proper motion, suggesting that in combination with the radial velocity reported by Idiart et al. (2002), the cluster could be on an elongated orbit, although Valenti et al. (2015) find a significantly smaller (by a factor of nearly 2) radial velocity. Lastly, it bears mention that x-ray emission has been detected from this cluster (Makishima et al. 1981; Johnston et al. 1995; Borrel et al. 1996; Guainazzi et al. 1999; Molkov et al. 2001; Wijnands et al. 2002), including several sources which are likely located within the cluster along the line of sight (Cackett et al. 2006). From the IR CMDs of the primary and parallel fields in the top row of Fig. 15, it appears that there is a shift in the foreground/background RGB, although a density profile will clarify to what extent the cluster contributes to the reddest RGB stars seen in the target field. Also, the HB may extend significantly blueward of the clump seen at $(F 110 W-F 160 W)=1.2$, possibly as far as $(F 110 W-F 160 W) \sim 0.7$ at $F 160 W \sim 16$, but careful field decontamination is needed to clarify the HB morphology of this cluster.

\subsection{Terzan 2 = ESO 454-29}

Near-IR photometry was used by Christian \& Friel (1992) to estimate $R_{\odot} \sim 10 \mathrm{kpc}$ and $E(B-V)=1.25 \pm 0.15$ via comparison to then-available template GGC giant branches, assuming $[\mathrm{Fe} / \mathrm{H}]=-0.25 \pm 0.15$ from Armandroff \& Zinn (1988). The first optical CMD was presented by Ortolani et al. (1997c), who estimate $E(B-V)=1.57$ and $R_{\odot}=7.7$ kpc assuming a standard reddening law (or shorter distances for non-standard values of $R_{V}>3.1$ ). Based on spectra of seven stars, Stephens \& Frogel (2004) estimate $[\mathrm{Fe} / \mathrm{H}]=-0.87 \pm 0.06$, and Valenti et al. (2010) use their near-IR photometry to estimate $E(B-V)=1.87, R_{\odot}=7.4 \mathrm{kpc}$ and $[\mathrm{Fe} / \mathrm{H}]_{C G 97}=-0.72$. A low proper motion of $\lesssim 1 \mathrm{mas} / \mathrm{yr}$ is given by Rossi et al. (2015) based on two epochs of ground-based optical imaging, and there have been several studies of x-ray sources located towards this cluster (e.g. Barret et al. 1991; Mereghetti et al. 1995; Guainazzi et al. 1998; Barret et al. 1999; Altamirano et al. 2008; Valencic et al. 2009). A short red HB is clearly visible in our CMDs in Fig. 16, although the optical-IR CMDs reveal significant differential reddening.

\subsection{Terzan $4=$ Gcl-66.1}

Ortolani et al. (1997d) estimate $E(B-V)=2.35$ and $R_{\odot}=8.3 \mathrm{kpc}$, noting the blue horizontal branch of this cluster, and subsequent HST NICMOS observations (Ortolani et al. 2001a) are used to calculate a distance of $R_{\odot}=8.0 \pm 0.3$ kpc via direct comparison with the GGC M 92 (Ortolani et al. 2007). Using their database of near-IR photometry, Valenti et al. (2010) report $E(B-V)=2.05$ and $R_{\odot}=6.7 \mathrm{kpc}$. Medium resolution near-IR spectroscopy gives $[\mathrm{Fe} / \mathrm{H}]=-$ $1.62 \pm 0.08$ (Stephens \& Frogel 2004), in excellent agreement with the value of $[\mathrm{Fe} / \mathrm{H}] \sim-1.6$ from high resolution IR spectra of four members by Origlia \& Rich (2004), who also report a high value of $[\alpha / F e] \sim 0.5$. Proper motions from 
Rossi et al. (2015) are relatively large compared to the majority of their sample of GGCs towards the bulge, and they suggest that this, in combination with its relatively low metallicity, implies a possible halo origin for this cluster. A very low concentration parameter of $c=0.9 \pm 0.2$ was inferred from fits of King profiles to 2MASS density and surface brightness profiles (Bonatto \& Bica 2008b). Our CMDs in Fig. 17 illustrate severe differential reddening across the primary field, and as in the case of Terzan 1, a clear change in the location of the bulge RGB between the primary and parallel fields.

\subsection{Terzan $6=$ ESO $455-49$}

Near-IR photometry was presented by Fahlman et al. (1995), who estimated $E(B-V)=2.04$ and $R_{\odot}=6.8 \pm 0.46 \mathrm{kpc}$ based on a direct comparison to the GGC M 71. Shortly afterward, Barbuy et al. (1997) use an optical CMD to estimate similar values of $E(B-V)=2.24$ and $R_{\odot}=7.0 \mathrm{kpc}$ assuming a standard reddening law. The only subsequent photometric study is the near-IR database of Valenti et al. (2010), estimating $E(B-V)=2.35$ and $R_{\odot}=6.7 \mathrm{kpc}$. There is one known low-mass X-ray binary in Terzan 6 (e.g. in’t Zand et al. 2003; Bahramian et al. 2016). As in the case of Terzan 2, our CMDs confirm a short red HB, while the reddening-sensitive optical-IR CMDs in the bottom row of Fig. 18 reveal significant differential reddening across the target field.

\subsection{Terzan $9=$ Gcl-80.1}

The first optical CMD was presented by Ortolani et al. (1999b), who estimate $E(B-V)=1.95$ and $R_{\odot}=4.9 \pm 0.7$ kpc via direct comparison to the GGC M 30. Using near-IR photometry, Valenti et al. (2010) use the measured RGB slope and tip to obtain $E(B-V)=1.79$ and $R_{\odot}=5.6 \mathrm{kpc}$. The only spectroscopic metallicity estimate remains the CaII triplet study of Armandroff \& Zinn (1988), giving $[\mathrm{Fe} / \mathrm{H}]=-0.99$. Using a second epoch of optical imaging, Rossi et al. (2015) mention that this cluster has a relatively high proper motion compared to other bulge GGCs. Our CMDs in Fig. 19 suggest that this cluster is both fairly sparse and severely affected by differential reddening, so any morphological conclusions must be postponed to a future study presenting differential reddening corrected CMDs.

\subsection{Terzan $10=$ ESO 521-16}

An optical CMD was presented by Ortolani et al. (1997a), but they find that the cluster HB is at the detection limit of their data. They note the large differential reddening towards this cluster and estimate $R_{\odot}=4.8 \pm 1.0 \mathrm{kpc}$. This value was substantially revised by Alonso-García et al. (2015) using near-IR time series imaging from VVV, and by exploiting the differential reddening among cluster RRL, they find an unusually flat near-IR extinction law of $A_{K s} / E\left(J-K_{S}\right)=0.47 \pm 0.05$, yielding a distance of $R_{\odot}=10.3 \pm 0.2 \pm 0.2 \mathrm{kpc}$, which places Terzan 10 on the opposite (far) side of the Galactic bulge from previous studies. This is another cluster with a low concentration parameter according to Bonatto \& Bica (2008b), who report $c=0.8 \pm 0.2$ or $0.7 \pm 0.3$ based on King fits to 2MASS density profiles and surface brightness profiles respectively. Despite our smaller $\left(\sim 200^{\prime \prime}\right.$ per side) field of view as compared to Alonso-García et al. (2015), our photometry also illustrates severe differential reddening across the target field of view.

\subsection{Terzan $12=$ ESO 522-1}

The optical CMD presented by Ortolani et al. (1998) is the only published one for this cluster, and they use the HB to calculate $E(B-V)=2.06 \pm 0.06$ and $R_{\odot}=3.4 \pm 0.5 \mathrm{kpc}$. Based on high resolution optical spectroscopy of three stars, Côté (1999) list $[\mathrm{Fe} / \mathrm{H}]=-0.5$ and a radial velocity of $94.1 \pm 1.5 \mathrm{~km} / \mathrm{s}$. Our photometry in Fig. 21 shows significant differential reddening, and also a likely red HB.

$$
\text { 4.16. Ton 2 }=\text { Pismis } 26=E S O 333-16
$$

An optical VI CMD was presented by Bica et al. (1996), who use a direct comparison with 47 Tuc to estimate $E(B-V)=1.26$ and $R_{\odot}=6.4 \pm 0.6 \mathrm{kpc}$. The only spectroscopic abundance value is from Côté (1999), who finds $[\mathrm{Fe} / \mathrm{H}]=-0.6$ and a radial velocity of $-184.4 \pm 2.2 \mathrm{~km} / \mathrm{s}$ from optical echelle spectra of three stars. The cluster CMDs in Fig. 22 reveal low field contamination and a clear red HB. However, the shape of the red HB in the optical-IR CMDs clarifies that despite the clear definition of the cluster sequences in this case, differential reddening persists over the cluster field.

\section{SUMMARY}

We have presented the observation and data reduction strategy for multi-wavelength space-based imaging of 16 GGCs located towards the inner Galactic bulge and disk. Even a cursory glance at the cluster and parallel field 
CMDs (uncorrected for differential reddening) indicates the diversity of cluster properties (metallicity, density, HB morphology) sampled by our target clusters. However, these CMDs also reveal a remarkable variation in the distribution of bulge and disk populations along the various sightlines probed by our imaging, useful in their own right to constrain models of Galactic structure and extinction towards the inner Milky Way. Forthcoming studies will exploit this imaging to quantitatively address and refine existing values (based largely on optical imaging) of cluster structural parameters, distances and HB morphology, using differential reddening maps to optimize our photometry, while measuring target cluster ages, mass functions and mass segregation for the first time. Lastly, due to its spatial resolution, our imaging comprises a first epoch enabling future proper motion studies of our target clusters as well as their foreground and background disk and bulge components.

The HST imaging used in our analysis is available at [10.17909/T9HD59].

It is a pleasure to thank the anonymous referee for their valuable comments and suggestions. R.E.C. acknowledges support from Gemini-CONICYT for Project 32140007. J.A-G. acknowledges support by FONDECYT Iniciación 11150916, MINEDUC ANT 1655 and by the Ministry of Economy, Development, and Tourisms Millennium Science Initiative through grant IC120009, awarded to the Millennium Institute of Astrophysics (MAS). M.H. acknowledges support from BASAL CATA Center for Astrophysics and Associated Technologies PFB-06. D.G. and F.M. also acknowledge financial support from the Chilean BASAL Centro de Excelencia en Astrofisica y Technologias Afines (CATA) grant PFB-06/2007. This publication makes use of observations collected at the European Organization for Astronomical Research in the Southern Hemisphere, Chile, under ESO program 179.B-2002 (VVV survey), as well as the SIMBAD databased, operated at CDS, Strasbourg, France.

\section{Facilities: HST (ACS,WFC3), ESO:VISTA}

\section{REFERENCES}

Alcaino, G., \& Liller, W. 1983, AJ, 88, 1166

Alonso-García, J., Mateo, M., Sen, B., et al. 2012, AJ, 143, 70

Alonso-García, J., Dékány, I., Catelan, M., et al. 2015, AJ, 149,99

Altamirano, D., van der Klis, M., Méndez, M., et al. 2008, ApJ, 687, 488-504

Anderson, J., \& van der Marel, R. P. 2010, ApJ, 710, 1032

Armandroff, T. E., \& Zinn, R. 1988, AJ, 96, 92

Bahramian, A., Heinke, C. O., Sivakoff, G. R., et al. 2016, The Astronomer's Telegram, 9072, 1

Barbuy, B., Ortolani, S., \& Bica, E. 1997, A\&AS, 122, 483

Barbuy, B., Zoccali, M., Ortolani, S., et al. 2006, A\&A, 449, 349

Barbuy, B., Cantelli, E., Vemado, A., et al. 2016, A\&A, 591, A53

Barret, D., Mereghetti, S., Roques, J. P., et al. 1991, ApJL, 379, L21

Barret, D., Grindlay, J. E., Harrus, I. M., \& Olive, J. F. 1999, A\&A, 341, 789

Bellini, A., Anderson, J., van der Marel, R. P., et al. 2014, ApJ, 797, 115

Bica, E., Ortolani, S., \& Barbuy, B. 1994, A\&A, 283, 67

Bica, E., Ortolani, S., \& Barbuy, B. 1996, A\&AS, 120, 153

Bica, E., Ortolani, S., \& Barbuy, B. 2016, PASA, 33, e028
Bohlin, R. C. 2012, Instrument Science Report ACS 2012-01

Bonatto, C., \& Bica, E. 2008a, A\&A, 477, 829

Bonatto, C., \& Bica, E. 2008b, A\&A, 479, 741

Bonatto, C., Bica, E., Ortolani, S., \& Barbuy, B. 2009, MNRAS, 397, 1032

Borrel, V., Bouchet, L., Jourdain, E., et al. 1996, A\&AS, 120,249

Brown, T. M., Sahu, K., Anderson, J., et al. 2010, ApJL, 725, L19

Cackett, E. M., Wijnands, R., Heinke, C. O., et al. 2006, MNRAS, 369, 407

Carballo-Bello, J. A., Ramírez Alegría, S., Borissova, J., et al. 2016, MNRAS, 462, 501

Carretta, E., Bragaglia, A., Gratton, R., D’Orazi, V., \& Lucatello, S. 2009, A\&A, 508, 695

Christian, C. A., \& Friel, E. D. 1992, AJ, 103, 142

Cohen, R. E., \& Sarajedini, A. 2012, MNRAS, 419, 342

Cohen, R. E., Mauro, F., Geisler, D., et al. 2014, AJ, 148, 18

Cohen, R. E., Hempel, M., Mauro, F., et al. 2015, AJ, 150, 176

Cohen, R. E., Moni Bidin, C., Mauro, F., Bonatto, C., \& Geisler, D. 2017, MNRAS, 464, 1874

Correnti, M., Gennaro, M., Kalirai, J. S., Brown, T. M., \& Calamida, A. 2016, ApJ, 823, 18 


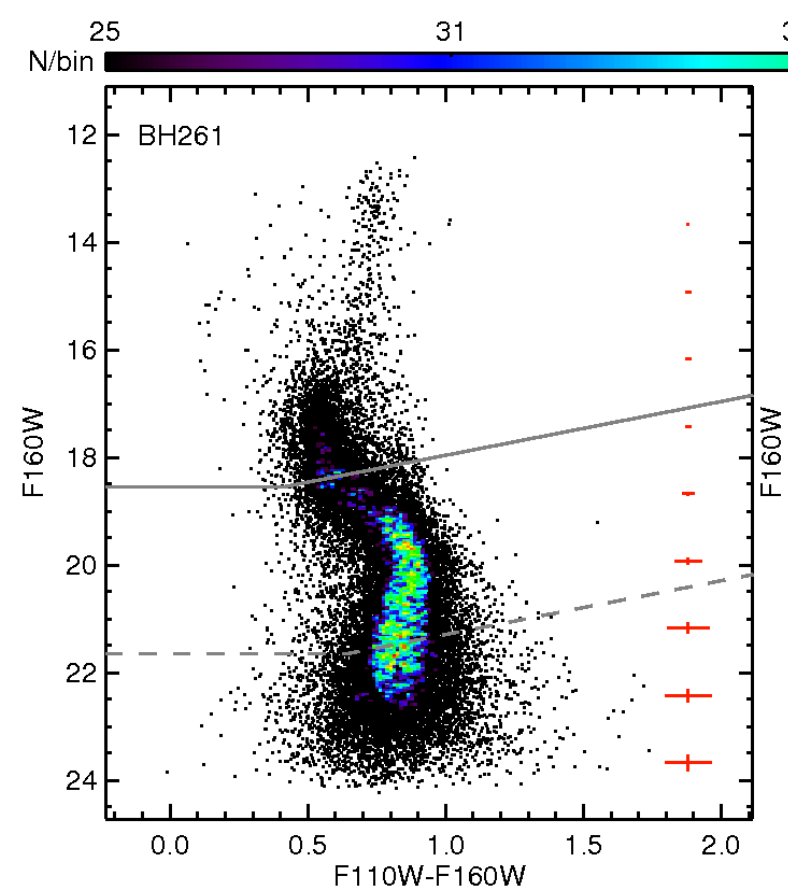

$38 \quad 46 \quad 57$
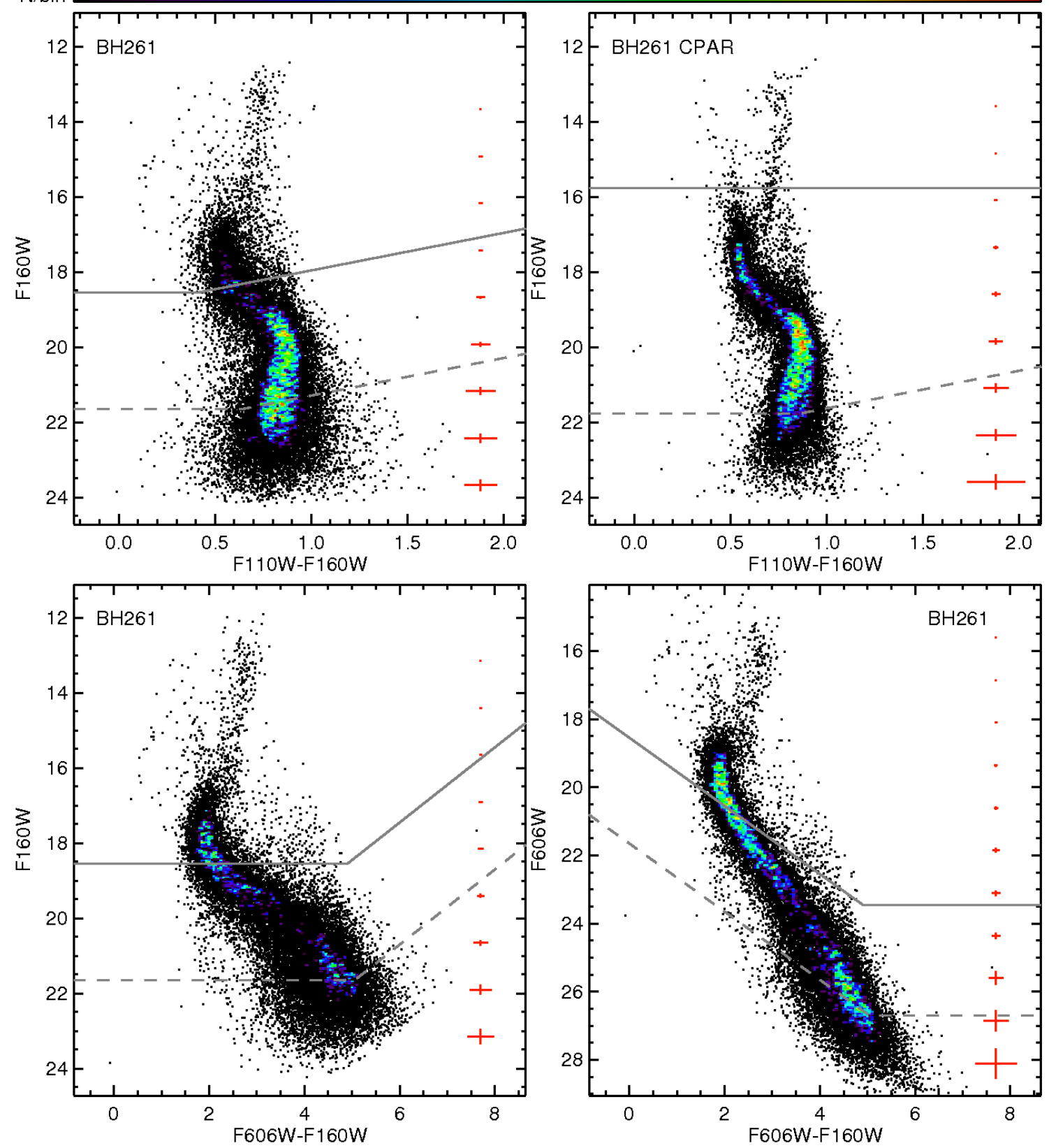

Figure 7. CMDs of the target cluster BH261 in several color-magnitude planes, including the parallel field in the upper right panel. The cluster CMDs in the other three panels are constructed from simultaneous photometry of all primary field images, showing all stars passing our quality cuts described in Sect. 3.3. CMDs are color-coded according to the logarithmic color scale shown in the colorbar at the top, and median photometric errors resulting from artificial star tests are shown in red along the right hand side of each CMD. The $50 \%$ and $90 \%$ radially integrated faint completeness limits are indicated by dotted and solid grey lines respectively. The color and magnitude scales of both CMD axes in each panel are kept fixed in all subsequent figures to enable direct comparisons between clusters. 


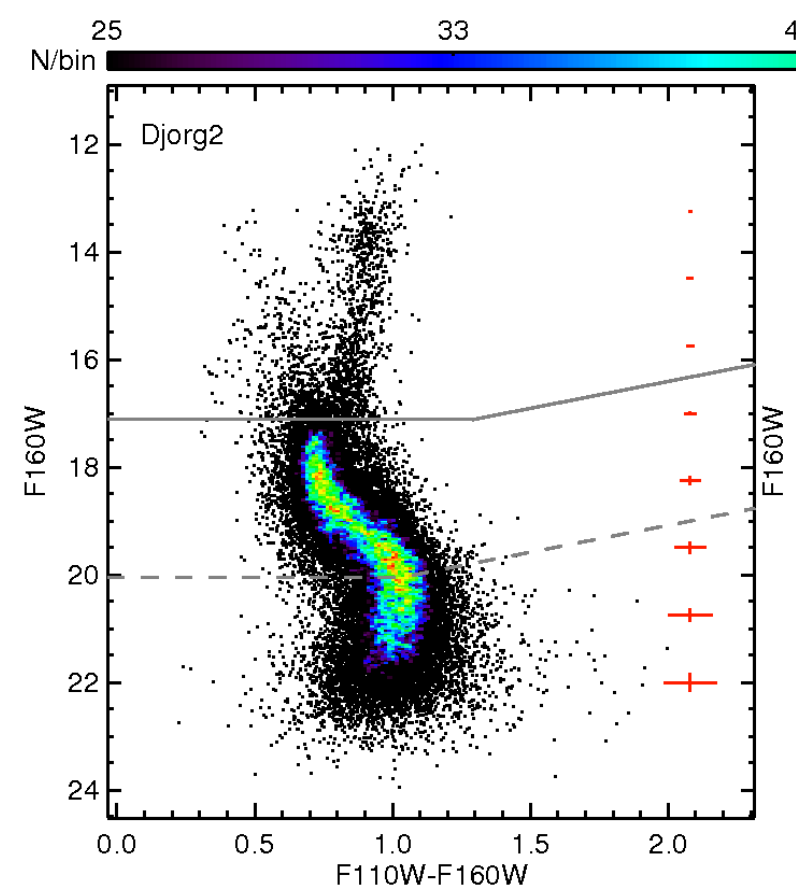

$43 \quad 56 \quad 74$
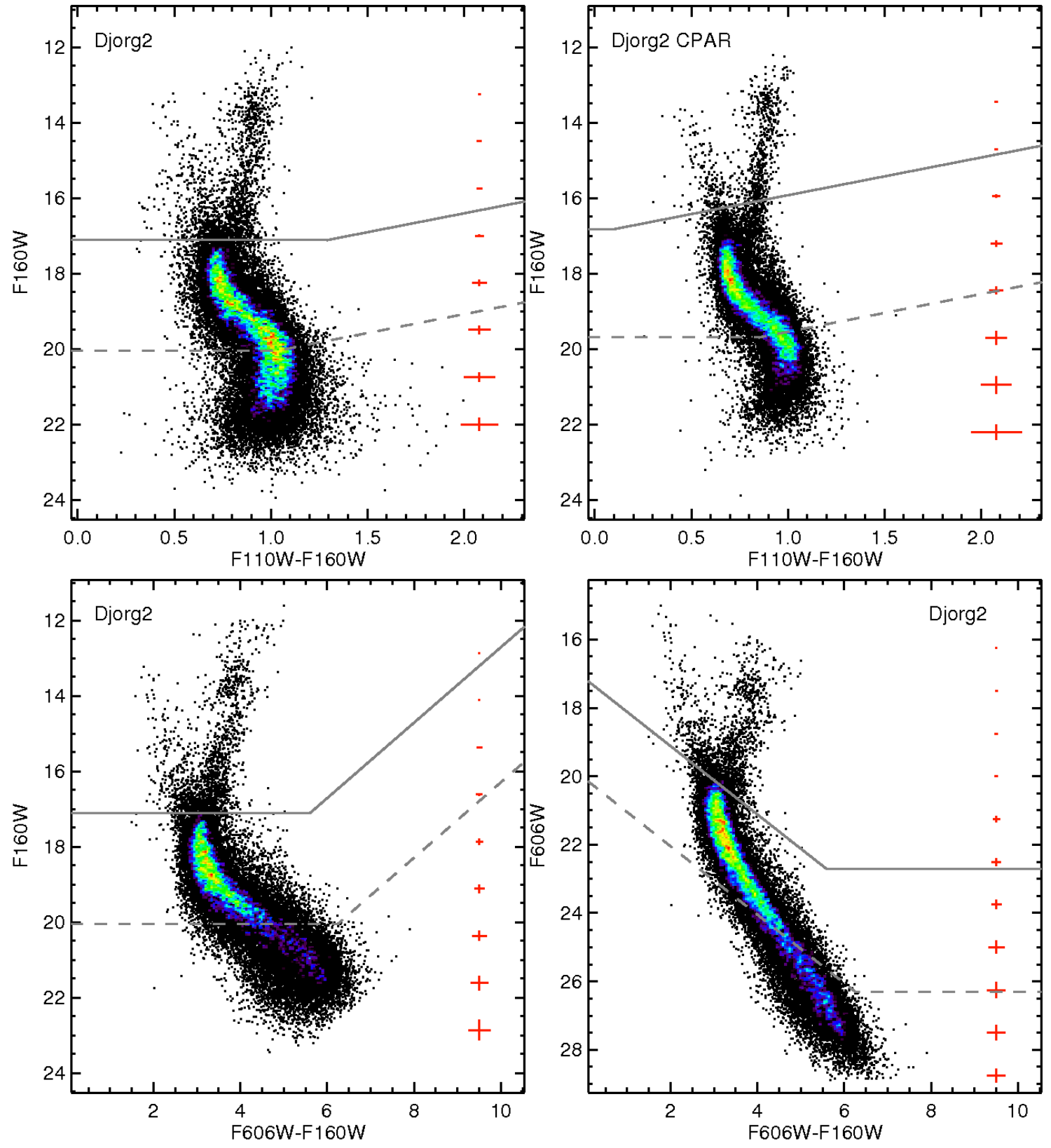

Figure 8. As in Fig. 7, but for Djorg 2. 


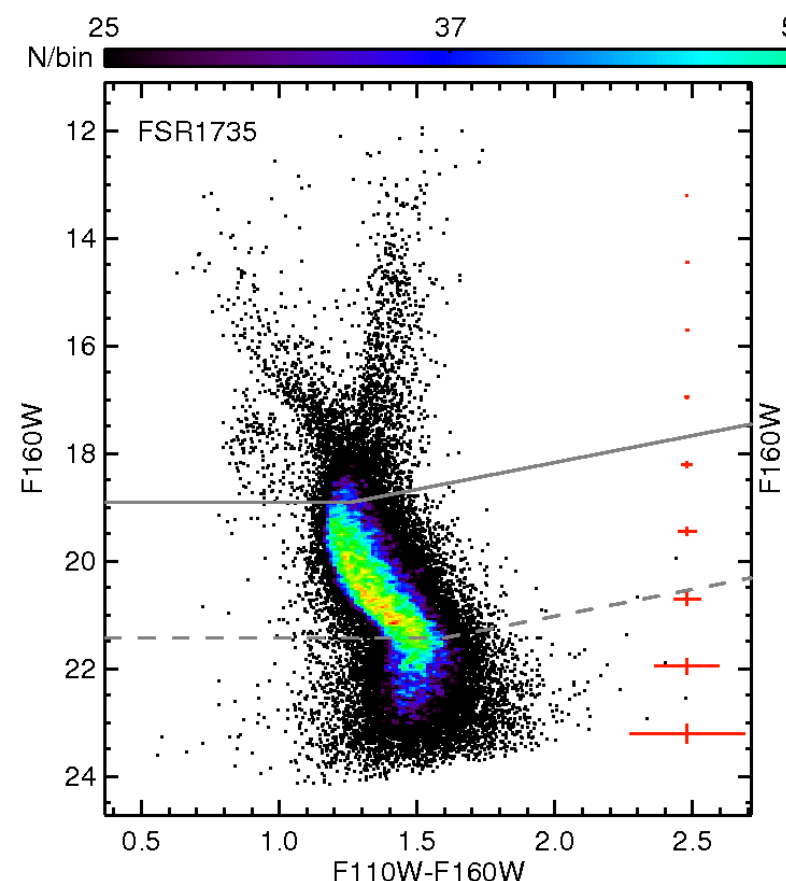

55

82

122
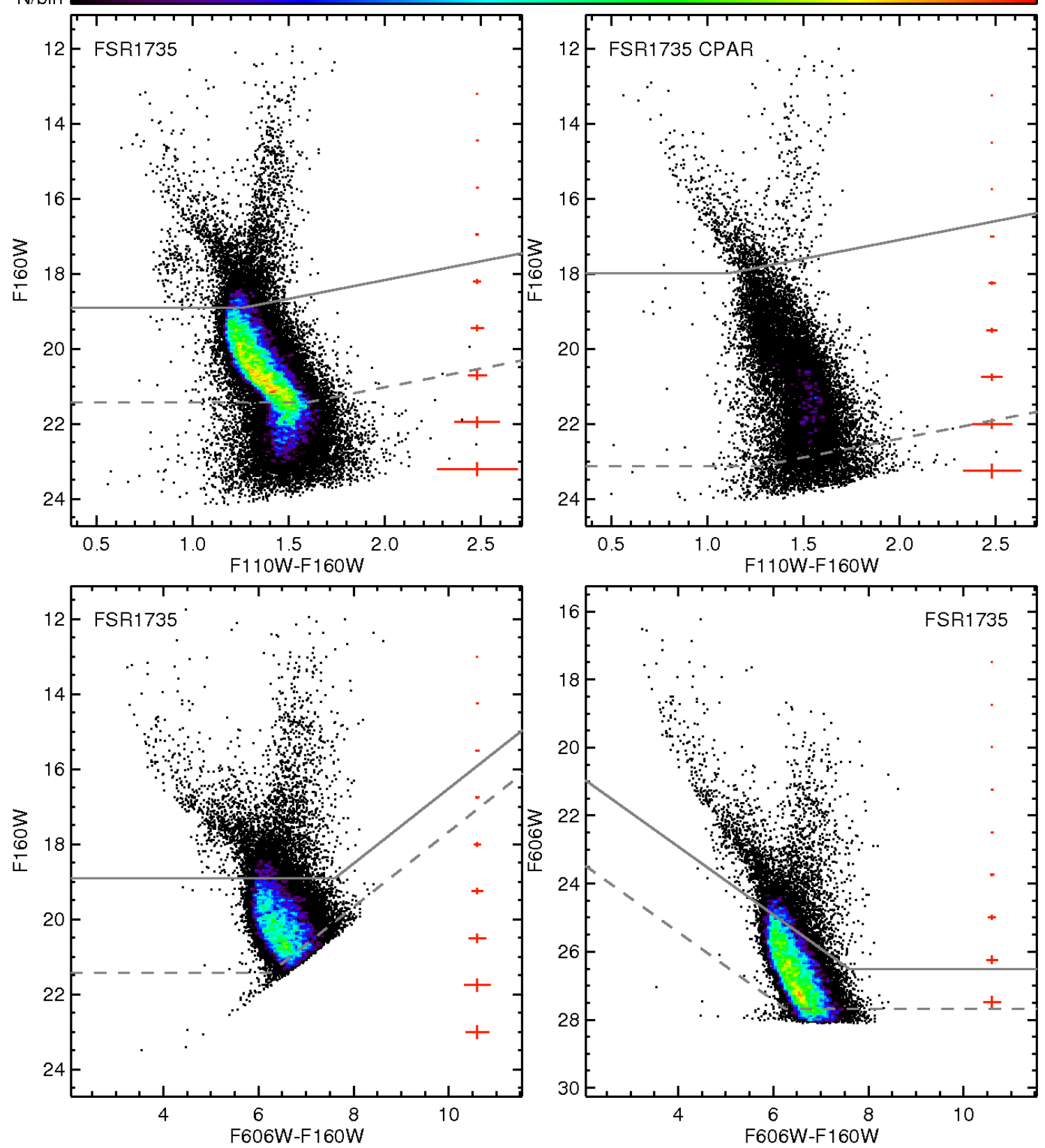

Figure 9. As in Fig. 7, but for FSR 1735.

Côté, P. 1999, AJ, 118, 406

Dalessandro, E., Saracino, S., Origlia, L., et al. 2016, ApJ, 833,111

Davidge, T. J. 2000, AJ, 120, 1853

De Angeli, F., Piotto, G., Cassisi, S., et al. 2005, AJ, 130, 116

Dias, B., Barbuy, B., Saviane, I., et al. 2016, A\&A, 590, A9 Dieball, A., Bedin, L. R., Knigge, C., et al. 2016, ApJ, 817, 48

Dolphin, A. E. 2000, PASP, 112, 1383
Dotter, A., Sarajedini, A., Anderson, J., et al. 2010, ApJ, 708, 698

Dotter, A., Sarajedini, A., \& Anderson, J. 2011, ApJ, 738, 74

Dotter, A., Ferguson, J. W., Conroy, C., et al. 2015, MNRAS, 446, 1641

Dressel, L. 2018, "Wide Field Camera 3 Instrument Handbook, Version 10.0" (Baltimore:STScI)

Fahlman, G. G., Douglas, K. A., \& Thompson, I. B. 1995, AJ, 110, 2189 


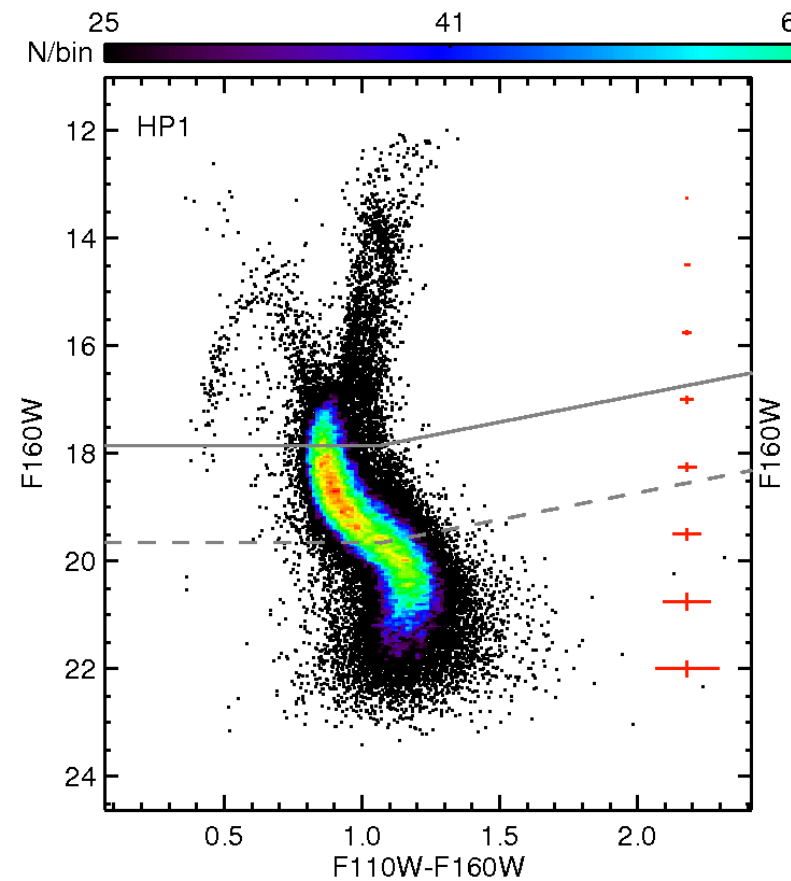

68

113

186
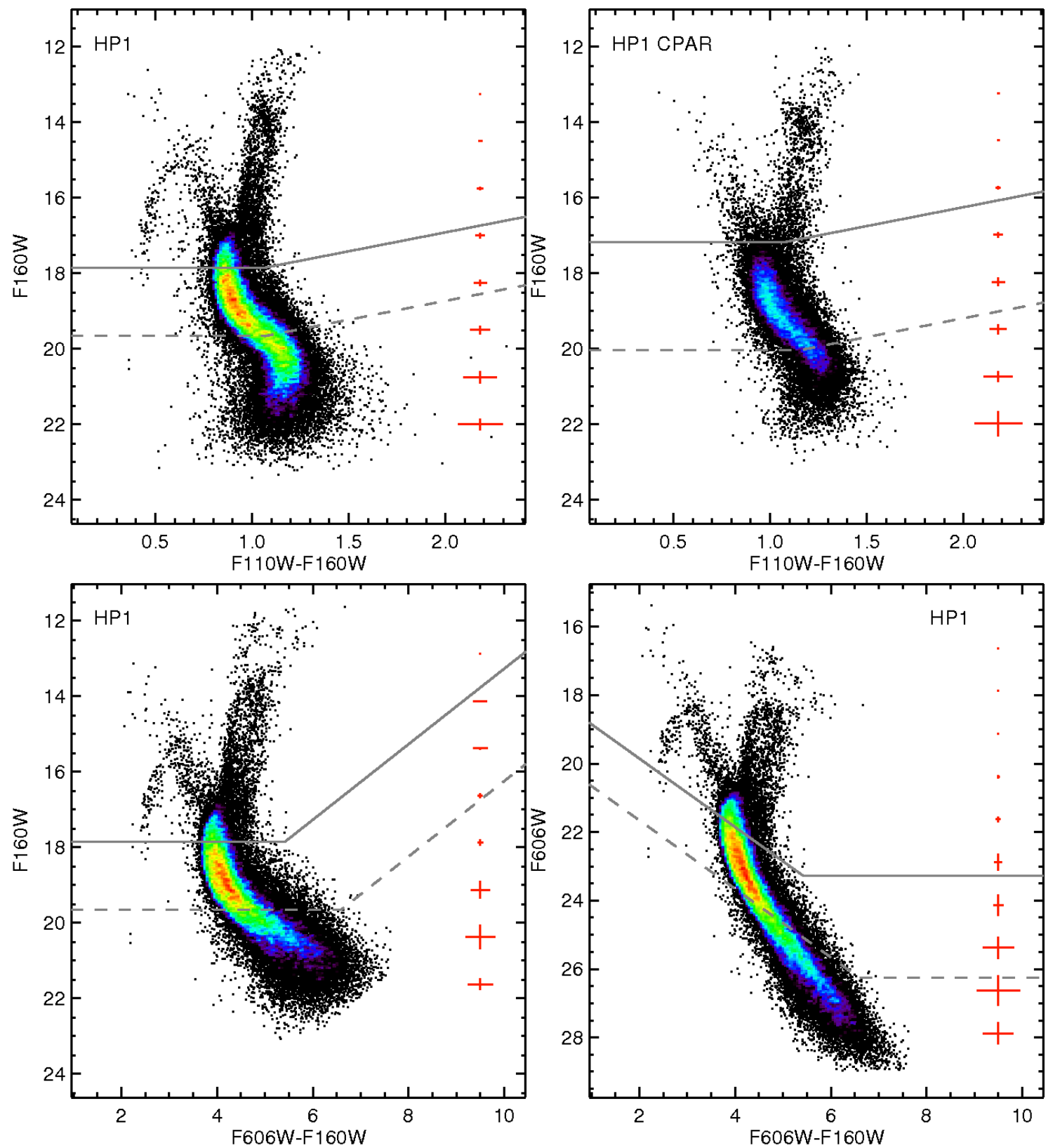

Figure 10. As in Fig. 7, but for HP 1.

Fernández-Trincado, J. G., Zamora, O., Souto, D., et al. 2018, arXiv:1801.07136

Ferraro, F. R., Valenti, E., \& Origlia, L. 2006, ApJ, 649, 243

Ferraro, F. R., Dalessandro, E., Mucciarelli, A., et al. 2009, Nature, 462, 483

Ferraro, F. R., Massari, D., Dalessandro, E., et al. 2016, ApJ, 828, 75

Fiorentino, G., Massari, D., McConnachie, A., et al. 2016, arXiv:1608.01457
Froebrich, D., Meusinger, H., \& Scholz, A. 2007, MNRAS, 377, L54

Goldsbury, R., Richer, H. B., Anderson, J., et al. 2010, AJ, $140,1830-1837$

Goldsbury, R., Heyl, J., \& Richer, H. 2013, ApJ, 778, 57

Gonzalez, O. A., Rejkuba, M., Zoccali, M., et al. 2012, A\&A, 543, A13

Guainazzi, M., Parmar, A. N., Segreto, A., et al. 1998, A\&A, 339, 802 


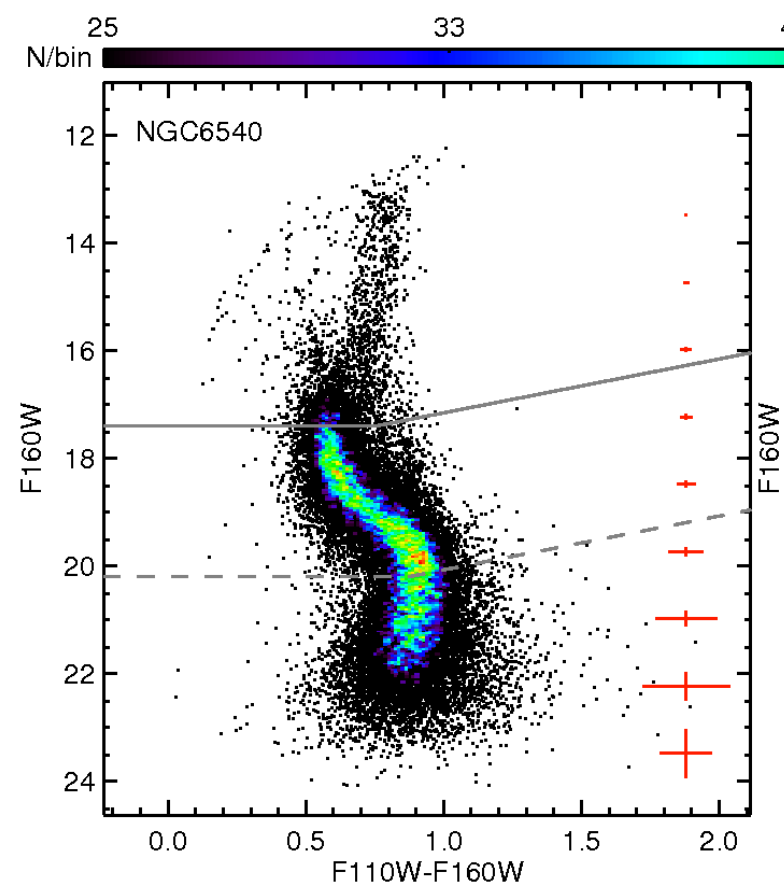

43

57
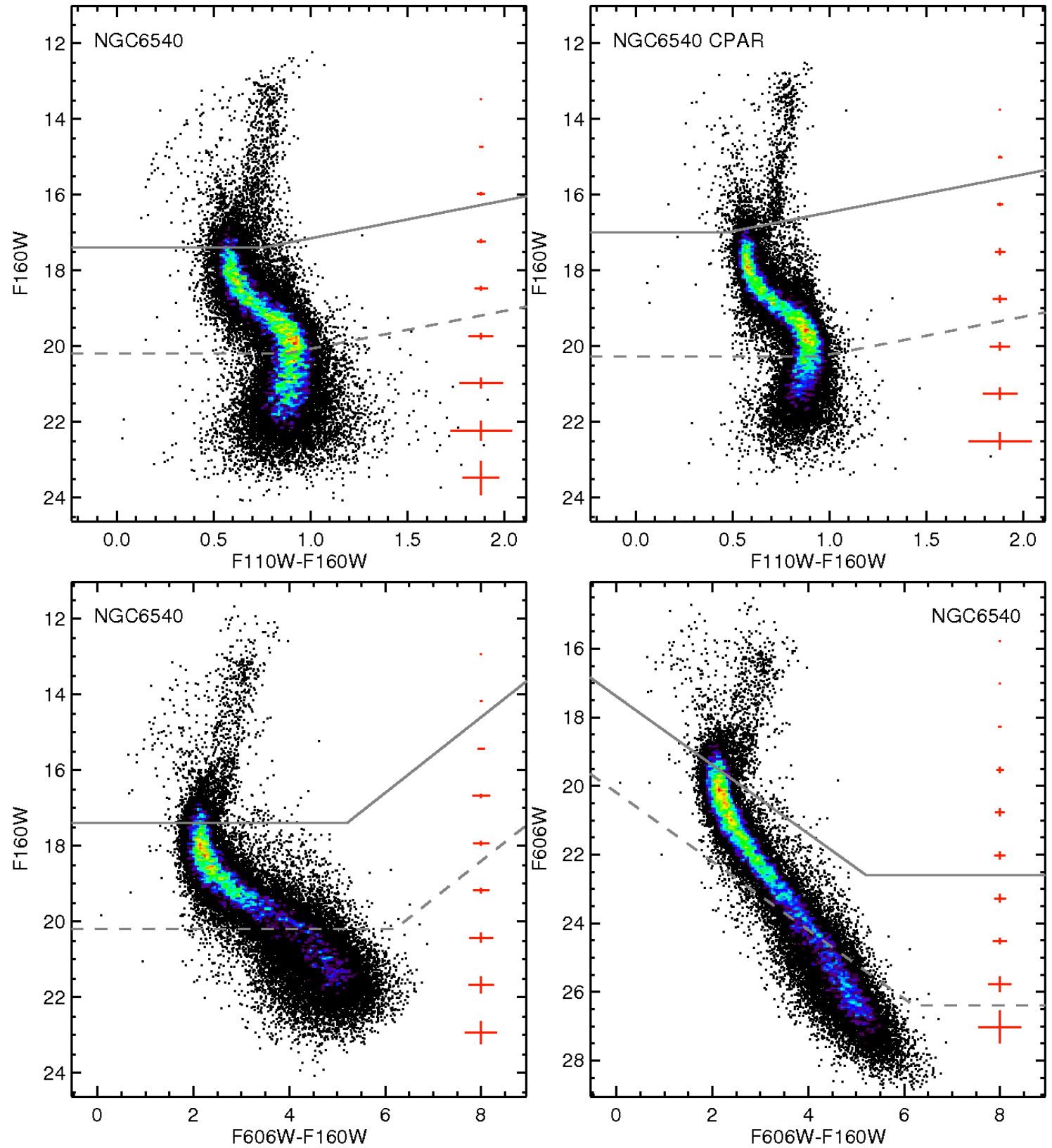

Figure 11. As in Fig. 7, but for NGC 6540.

Guainazzi, M., Parmar, A. N., \& Oosterbroek, T. 1999, A\&A, 349, 819

Harris, W. E. 1996, AJ, 112, 1487

Hesser, J. E., Harris, H. C., \& Harris, G. L. H. 1984, BAAS, 16, 967

Idiart, T. P., Barbuy, B., Perrin, M.-N., et al. 2002, A\&A, 381,472

in't Zand, J. J. M., Hulleman, F., Markwardt, C. B., et al. 2003, A\&A, 406, 233
Johnson, C. I., Rich, R. M., Caldwell, N., et al. 2018, AJ, 155,71

Johnston, H. M., Verbunt, F., \& Hasinger, G. 1995, A\&A, 298, L21

Kerber, L. O., Nardiello, D., Ortolani, S., et al. 2018, ApJ, 853,15

King, I. 1962, AJ, 67, 471 


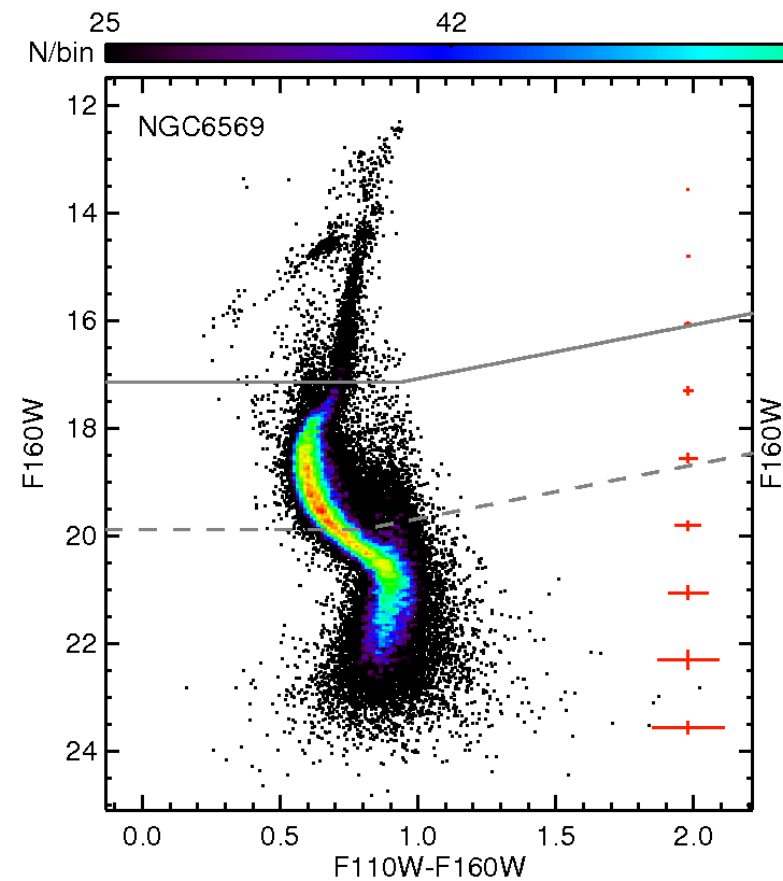

$71 \quad 119 \quad 200$
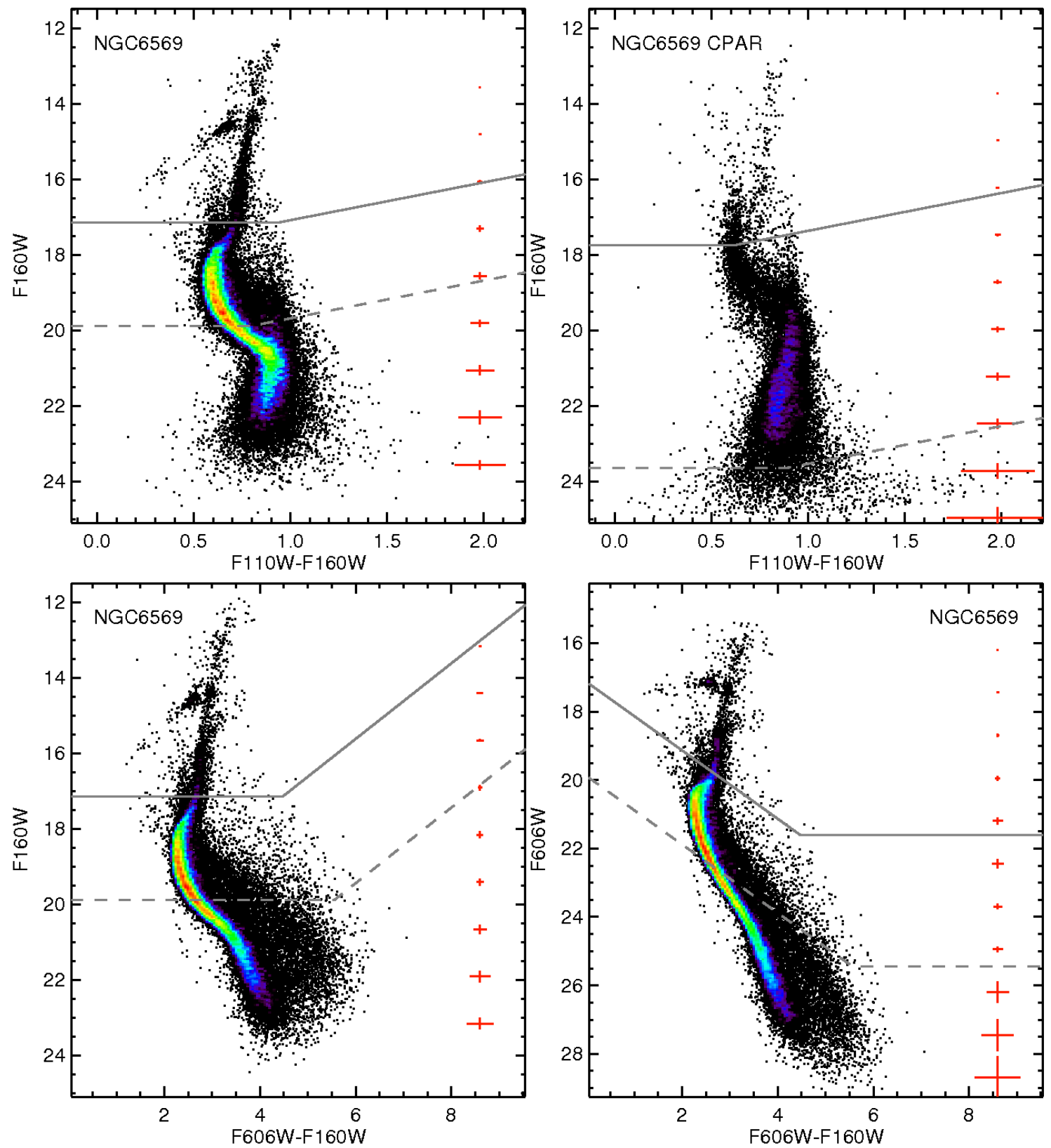

Figure 12. As in Fig. 7, but for NGC 6569.

Kunder, A., Stetson, P. B., Catelan, M., et al. 2015, Fifty

Years of Wide Field Studies in the Southern Hemisphere:

Resolved Stellar Populations of the Galactic Bulge and

Magellanic Clouds, 491, 104

Lagioia, E. P., Milone, A. P., Stetson, P. B., et al. 2014, ApJ, 785, 81

Lanzoni, B., Ferraro, F. R., Dalessandro, E., et al. 2010, ApJ, 717, 653

Leaman, R., VandenBerg, D. A., \& Mendel, J. T. 2013, MNRAS, 436, 122
Lee, J.-W., \& Carney, B. W. 2002, AJ, 123, 3305

Lee, J.-W., Carney, B. W., \& Balachandran, S. C. 2004, AJ, 128, 2388

Makishima, K., Ohashi, T., Inoue, H., et al. 1981, ApJL, 247, L23

Marín-Franch, A., Aparicio, A., Piotto, G., et al. 2009, ApJ, 694, 1498

Mauro, F. et al., 2012, ApJL, 761, 29

Mauro, F., Moni Bidin, C., Chené, A.-N. et al., 2013, RMxAA, 49, 189 

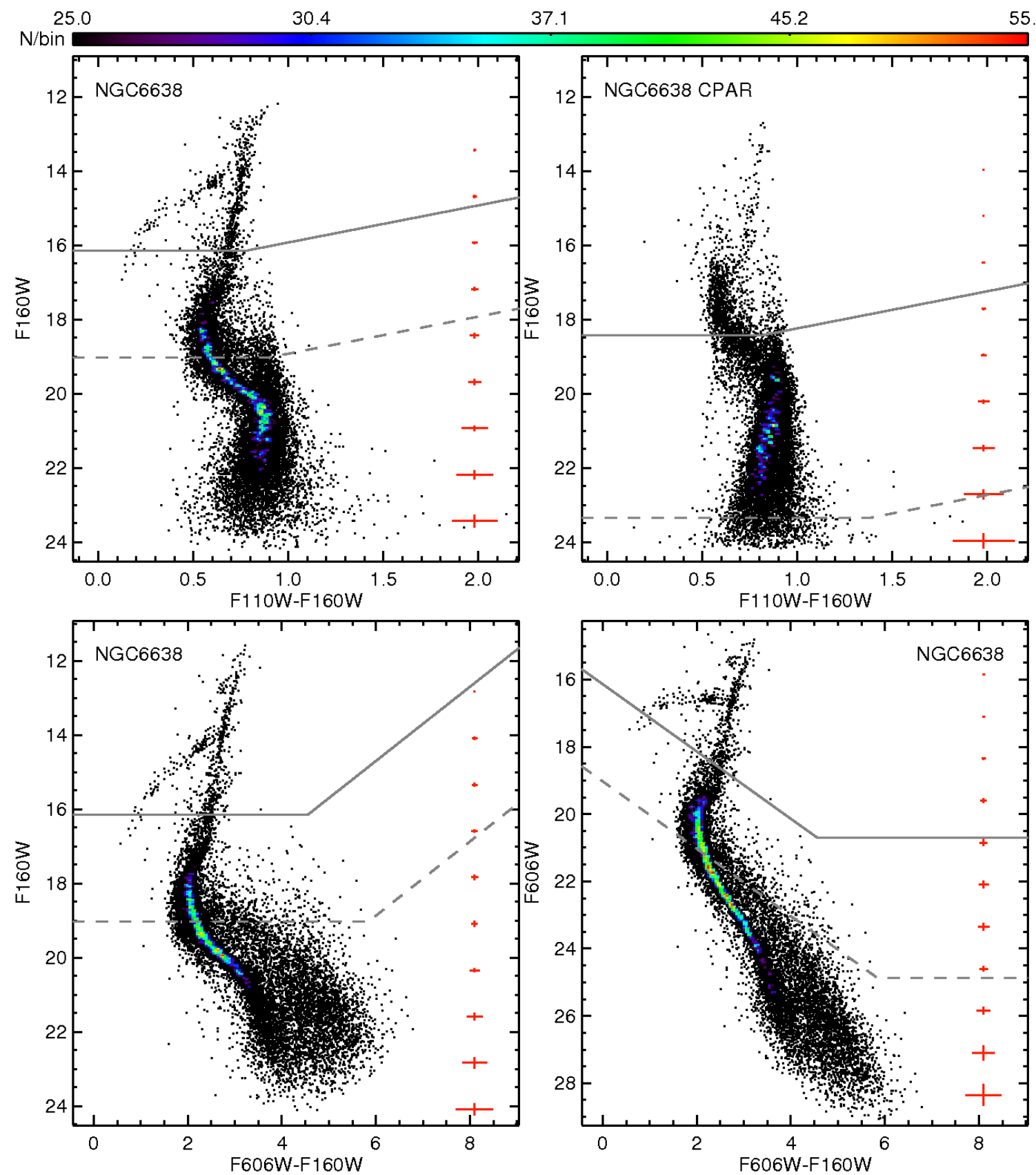

Figure 13. As in Fig. 7, but for NGC 6638

Mauro, F., Moni Bidin, C., Geisler, D., et al. 2014, A\&A, 563, A76

Massari, D., Mucciarelli, A., Ferraro, F. R., et al. 2014, ApJ, 795, 22

Massari, D., Fiorentino, G., McConnachie, A., et al. 2016, A\&A, 586, A51

McLaughlin, D. E. \& van der Marel, R. P., 2005, ApJS, 161, 304

Mereghetti, S., Barret, D., Stella, L., et al. 1995, A\&A, 302, 713
Milone, A. P., Bedin, L. R., Piotto, G., et al. 2008, ApJ, 673, 241-250

Milone, A. P., Piotto, G., Bedin, L. R., et al. 2012, A\&A, 540, A16

Milone, A. P., Marino, A. F., Piotto, G., et al. 2015, ApJ, 808,51

Minniti, D., Olszewski, E. W., \& Rieke, M. 1995, AJ, 110, 1686

Minniti, D. 1995, A\&A, 303, 468 


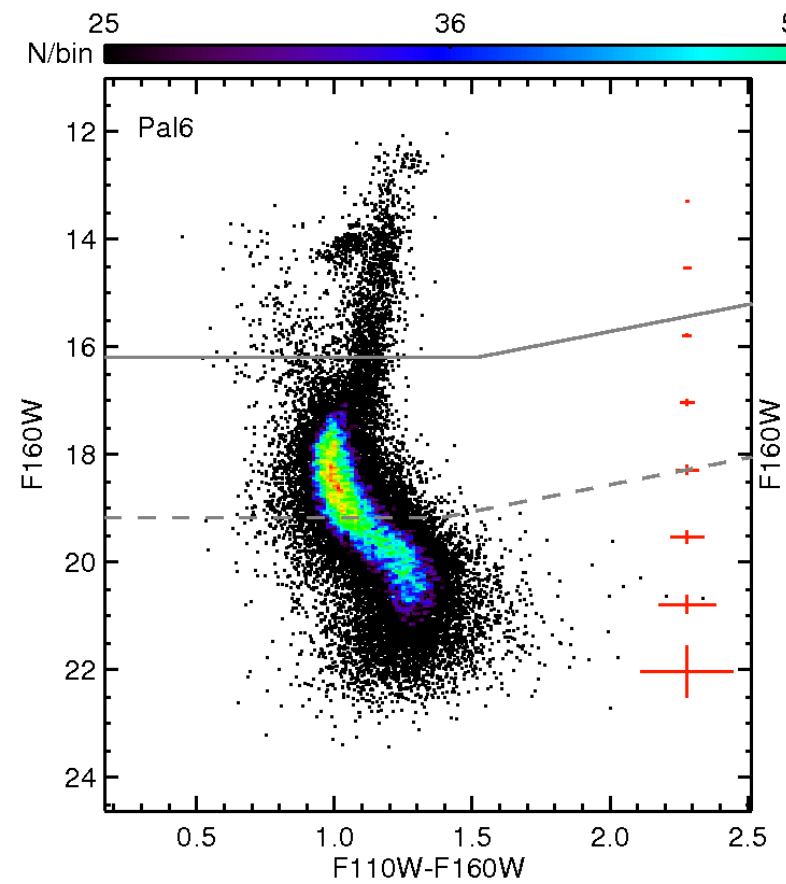

52

76
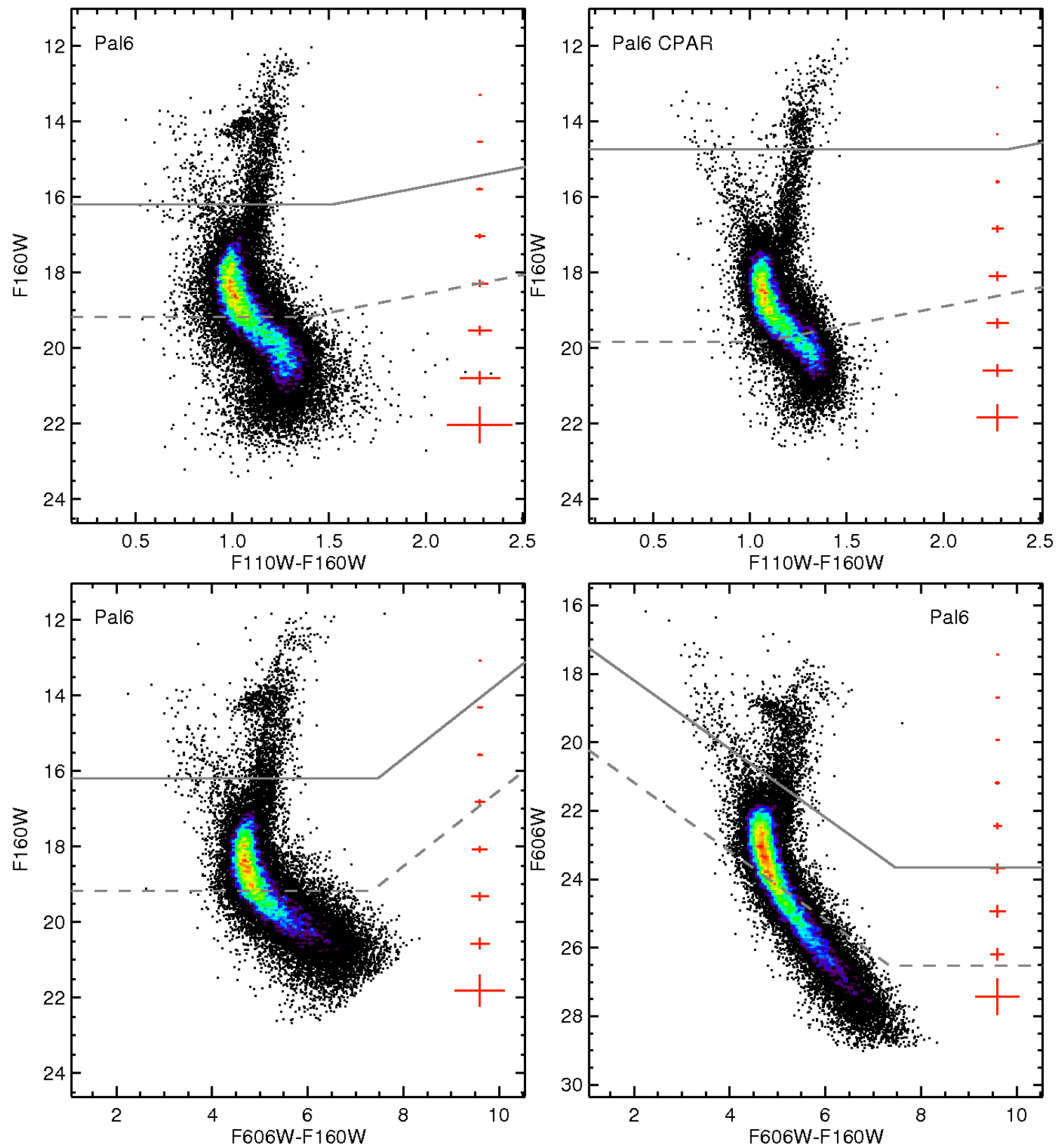

Figure 14. As in Fig. 7, but for Palomar 6.

Minniti, D., Lucas, P. W., Emerson, J. P., et al. 2010, NewA, 15, 433

Minniti, D., Hempel, M., Toledo, I., et al. 2011, A\&A, 527, A81

Minniti, D., Palma, T., Dékány, I., et al. 2017, arXiv:1703.02033

Miocchi, P., Lanzoni, B., Ferraro, F. R., et al. 2013, ApJ, 774,151

Molkov, S. V., Grebenev, S. A., \& Lutovinov, A. A. 2001, Astronomy Letters, 27, 363
Muñoz, C., Villanova, S., Geisler, D., et al. 2017, A\&A, submitted

Ness, M., Zasowski, G., Johnson, J. A., et al. 2016, ApJ, 819,2

Noyola, E., \& Gebhardt, K. 2006, AJ, 132, 447

O'Malley, E. M., Gilligan, C., \& Chaboyer, B. 2017, arXiv:1703.01915

Origlia, L., \& Rich, R. M. 2004, AJ, 127, 3422

Origlia, L., Massari, D., Rich, R. M., et al. 2013, ApJL, 779, L5 


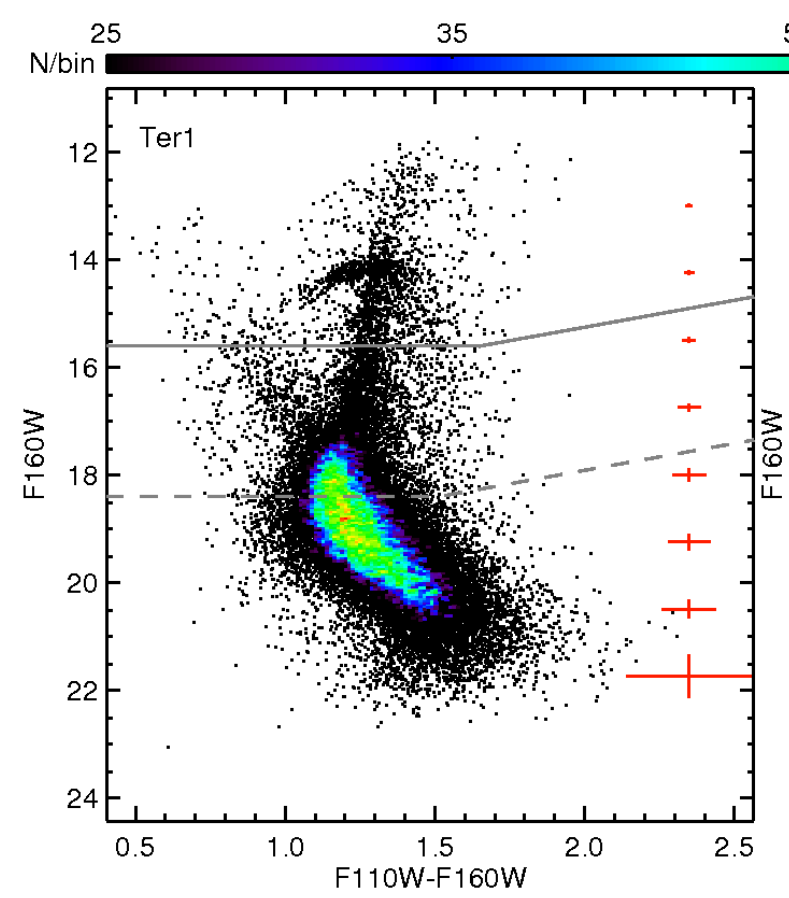

50

71
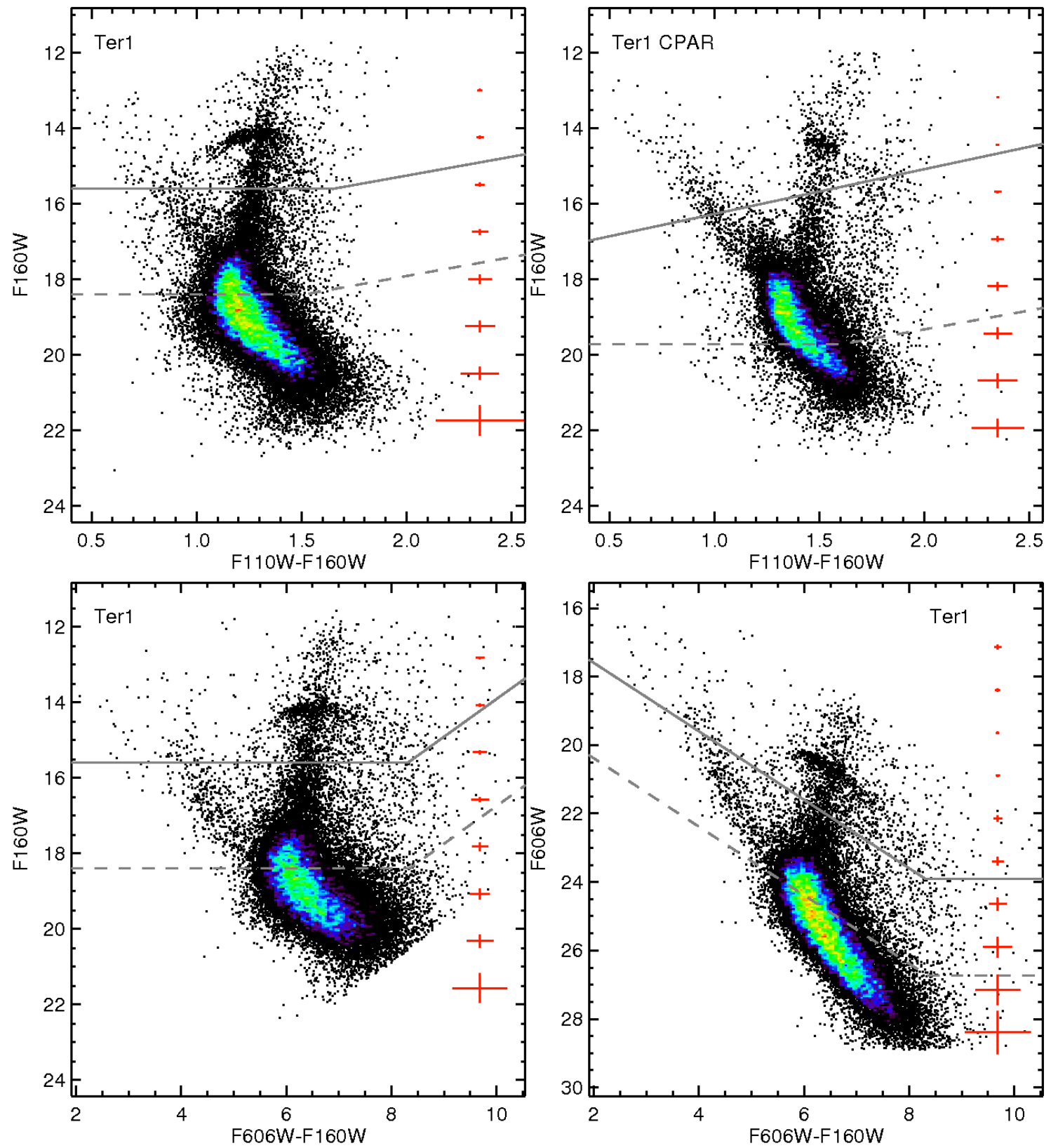

Figure 15. As in Fig. 7, but for Terzan 1.

Ortolani, S., Bica, E., \& Barbuy, B. 1993, A\&A, 267, 66

Ortolani, S., Bica, E., \& Barbuy, B. 1995, A\&A, 296, 680 Ortolani, S., Bica, E., \& Barbuy, B. 1997a, A\&AS, 126, 319 Ortolani, S., Bica, E., \& Barbuy, B. 1997b, MNRAS, 284, 692

Ortolani, S., Bica, E., \& Barbuy, B. 1997c, A\&A, 326, 614 Ortolani, S., Barbuy, B., \& Bica, E. 1997d, A\&A, 319, 850 Ortolani, S., Bica, E., \& Barbuy, B. 1998, A\&AS, 127, 471 Ortolani, S., Barbuy, B., Bica, E., et al. 1999a, A\&A, 350, 840
Ortolani, S., Bica, E., \& Barbuy, B. 1999b, A\&AS, 138, 267 Ortolani, S., Barbuy, B., Bica, E., et al. 2001a, A\&A, 376, 878

Ortolani, S., Bica, E., \& Barbuy, B. 2001b, A\&A, 374, 564

Ortolani, S., Bica, E., \& Barbuy, B. 2006, ApJL, 646, L115

Ortolani, S., Barbuy, B., Bica, E., Zoccali, M., \& Renzini, A. 2007, A\&A, 470, 1043

Ortolani, S., Barbuy, B., Momany, Y., et al. 2011, ApJ, 737,31 


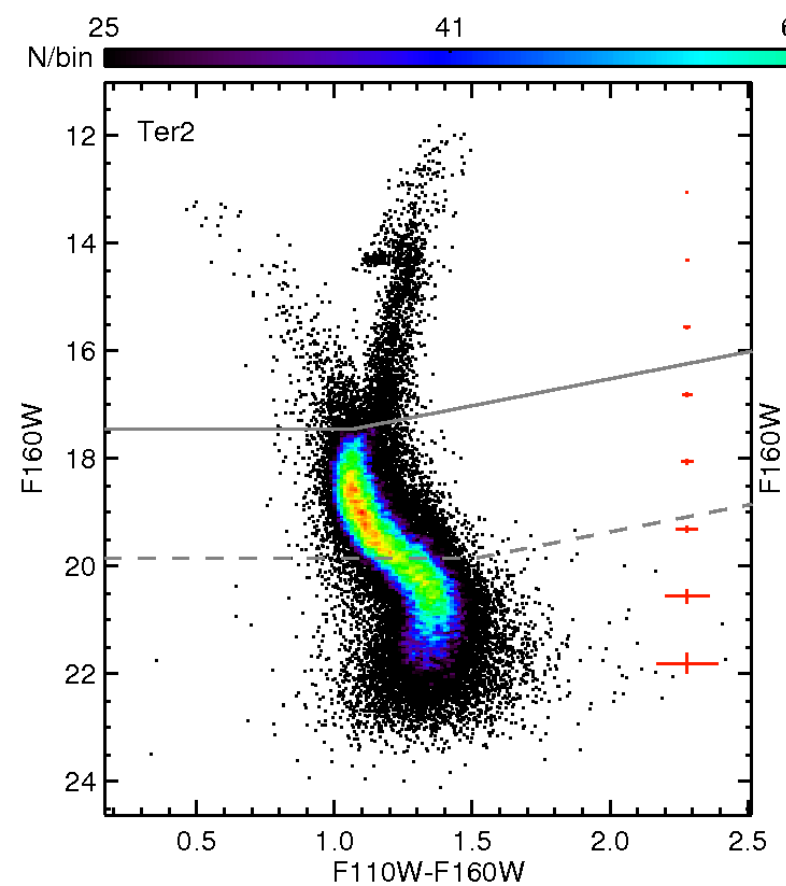

67

109

178
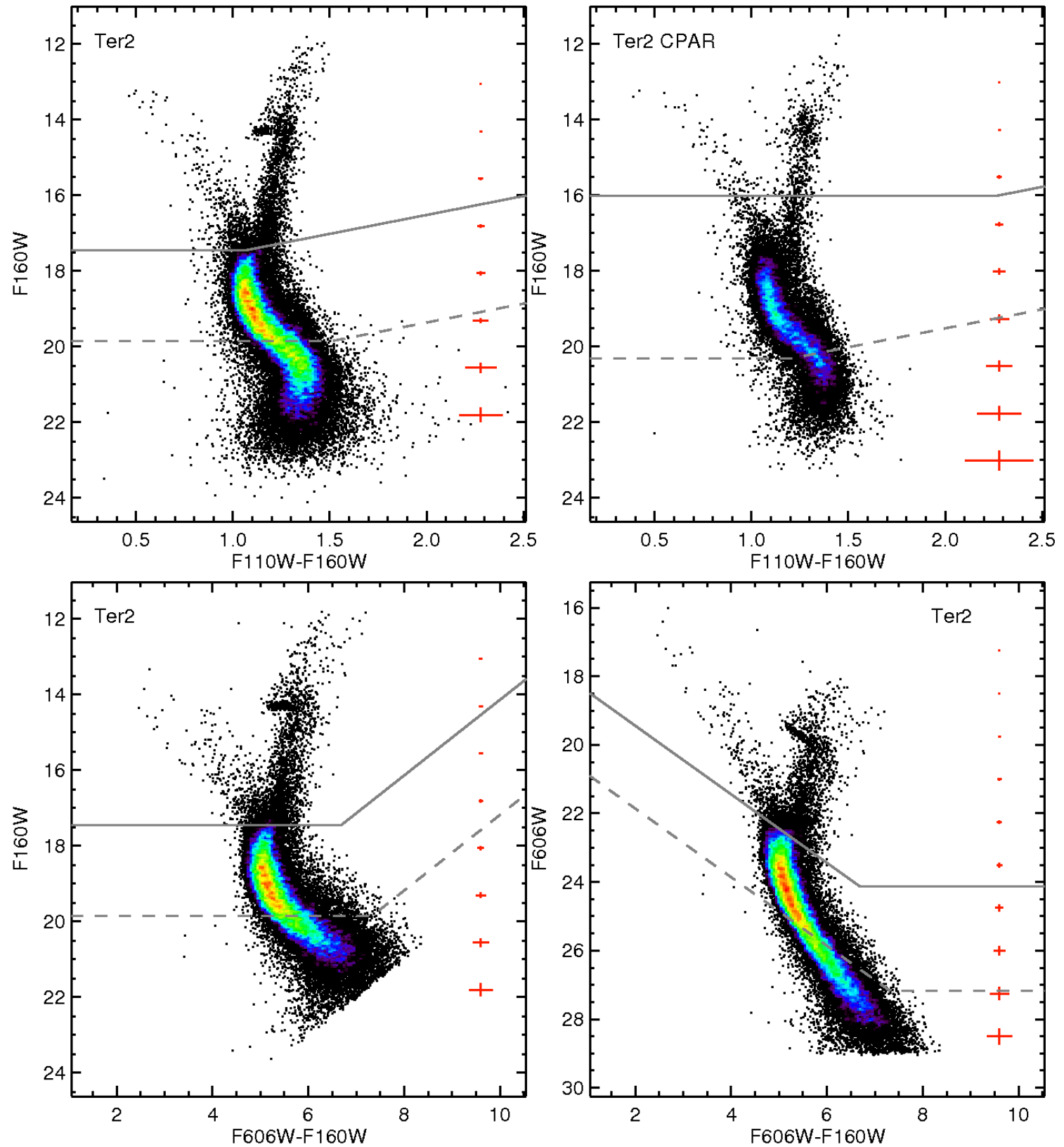

Figure 16. As in Fig. 7, but for Terzan 2.

Ortolani, S., Bonatto, C., Bica, E., Barbuy, B., \& Saito, R. K. 2012, AJ, 144, 147

Paust, N. E. Q., Reid, I. N., Piotto, G., et al. 2010, AJ, 139,476

Peñaloza, F., Pessev, P., Vaśquez, S., et al. 2015, PASP, 127,329

Piotto, G., King, I. R., Djorgovski, S. G., et al. 2002, A\&A, 391,945

Piotto, G., Milone, A. P., Bedin, L. R., et al. 2015, AJ, 149, 91
Ross, T. L., Holtzman, J. A., Anthony-Twarog, B. J., et al. 2014, AJ, 147, 4

Rossi, L. J., Ortolani, S., Barbuy, B., Bica, E., \& Bonfanti, A. 2015, MNRAS, 450, 3270

Rutily, B., \& Terzan, A. 1977, A\&AS, 30, 315

Samra, R. S., Richer, H. B., Heyl, J. S., et al. 2012, ApJL, 751, L12

Saracino, S., Dalessandro, E., Ferraro, F. R., et al. 2015, ApJ, 806, 152 


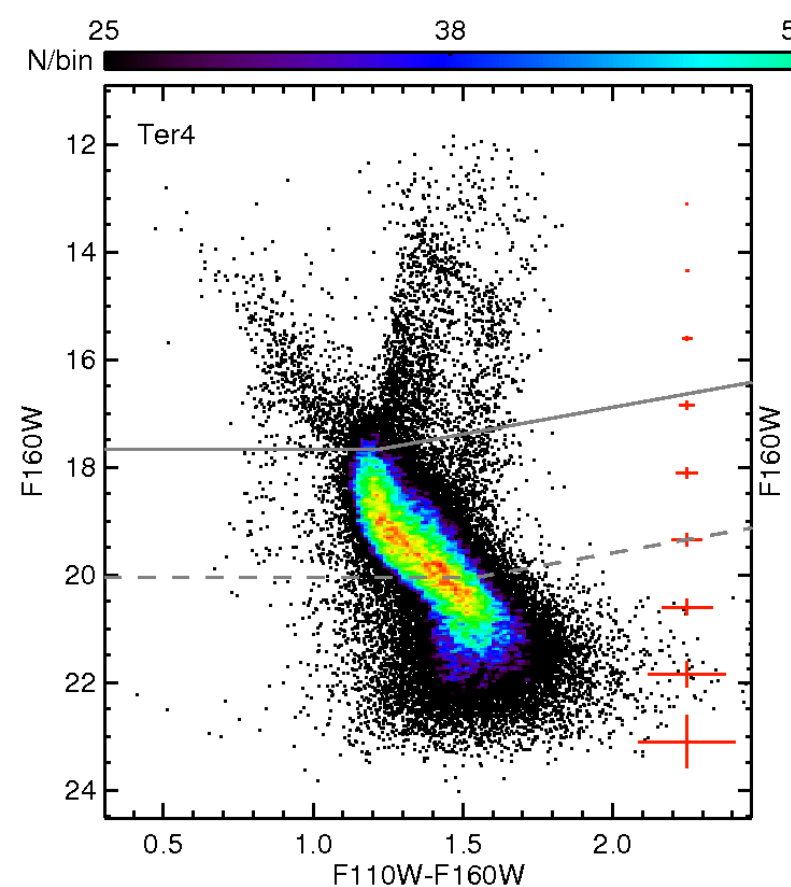

$58 \quad 88$

134
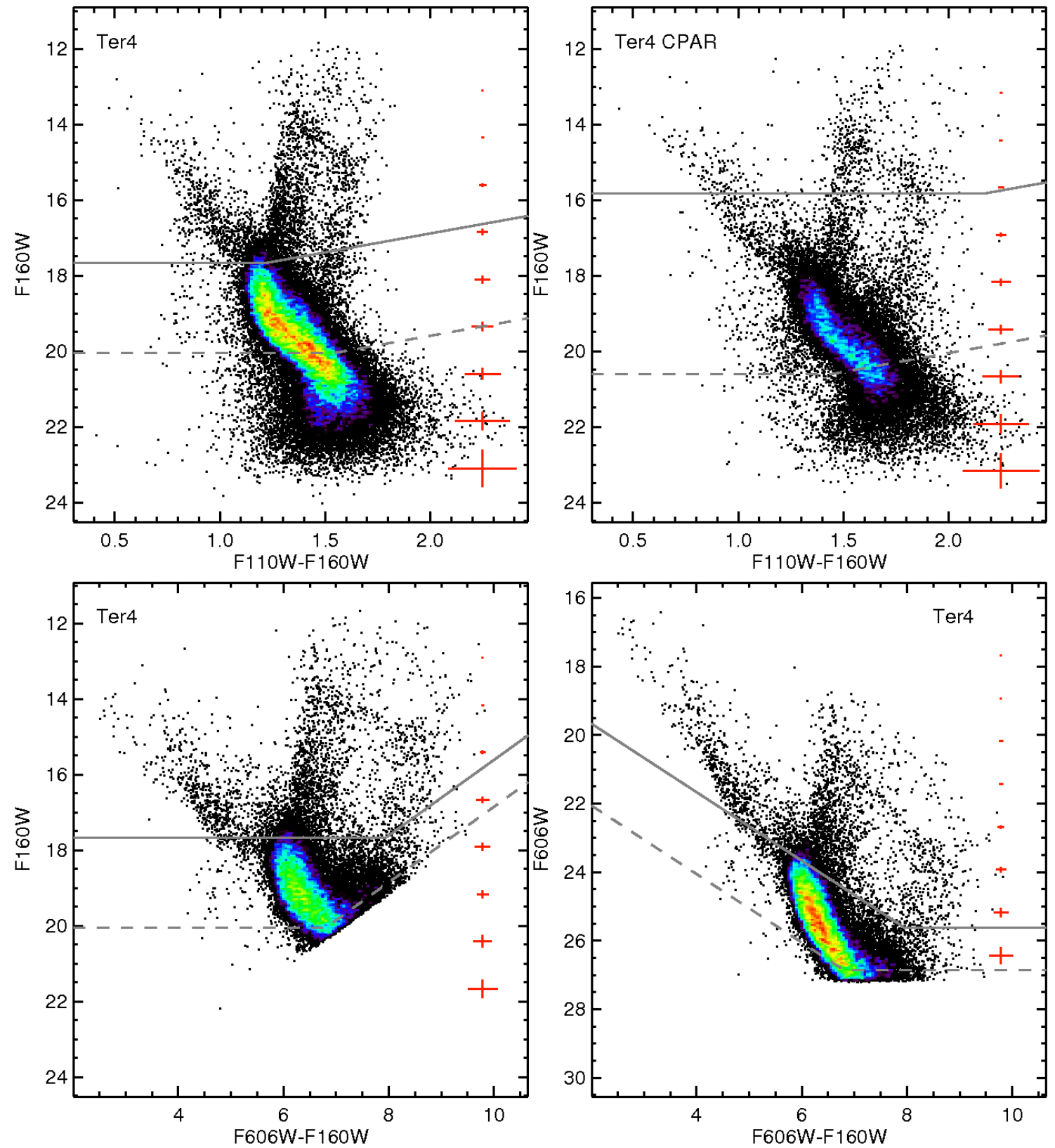

Figure 17. As in Fig. 7, but for Terzan 4.

Saracino, S., Dalessandro, E., Ferraro, F. R., et al. 2016, ApJ, 832, 48

Sarajedini, A., Bedin, L. R., Chaboyer, B., et al. 2007, AJ, 133,1658

Schlafly, E. F., Green, G. M., Lang, D., et al. 2018, ApJS, 234, 39

Siegel, M. H., Majewski, S. R., Law, D. R., et al. 2011, ApJ, 743, 20

Simunovic, M., \& Puzia, T. H. 2016, MNRAS, 462, 3401

Smith, H. A., \& Stryker, L. L. 1986, PASP, 98, 453
Stephens, A. W., \& Frogel, J. A. 2004, AJ, 127, 925

Tang, B., Cohen, R. E., Geisler, D., et al. 2017, MNRAS, 465,19

Trager, S. C., King, I. R., \& Djorgovski, S. 1995, AJ, 109, 218

Valencic, L. A., Smith, R. K., Dwek, E., Graessle, D., \& Dame, T. M. 2009, ApJ, 692, 502

Valenti, E., Ferraro, F. R., \& Origlia, L. 2004, MNRAS, 351,1204 


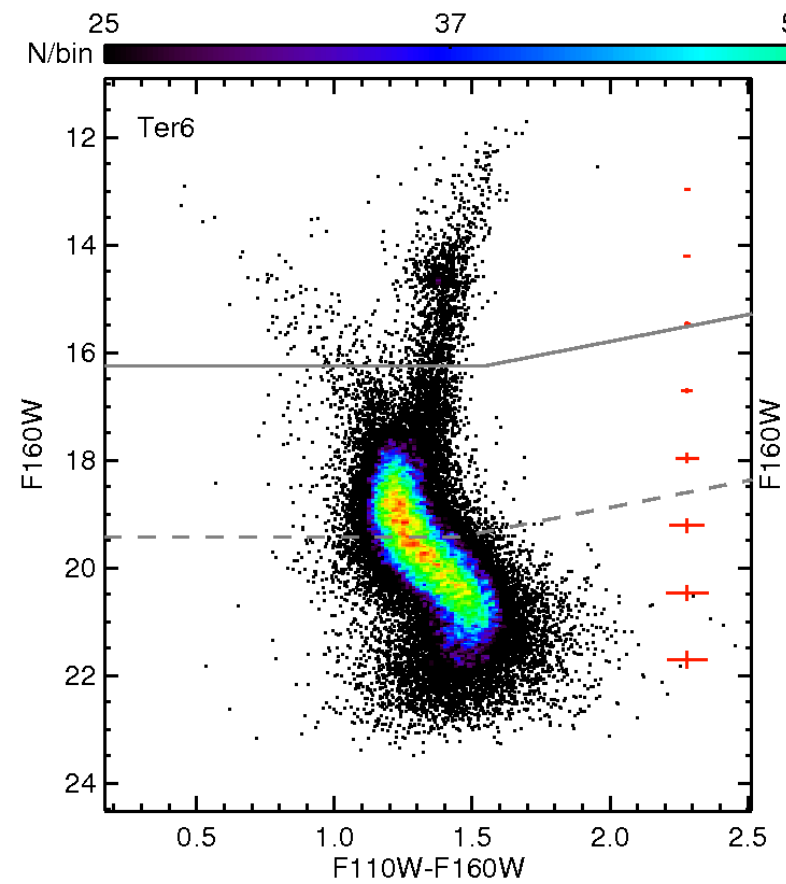

56

84
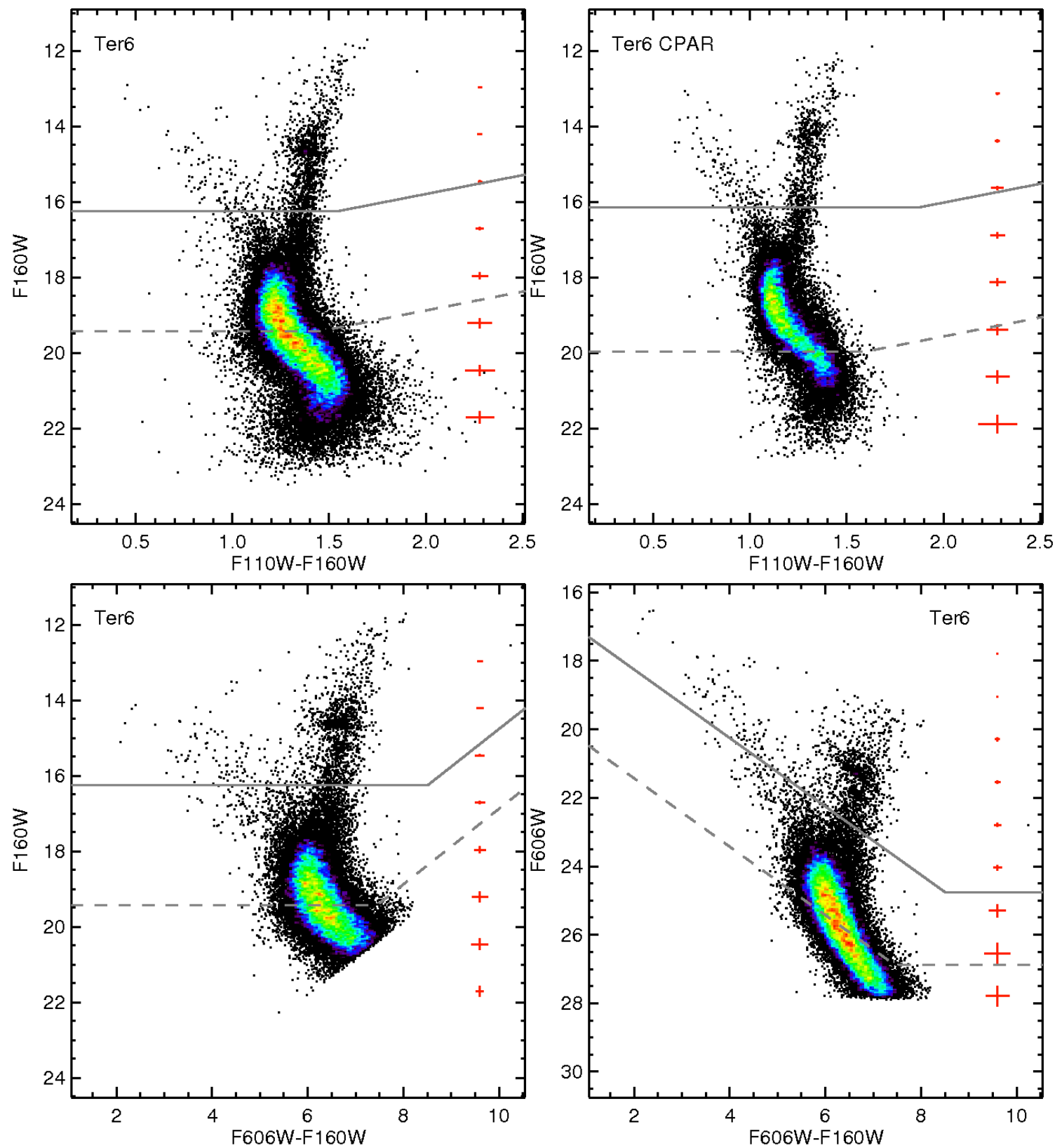

Figure 18. As in Fig. 7, but for Terzan 6.

Valenti, E., Origlia, L., \& Ferraro, F. R. 2005, MNRAS, 361,272

Valenti, E., Ferraro, F. R., \& Origlia, L. 2010, MNRAS, 402,1729

Valenti, E., Origlia, L., \& Rich, R. M. 2011, MNRAS, 414, 2690

Valenti, E., Origlia, L., Mucciarelli, A., \& Rich, R. M. 2015, A\&A, 574, A80

VandenBerg, D. A., Brogaard, K., Leaman, R., \& Casagrande, L. 2013, ApJ, 775, 134
Wagner-Kaiser, R., Stenning, D. C., Sarajedini, A., et al. 2016, MNRAS, in press

Wagner-Kaiser, R., Sarajedini, A., von Hippel, T., et al. 2017, arXiv:1702.08856

Watkins, L. L., van der Marel, R. P., Bellini, A., \& Anderson, J. 2015a, ApJ, 803, 29

Watkins, L. L., van der Marel, R. P., Bellini, A., \& Anderson, J. 2015b, ApJ, 812, 149

Wijnands, R., Heinke, C. O., \& Grindlay, J. E. 2002, ApJ, 572,1002 


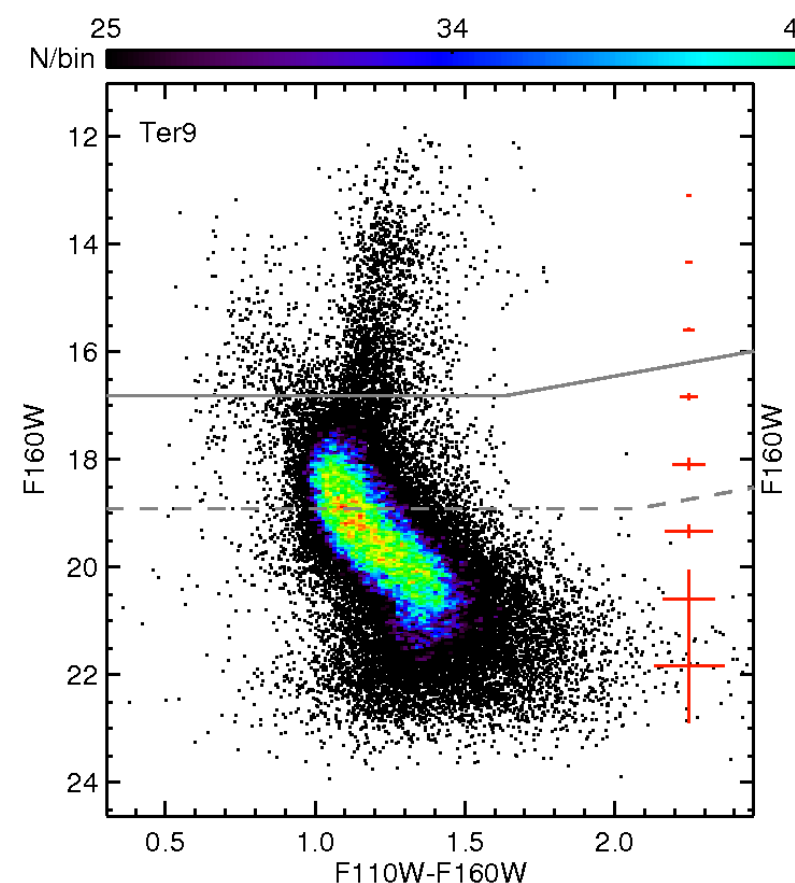

47

65
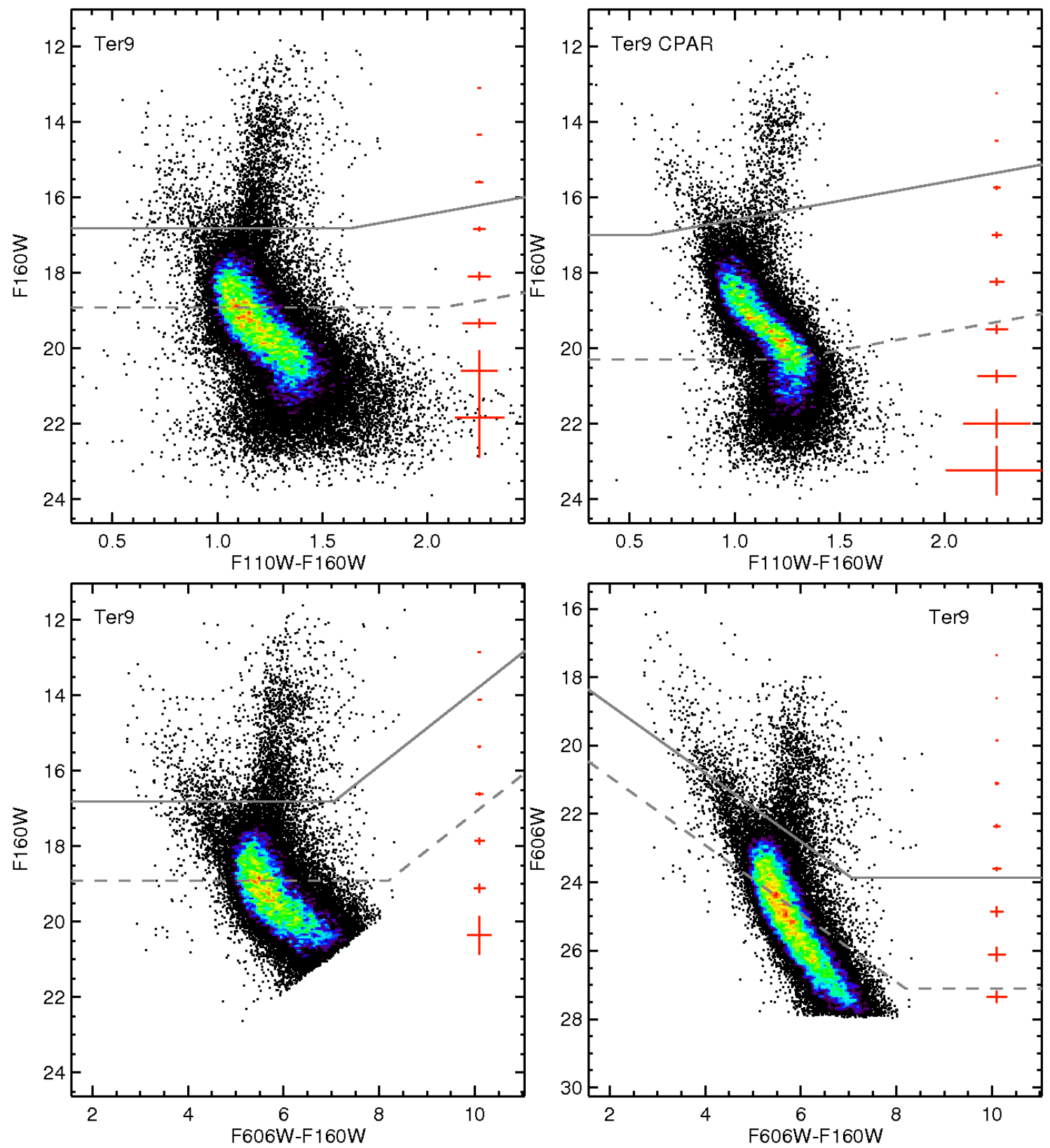

Figure 19. As in Fig. 7, but for Terzan 9.

Williams, B. F., Lang, D., Dalcanton, J. J., et al. 2014,

$$
\text { ApJS, 215, } 9
$$

Zinn, R., \& West, M. J. 1984, ApJS, 55, 45
Zoccali, M., Gonzalez, O. A., Vasquez, S., et al. 2014, A\&A, 562, A66

Zoccali, M., Vasquez, S., Gonzalez, O. A., et al. 2016, arXiv:1610.09174 


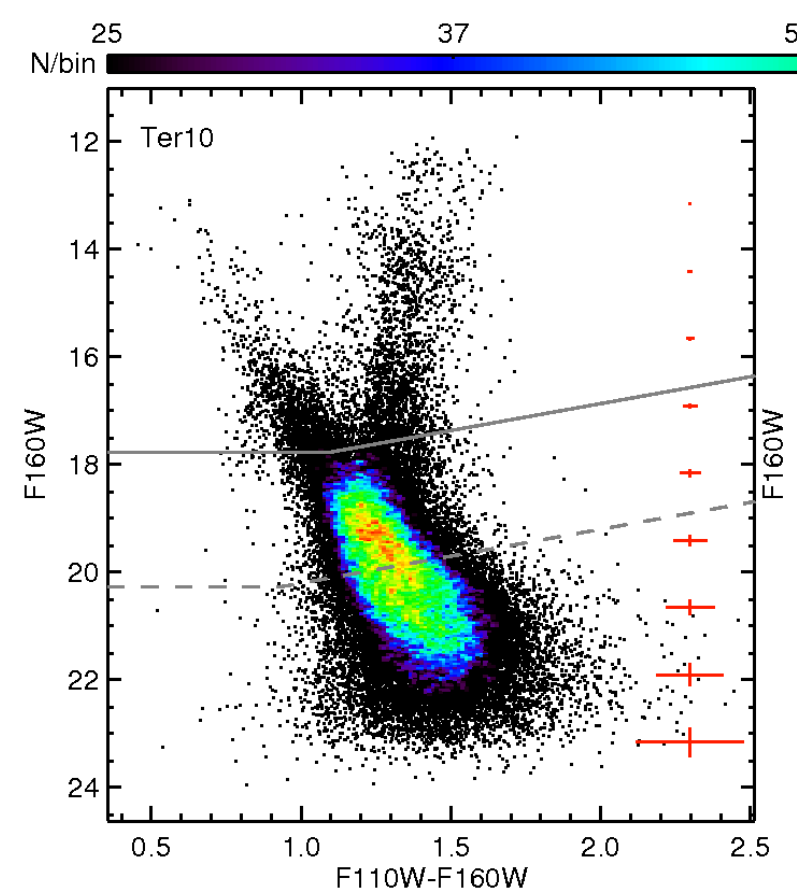

$55 \quad 81 \quad 119$
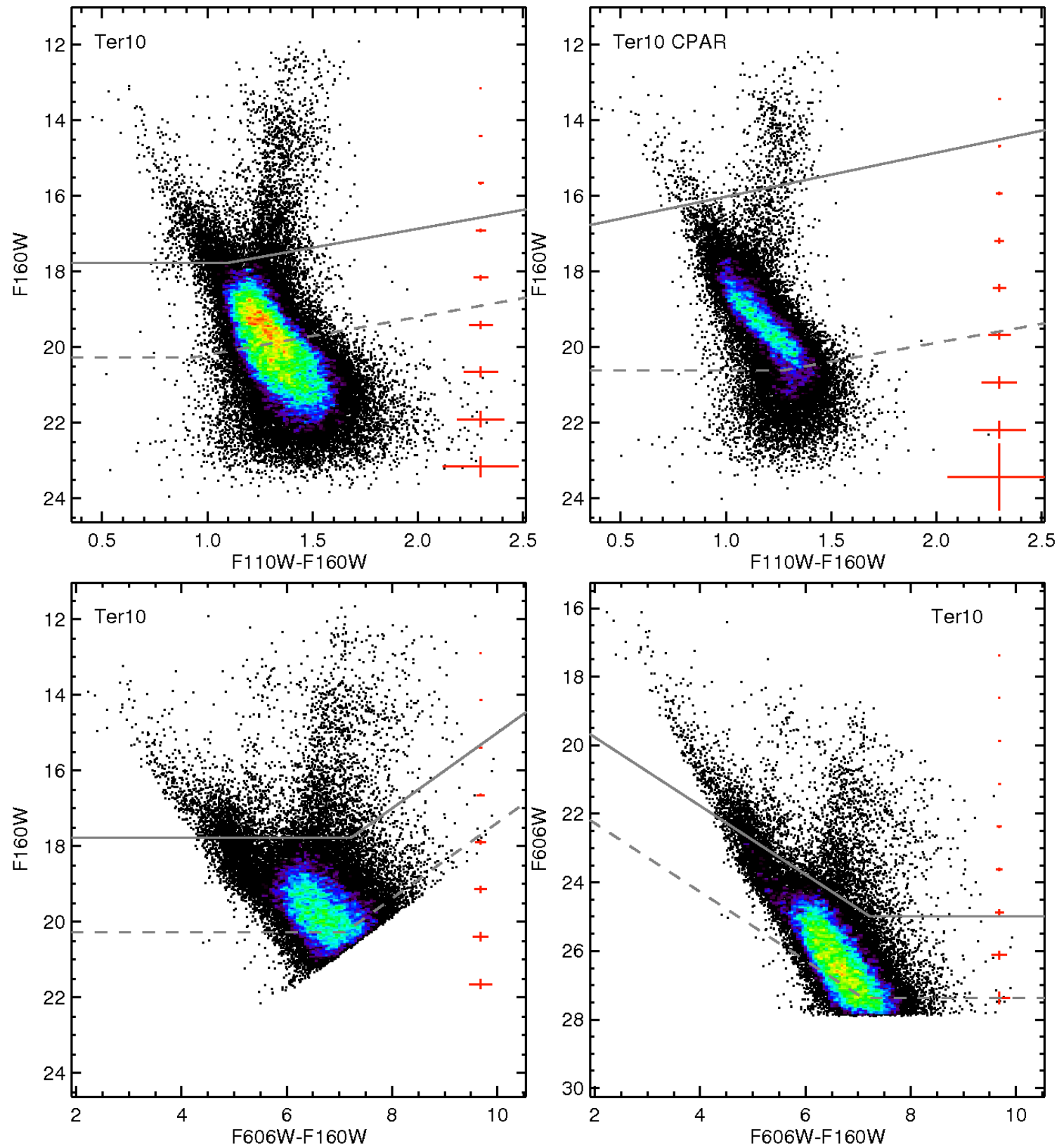

Figure 20. As in Fig. 7, but for Terzan 10 . 


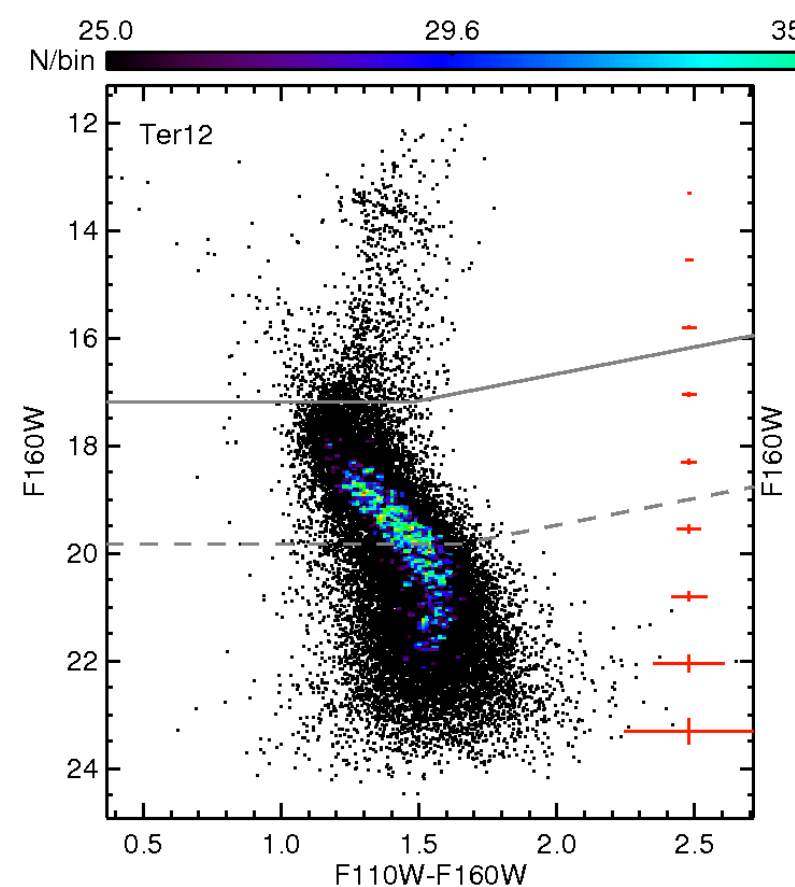

$35.0 \quad 41.4$

49.0
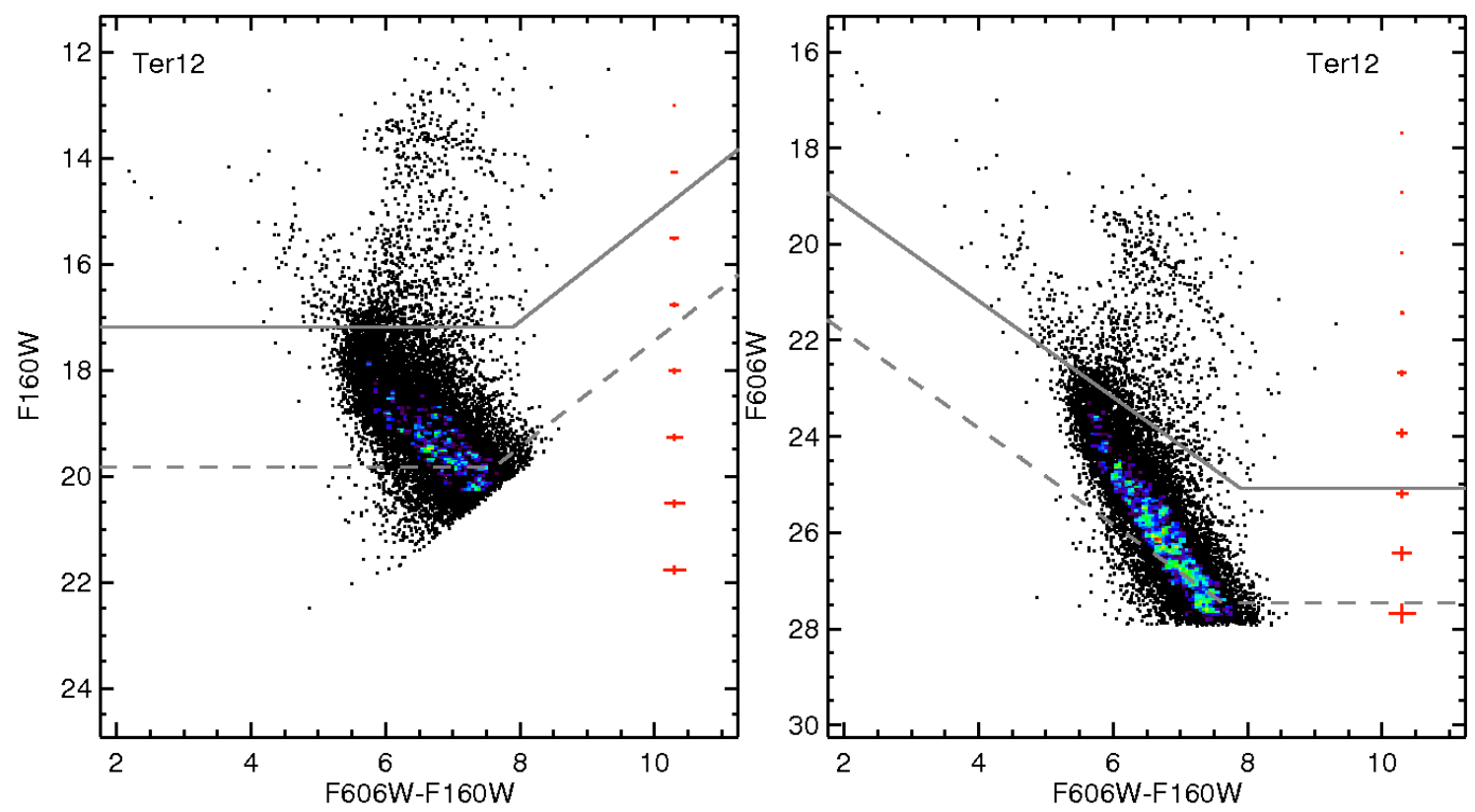

Figure 21. As in Fig. 7, but for Terzan 12 . 


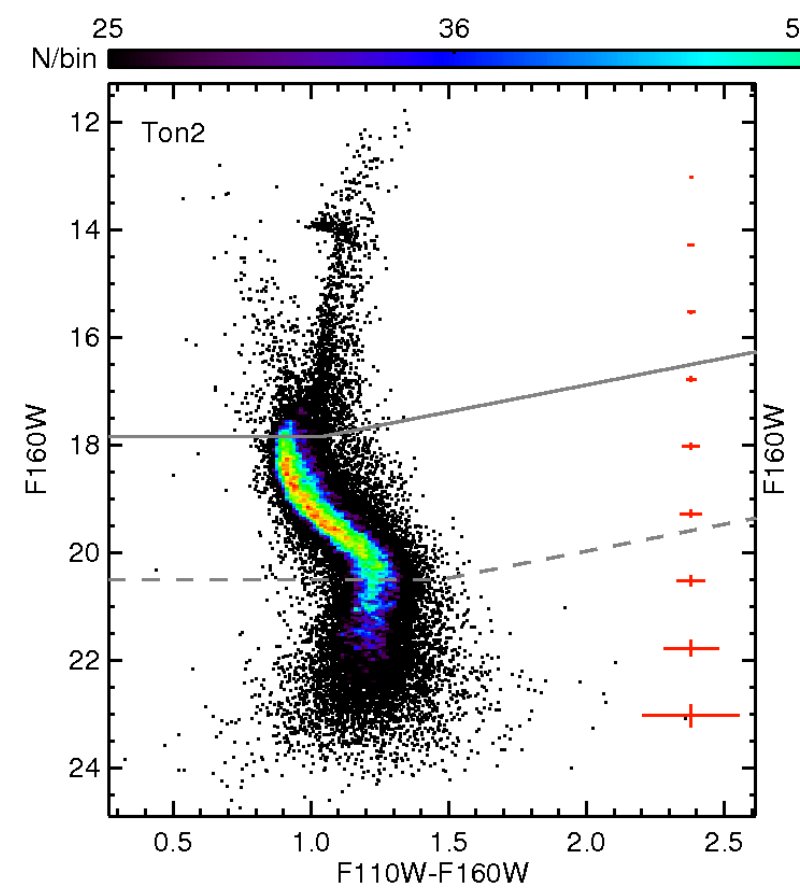

$53 \quad 77 \quad 112$
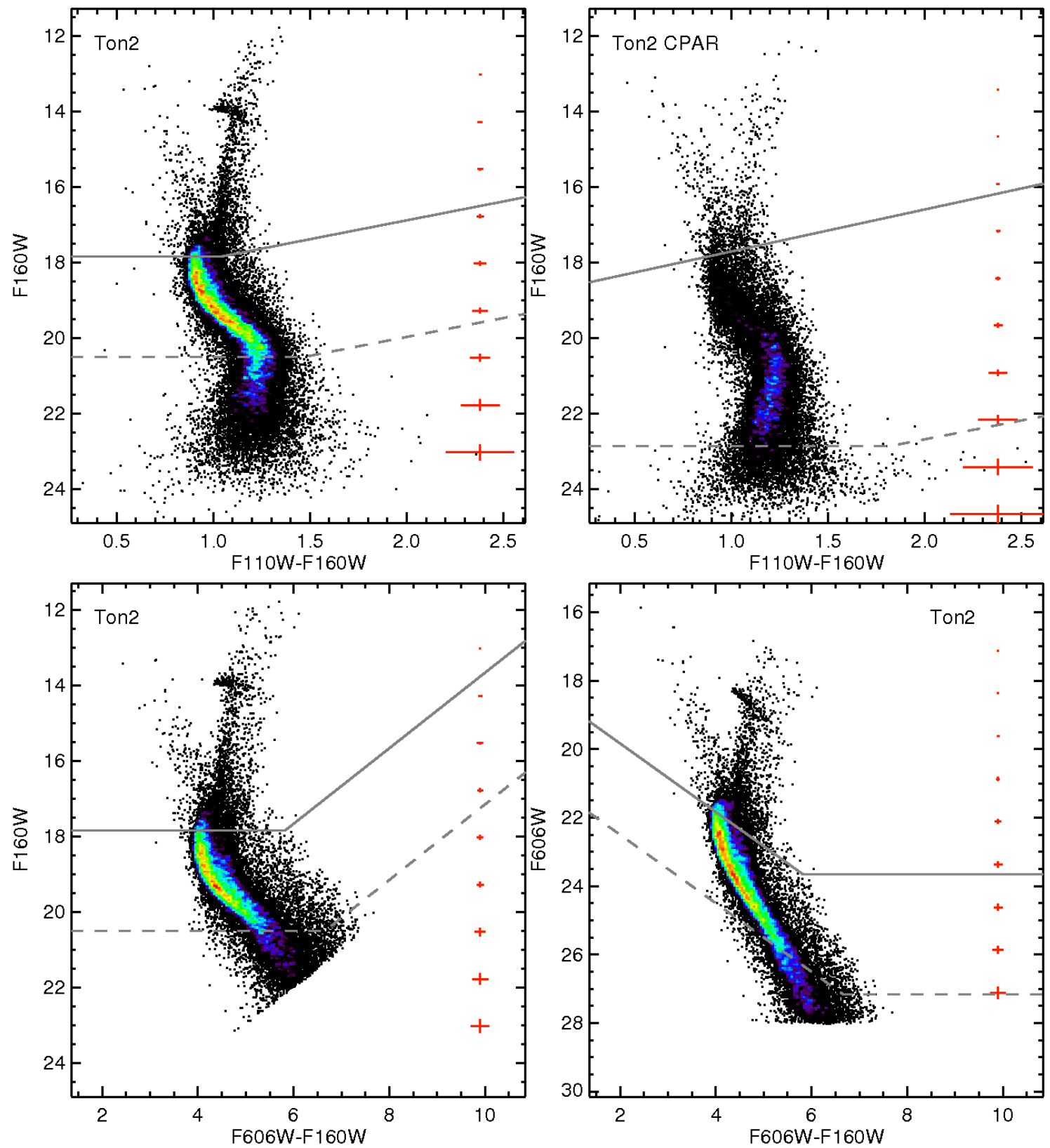

Figure 22. As in Fig. 7, but for Ton 2 . 